\title{
GROUND-WATER DATA FOR MICHIGAN
}

\author{
BY \\ G.C. HUFFMAN
}

U.S. GEOLOGICAL SURVEY

Open-File Report $80-1212$

PREPARED IN COOPERATION WITH THE MICHIGAN DEPARTMENT OF NATURAL RESOURCES GEOLOGICAL SURVEY DIVISION 


\section{CONVERSION FACTORS}

The following factors may be used to convert the English units published in this report to the International System of Units (SI).

\begin{tabular}{lccll} 
Multiply English units & & By & & To obtain SI units \\
\cline { 1 - 1 } acres (a) & & 0.4047 & & hectares (ha) \\
feet (ft) & .3048 & & meters (m) \\
inches (in) & 25.4 & & millimeters (mm) \\
miles (mi) & 1.609 & & kilometers (km) \\
million gallons (106 gal) & 3,785 & & cubic meters (m $\left.{ }^{3}\right)$ \\
gallons (gal) & 3.785 & & liters (1)
\end{tabular}


UNITED STATES

DEPARTMENT OF THE INTERIOR

GEOLOGICAL SURVEY

GROUND-WATER DATA FOR MICHIGAN 1979

by G. C. Huffman

U.S. GEOLOGICAL SURVEY

OPEN-FILE REPORT 80-1212

Prepared in cooperation with the Michigan

Department of Natura1 Resources

Lansing, Michigan

1980 
UNITED STATES DEPARTMENT OF THE INTERIOR

CECIL D. ANDRUS, Secretary

GEOLOGICAL SURVEY

H. William Menard, Director

For additional information write to:

Office of the District Chief

Water Resources Division

U.S. Geological Survey

6520 Mercantile Way, Suite 5

Lansing, Michigan 48910 
Introduction - 1

Purpose -

Scope -

Uses of data in this report .... 1

Availability of records -.... 3

Other ground-water reports

We11-numbering system -....... 9

Ground-water levels in 1979

Area ground-water data -

A1pena County -........ 12

Branch County - city of Coldwater -......- 13

Calhoun County - city of Battle Creek -...-.-...-.-14

Clinton County - city of St. Johns -.....- 15

Eaton County - Delta Township -...-.

Grand Traverse County -.......... 17

Ingham County - city of Lansing -

- city of Mason -.-.-.-.-19 19

- East Lansing-Meridian Water Authority -- 20

- Lansing Township -..--

Jackson County - Michigan State University - -

Kalamazoo County $\quad$ - city of Jackson

- city of Portage -.-.-1.- 25

Marquette County - Iron Range area -...-.-.-.-.-.-- 26

Oakland County - Huron-Clinton Metropolitan Authority --- 27

Sanilac County -........ 28

Van Buren County -....... 29

Washtenaw County - city of Ann Arbor -....... 30

- city of Ypsilanti -...-- 31

- Ypsilanti Township -...-_. 32

Tables -

Selected references - 49

Acknowledgments - 55 


\section{EIGURES}

Page

1. Map showing distribution of observation wells -............- 2

2. Map showing areas in the Upper Peninsula where ground-water conditions are described in published reports -............- 4

3. Map showing areas in the Lower Peninsula where ground-water conditions are described in published reports -...........- 6

4. Graphs of water levels in selected wells 11

\section{TABLES}

Page

1. Records of Michigan observation we11s

2. Reported ground-water pumpage

3. Water-quality data 
by

G. C. Huffman

INTRODUCTION

Purpose

The purpose of this report is to make available the 1979 records of water levels and related data for the principal aquifers of the State. This report is written for municipalities, industries, institutions, consultants, drillers, hydrologists, and other people interested in the ground-water resources.

\section{Scope}

Data on the yield of we1ls, pumpage, quality of water, and trends of ground-water levels for the past 5 years are shown in the text. Many hydrographs are included to illustrate natural changes in water levels. A1so included are data on municipal water-supply sources.

Tables 1, 2, and 3 contain records of water levels in observation wells, records of pumpage by most major ground-water users, and water quality data from selected wells sampled during 1979. Figure 1 shows distribution of observation wells.

\section{Uses of data in this report}

An effective method of determining the amount of water available from an aquifer is analysis of records of water levels and pumpage. Water-level records in pumped areas obtained by means of recorders indicate day-to-day and long-term effects of pumping. This information can be used by municipalities, industries, and institutions to estimate the capacity of aquifers to meet present and future demands for water and whether expansion of present supply systems for ground water is practicable. When new wells are to be installed in an area where water levels are declining because of pumping, projections of future water levels will indicate the depth below which the intake should be installed. Allowing for probable lowering of water levels, can extend the life of the installation. 


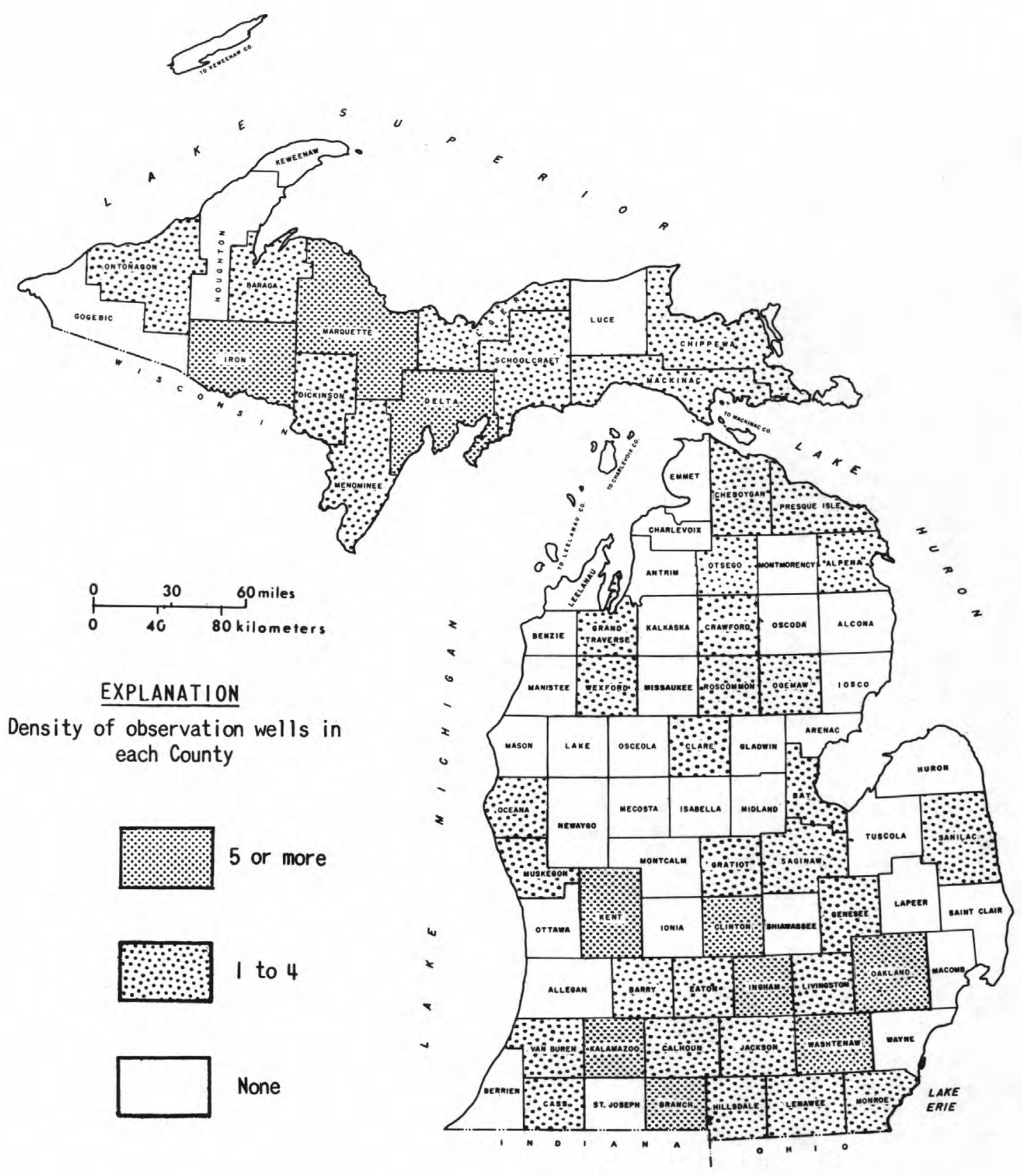

Figure 1.--Distribution of observation we11s. Water levels were monitored in 138 observation wells in 1979. 
The water table fluctuates an average of 2 to 3 feet $(0.6$ to $0.9 \mathrm{~m})$ annually and about 5 feet $(1.5 \mathrm{~m})$ over a period of years. Thus, if an excavation for a basement or septic tank is made when the water table is low, good construction practices would allow for the probable higher water levels in the spring. If construction is made after several years of drought, the allowance for the subsequent rise in water levels would be larger. If a site is at all questionable, the depth to the water table could be determined by boring and allowances made for the probable rise in water levels.

\section{Availability of records}

Complete tabulations of water-1evel measurements, hydrographs for observation wells, records of chemical quality, water-temperature measurements, well records and logs, aquifer tests, records of pumping for public and industrial supplies, and water-resources reports are on file for public inspection. They may be examined at the Geological Survey Division, Michigan Department of Natural Resources, Mason Building, Lansing, Michigan 48909; or at the U.S. Geological Survey, 6520 Mercantile Way, Suite 5, Lansing, Michigan 48910. Records for the Northern Peninsula are also kept on file in the State and Federal Geological Survey Offices, State Office Building, Escanaba, Michigan, 49829.

\section{Other ground-water reports}

From 1935 to 1974, records of ground-water levels in Michigan were published in U.S. Geological Survey Water-Supply Papers (WSP). Since 1975, these records have been published in U.S. Geological Survey Water-Data Reports (WDR).

To supplement the Water-Supply Paper and Water-Data Report series, publication of anmual reports, titled "Summary of Ground-Water Conditions in Michigan," was begun in 1956. Beginning in 1967, the title of the reports was changed to "Summary of Ground-Water Hydrological Data in Michigan," and in 1973 to "Ground-Water Data for Michigan."

Reports that describe ground water in Michigan are shown in figures 2 and 3. In addition, many publications dealing with ground water are 1isted in the selected references at the end of this report. 


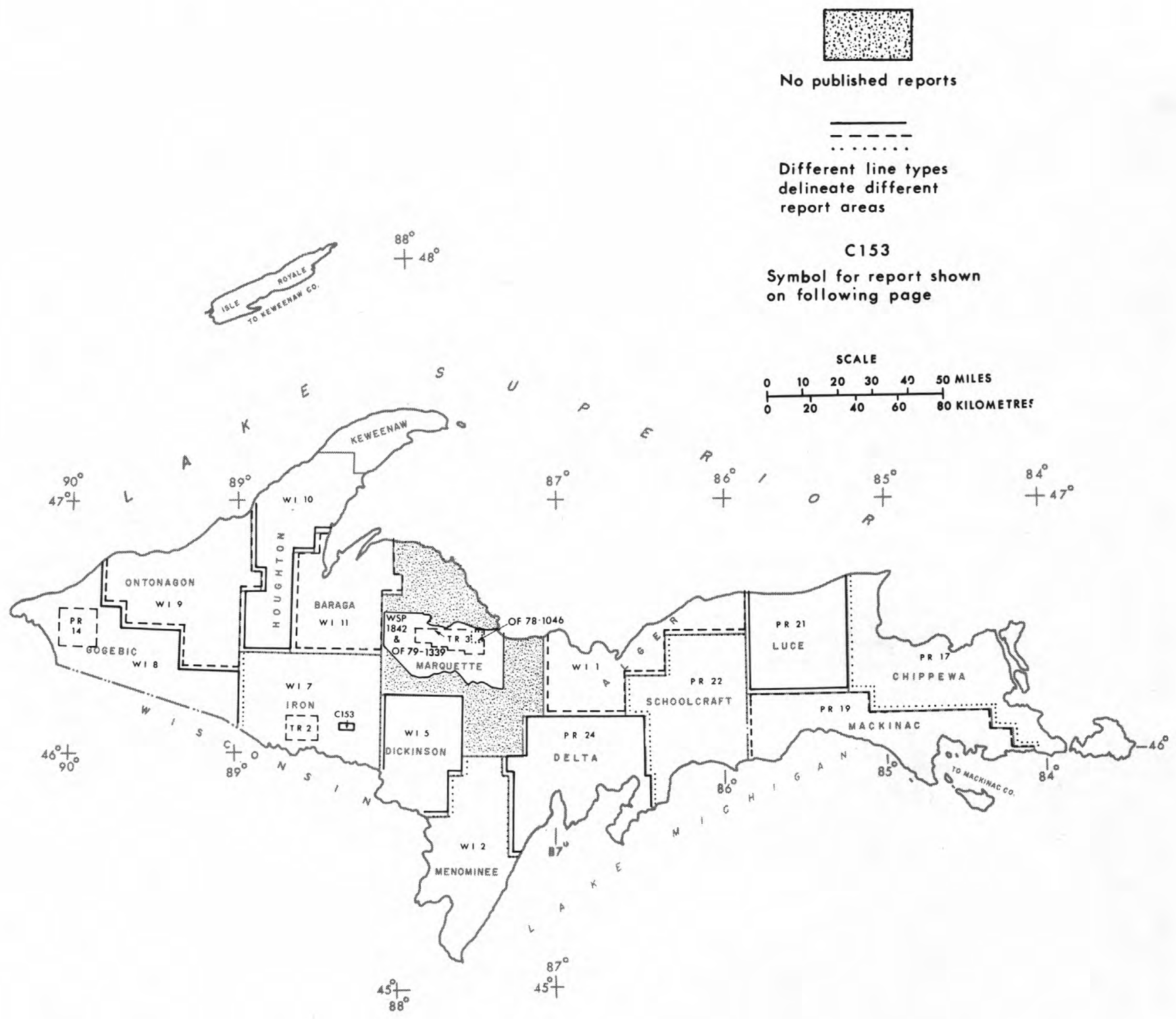

Figure 2.--Areas in the Upper Peninsula where ground-water conditions are described in published reports. 
C 153 -- Pettijohn, F. J., 1952, Geology of the northern Crystal Fal1s area, Iron County, Michigan: U.S. Geol. Survey Circ. 153.

\section{Open-File Reports}

OF 78-1046 -- Grannemann, N. G., 1978, Water supply potential of the Lake Sally system, Marquette County Michigan: U.S. Geologica1 Survey Open-File Report 78-1046.

OF 79-1339 -. 1979, Water resources of the Marquette Iron Range area, Marquette County, Michigan: U.S. Geological Survey Open-File Report 79-1339.

Professional Papers

Prof. Pap. 754A -- Huber, N. K., 1973, Glacial and postglacial geologic history of Isle Royale National Park, Michigan: U.S. Geol. Survey Prof. Paper 754-A.

\section{Progress Reports}

PR 14 -- Brown, E. A., and Stuart, W. T., 1951, Ground-water resources of the glacial deposits in the Bessemer area, Michigan: Michigan Geo1. Survey Prog. Rept. 14.

PR 17 -- Vanlier, K. E., and Deutsch, Morris, 1958, Reconnaissance of the ground-water resources of Chippewa County, Michigan: Michigan Geol. Survey Prog. Rept. 17.

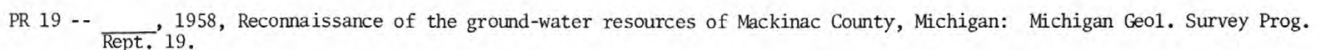

PR 21 -- Vanlier, K. E., 1959, Reconnaissance of the ground-water resources of Luce County, Michigan: Michigan Geol. Survey Prog. Rept. 21.

PR 22 -- Sinclair, W. C., 1959, Reconnaissance of the ground-water resources of Schoolcraft County, Michigan: Michigan Geol, Survey Prog. Rept. 22.

PR 24 -- Rept. 24. , Reconnaissance of the ground-water resources of Delta County, Michigan: Michigan Geol, Survey Prog.

\section{Technical Reports}

TR 2 -- Stuart, W. T., Theis, C. V., and Stanley, G. M., 1948, Ground-water problems in the Iron River district, Michigan: Michigan Geol, Survey Tech. Rept. 2.

TR 3 -- Stuart, W. T., Brown, E. A., and Rhodehame1, E. C., 1954, Ground-water investigations of the Marquette iron-mining district, Michigan: Michigan Geol. Survey Tech. Rept. 3.

\section{Water Investigations}

WI 1 -- Vanlier, K. E., 1963, Ground water in Alger County: Michigan Geol. Survey Water Inv. 1.

WI 2 -, 1963, Ground water in Menominee County: Michigan Geol. Survey Water Inv. 2.

WI 5 -- Hendrickson, G. E., and Doonan, C. J., 1966, Ground-water resources of Dickinson County, Michigan: Michigan Geol. Survey Water Inv. 5.

WI 7 -- Doonan, C. J., Hendrickson, G. E., 1967, Ground water in Iron County, Michigan: Michigan Geol. Survey Water Inv. 7.

WI 8 -, 1968, Ground water in Gogebic County, Michigan: Michigan Geo1. Survey Water Inv. 8.

WI 9 -, 1969, Ground water in Ontonagon County, Michigan: Michigan Geol. Survey Water Inv. 9.

WI 10 -- Doonan, C. J., Hendrickson, G. E., and Byerlay, J. R., 1970, Ground water and geology of Keweenaw Peninsula, Michigan: Michigan Geol. Survey Water Inv. 10

WI 11 -- Doonan, C. J., and Byerlay, J. R., 1973, Ground water and geology of Baraga County, Michigan: Michigan Geol. Survey Water Inv. 11

\section{Water-Supply Papers}

WSP 1842 -- Wiitala, S. W., Newport, T. G., and Skinner, E. L., 1967, Water resources of the Marquette Iron Range area, Michigan: U.S. Geol. Survey Water-Supply Paper 1842. 


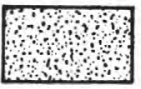

No published reports

$\rightarrow---$

Different line types delineate different report areas

\section{C183}

Symbol for report shown on following page

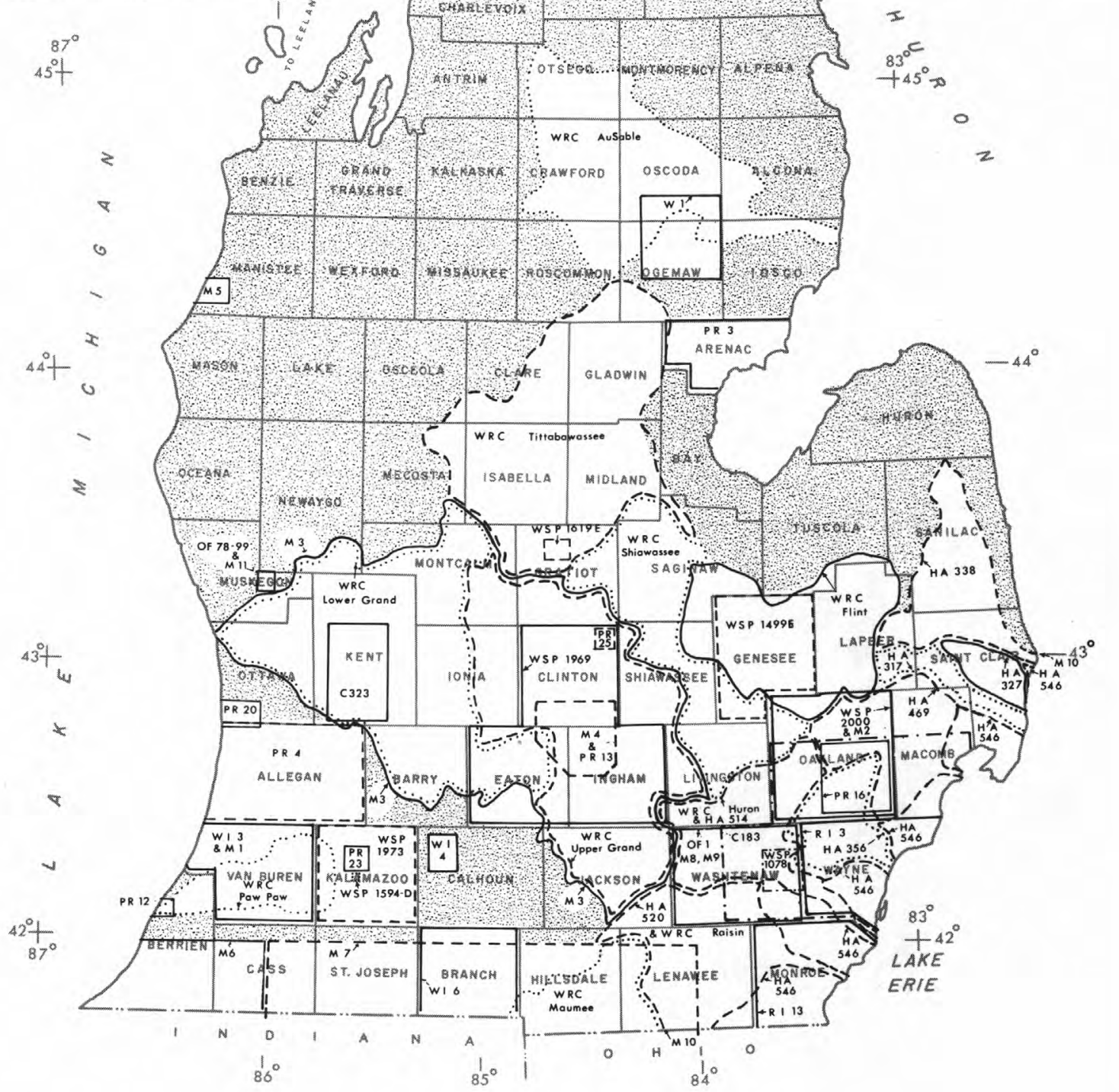

Figure 3.--Areas in the Lower Peninsula where ground-water conditions are described in published reports. 
C 183 -- Wisler, C. 0., Strame1, G. J., and Laird, L. B., 1952, water resources of the Detroit area, Michigan: U.S. Geol. Survey Circ. 183 .

C 323 -- Stramel, G. J., Wisler, C. O., and Laird, L. B., 1954, Water resources of the Grand Rapids area, Michigan: U.S. Geol. Survey Circ. 323

\section{Hydrologic Atlases}

HA 317 -- Knutilla, R. L., 1969, Water resources of the Belle River basin, southeastern Michigan: U.S. Geol. Survey Hydrol. Inv. At1as HA-317.

HA 327 -, 1969, Water resources of the Pine River basin, southeastern Michigan: U.S. Geo1. Survey Hydrol. Inv. At1as HA-327.

HA 338 -, 1970, Water resources of the Black River basin, southeastern Michigan: U.S. Geol, Survey Hydrol. Inv. Atlas HA-338.

HA 356 -, 1971, Water resources of the River Rouge basin, southeastern Michigan: U.S. Geo1. Survey Hydrol. Inv. At1as HA-356.

HA 469 -- Nowlin, J. O., 1973, Water resources of the Clinton River basin, southeastern Michigan: U.S. Geol. Survey Hydrol. Inv. Atlas HA- 469 .

HA 514 -- Larson, R. W., Allen, W. B., and Hanson, S. D., 1975, Water resources of the Huron River basin, southeastern Michigan: U.S. Geol. Survey Hydrol. Inv. Atlas HA-514.

HA 520 -- Knutilla, R. L., and Allen, W. B., 1975, water resources of the River Raisin basin, southeastern Michigan: U.S. Geol. Survey Hydrol. Inv. Atlas HA-520.

HA 546 -- Twenter, F. R., Knutilla, R. L., Cummings, T. R., 1975, Water resources of basins for minor streams draining into St. Clair River, Lake St. Clair, Detroit River, and Lake Erie, southeastern Michigan: U.S. Geo1. Survey Hydrol. Inv. Atlas HA-546.

\section{Misce11aneous Reports}

M 1 -- Terwilliger, F. W., 1954, The glacial geology and ground-water resources of Van Buren County, Michigan, pt. 1 of Occasional papers for 1954 on the geology of Michigan: Michigan Geol. Survey Pub. 48.

M 2 -- Mozola, A. J., 1954, A survey of ground-water resources in Oakland County, Michigan, pt. 2 of Occasional papers for 1954 on the geology of Michigan: Michigan Geol. Survey Pub. 48.

M 3 -- Vanlier, K. E., 1968, Appendix E of the report on the Grand River Comprehensive Basin Study: U.S. Army Eng. District, Detroit, Michigan.

M 4 -- Vanlier, K. E., and Wheeler, M. L., 1968, Analog simulation of ground-water development of the Saginaw Formation, Lansing metropolitan area, Michigan: Tri-County Planning Commission, Lansing Ground-Water Rept.

M 5 -- Childs, K. E., 1970, History of the salt, brine, and paper industries and their probable effect on the ground-water quality in the Manistee Lake area, Michigan: Michigan Dept. Nat. Resources.

M 6 -- Schneider, A. F., and Keller, S. J., 1970, Indiana Geological Survey regional geological map number 4: Indiana Dept. Nat. Resources.

M 7 -- Johnson, G. H., and Keller, S. J., 1972, Indiana Geological Survey regional geological map number 8: Indiana Dept. Nat. Resources.

M 8 -- Twenter, F. R., Knutil1a, R. L., and Nowlin, J. 0., 1976, Water resources of Washtenaw County, Michigan: Washtenaw County Metropolitan Plan. Comm.

M 9 -- Borton, T. E., 1974, Planning perspectives on water resources, Washtenaw County, Michigan: Washtenaw County Metropolitan Plan. Comm.

M 10 -- Twenter, F. R., 1975, Ground water and geology -- southeastern Michigan: U.S. Army Corps of Engineers.

M 11 -- Fleck, W. B., and McDonald, M. G., 1978, Three-dimensional finite-difference model of ground-water system underlying the Muskegon County wastewater disposal system, Michigan: U.S. Geol. Survey Jour. of Research, v. 6, no, 3 .

\section{Open-File Reports}

OF 1 - Fleck, W. B., 1974, Geology and hydrology for environmental planning in Washtenaw County, Michigan: U.S. Geol. Survey Open-File Report unnumbered.

OF 78-99 -- McDonald, M. G., and Fleck, W. B., 1978, Model analysis of the impact on ground-water conditions of the Muskegon County wastewater disposal system, Michigan: U.S. Geol. Survey Open-File Report 78-99.

\section{Progress Reports}

PR 3 -- Pringle, G. H., 1937, Geology of Arenac County, Michigan: Michigan Geol. Survey Prog. Rept. 3.

PR 4 -- Riggs, C. H., 1938, Geology of Allegan County, Michigan: Michigan Geo1. Survey Prog. Rept. 4.

PR 12 -- Stuart, W. T., and Stal1man, R. W., 1945, Ground-water resources of the Benton Harbor area, Michigan: Michigan Geol. Survey Prog. Rept, 12 


\section{Progress Reports--Continued}

PR 13 -- Stuart, W. T., 1945, Ground-water resources of the Lansing area, Michigan: Michigan Geol. Survey Prog. Rept. 13.

PR 16 -- Ferris, J. G., and others, 1954, Ground-water resources of southeastem Oakland County, Michigan: Michigan Geol. Survey Prog. Rept. 16

PR 20 -- Deutsch, Morris, Burt, E. M., and Vanlier, K. E., 1958, Summary of ground-water investigations in the Holland area, Michigan: Michigan Geol. Survey Prog. Rept. 20.

PR 23 -- Deutsch, Morris, Van1ier, K. E., and Giroux, P. R., 1960, Ground-water hydrology and glacia1 geology of the Kalamazoo area, Michigan: Michigan Geol. Survey Prog. Rept. 23. PR 25 -- Vanlier, K. E., 1962, Summary of ground-water investigations in the Elsie area, Michigan: Michigan Geol. Survey Prog.

\section{Reports of Investigations}

RI 3 -- Mozola, A. J., 1969, Geology for land and ground-water development in Wayne County, Michigan: Michigan Geol. Survey Rept. Inv. 3 RI 13 -. 1970, Geology for environmental planning in Monroe County, Michigan: Michigan Geol. Survey Rept. Inv, 13.

\section{Water Information Series}

W 1 -- Knutilla, R. L., Twenter, F. R., and Larson, R. W., 1971, Upper Rifle River Basin -- An Evaluation of its Water Resources and Hydrologic Environment: Michigan Geol. Survey Water Information Series Rept. 1.

\section{Water Investigations}

WI 3 -- Giroux, P. R., Hendrickson, G. E., Stoimenoff, L. E., and Whetstone, G. W., 1964, Water resources of Van Buren County, Michigan: Michigan Geo1. Survey Water Inv. 3.

WI 4 -- Vanlier, K, E., 1966, Ground-water resources of the Battle Creek area, Michigan; Michigan Geol. Survey Water Inv. 4.

WI 6 -- Giroux, P. R., Stoimenoff, L. E., Nowlin, J. O., and Skinner, E. L., 1966, Water resources of Branch County, Michigan: Michigan Geol. Survey Water Inv. 6.

\section{Michigan Water Resources Commission Reports}

WRC Au Sable -- Water resource conditions and uses in the Au Sable River Basin, 1966: Michigan Water Resources Comm. Rept.

WRC Flint -- Water resource conditions and uses in the Flint River Basin, 1956: Michigan Water Resources Corm. Rept.

WRC Huron -- Water resource conditions and uses in the Huron River Basin, 1957: Michigan Water Resources Corm. Rept.

WRC Lower Grand -- Water resource conditions and uses in the Lower Grand River Basin, 1967, (open file): Michigan Water Resources Comm. Rept.

WRC Maumee -- Water resource conditions and uses in the Maumee River Basin, 1964: Michigan Water Resources Corm. Rept.

WRC Paw Paw -- Water resource conditions and uses in the Paw Paw River Basin, 1955, (revised report in 1964): Michigan Water Resources Comm. Rept.

WRC Raisin -- Water resource conditions and uses in the River Raisin Basin, 1965: Michigan Water Resources Comm. Rept.

WRC Shiawassee -- Water resource conditions and uses in the Shiawassee River Basin, 1963: Michigan Water Resources Comm. Rept.

WRC Tittibawassee -- Water resource conditions and uses in the Tittibawassee River Basin, 1960: Michigan Water Resources Comm. Rept.

WRC Upper Grand -- Water resource conditions and uses in the Upper Grand River Basin, 1961: Michigan Water Resources Corm. Rept.

\section{Water Supply Papers}

WSP 1078 -- McGuinness, C. L., Poindexter, O. F., and Otten, E. G., 1949, Ground-water supplies of the Ypsilanti area, Michigan: U.S. Geol. Survey Water-Supply Paper 1078

WSP 1499E -- Wiitala, S. W., Vanlier, K. E., and Krieger, R. A., 1963, Water resources of the Flint area, Michigan: U.S. Geol. Survey Water-Supply Paper 1499-E.

WSP 15941) -- Reed, J. E., Deutsch, Morris, and Wiitala, S. W., 1966, Induced recharge of an artesian glacial-drift aquifer at Kalamazoo, Michigan: U.S. Geo1. Survey Water-Supply Paper 1594-D.

WSP 1619E -- Vanlier, K. E., 1963, Ground-water resources of the A1ma area, Michigan: U.S. Geol. Survey Water-Supply Paper 1619-E.

WSP 1969 -- Vanlier, K. E., Wood, W. W., and Brunett, J. 0., 1973, Water-supply development and management alternatives for C1inton, Eaton, and Ingham Counties, Michigan: U.S. Geol. Survey Water-Supply Paper 1969.

WSP 1973 -- Allen, W. B., Miller, J. B., and Wood, W. W., 1972, Availability of water in Kalamazoo County, Michigan: U.S. Geol. Survey Water-Supply Paper 1973.

WSP 2000 -- Twenter, F. R., and Knutilla, R. L., 1972, water for a rapidly growing urban community -- 0akland County, Michigan: U.S. Geol. Survey Water-Supply Paper 2000 


\section{We11-numbering system}

The well-numbering system for Michigan indicates the location of wells within the rectangular subdivision of the land with reference to the Michigan meridian and base line. The first two segments of the well number designate township and range, the third segment of the number designates the section and the letters A through D designate successively smaller subdivisions of the section, as shown below. Thus, a well designated as 32N 6E 16CCCB would be located to the nearest 2.5 acres ( 1 hectare) and would be within the shaded area in section 16.

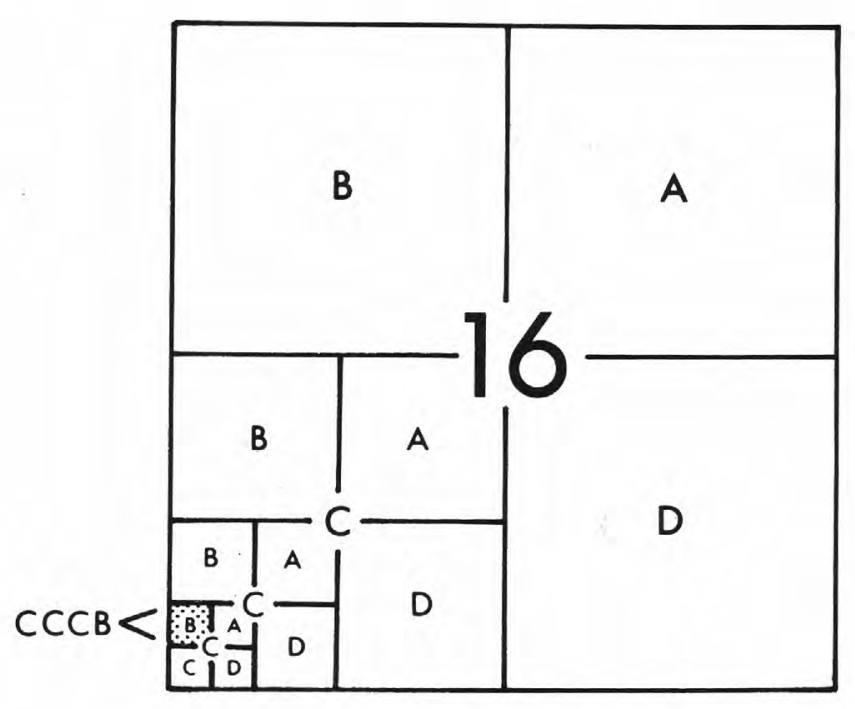

For many wells in this report, locations are only given to the nearest 40-acre (16 hectares) tract, for example, 16CC. In the event that two or more wells are in the same tract, a sequential number designation is added--for example, 16CCCB1, 16CCCB2, etc. The Michigan Geologica1 Survey uses a similar system except that numbers are used instead of letters. 
Water levels were measured in 138 observation we11s throughout the State (fig. 1 and table 1). In most areas, ground-water levels followed precipitation trends. In areas where precipitation was above normal, such as in the northern part of the State (fig. 4), ground-water levels were generally above average. Where precipitation was below normal, levels were generally below average. Although the quantity of precipitation is one of the major factors affecting ground-water levels, many other factors, such as soil conditions; time, duration, and intensity of precipitation; nature of underlying rocks; and slope of the land surface affect the levels.

Hydrographs of fluctuations of water levels in we11s (fig. 5) show that levels are highest in the spring. At this time, snowmelt and rain normally result in large additions to ground-water reservoirs. However, ice cover or frost in the ground can impede infiltration. Under these conditions, most water from snowmelt and precipitation runs off rapidly, and little goes to recharge the ground-water reservoirs. Recharge is small during the growing season, as most rainfall is evaporated, is transpired by vegetation, or runs off overland after heavy showers. In the fall, evapotranspiration is reduced by cold weather. At this time, heavy rains may cause water levels to rise. Frozen ground impedes the infiltration of water during the winter.

In addition to changes in water levels from precipitation, temporary changes in levels may be caused by earth tides and variation in barometric pressure. Evapotranspiration causes small daily declines in water levels in some we11s.

\section{AREA GROUND-WATER DATA}

Descriptions of some of Michigan's municipal, institutional, and areal ground-water supplies follow alphabetically, by counties. Yield of wells and pumpage data are given as they were reported by water departments and consultants. Included are data on the chemical quality of water, based on latest analyses made by the Michigan Department of Health. Where more than one we11 is involved, a range in quality is generally given. In this report, the unit milligrams per liter (mg/L) can be considered to be numerically equal to parts per million (ppm). Most descriptions are supplemented by illustrations. 


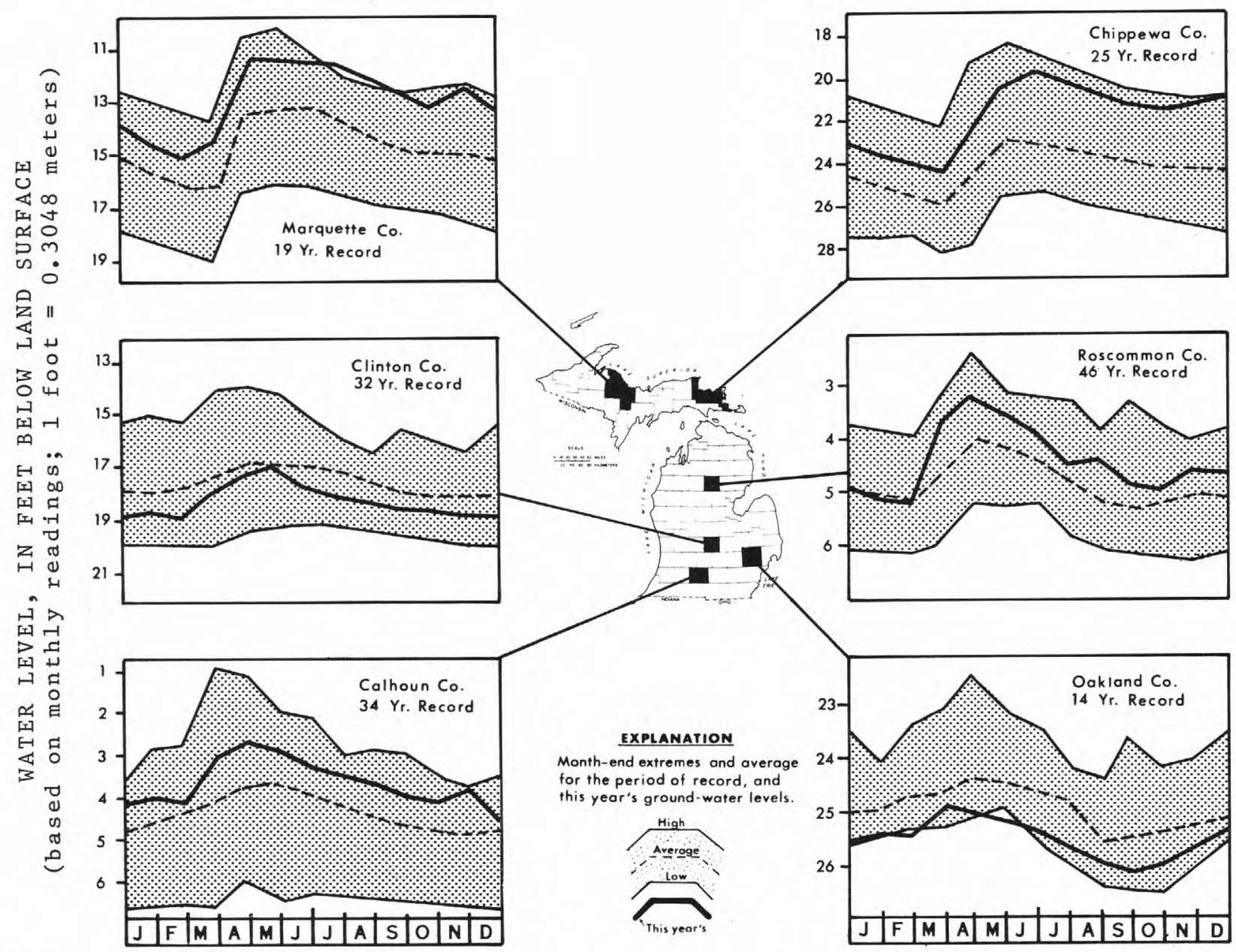

Figure 4.--Water 1evels in selected we1ls during 1979. 

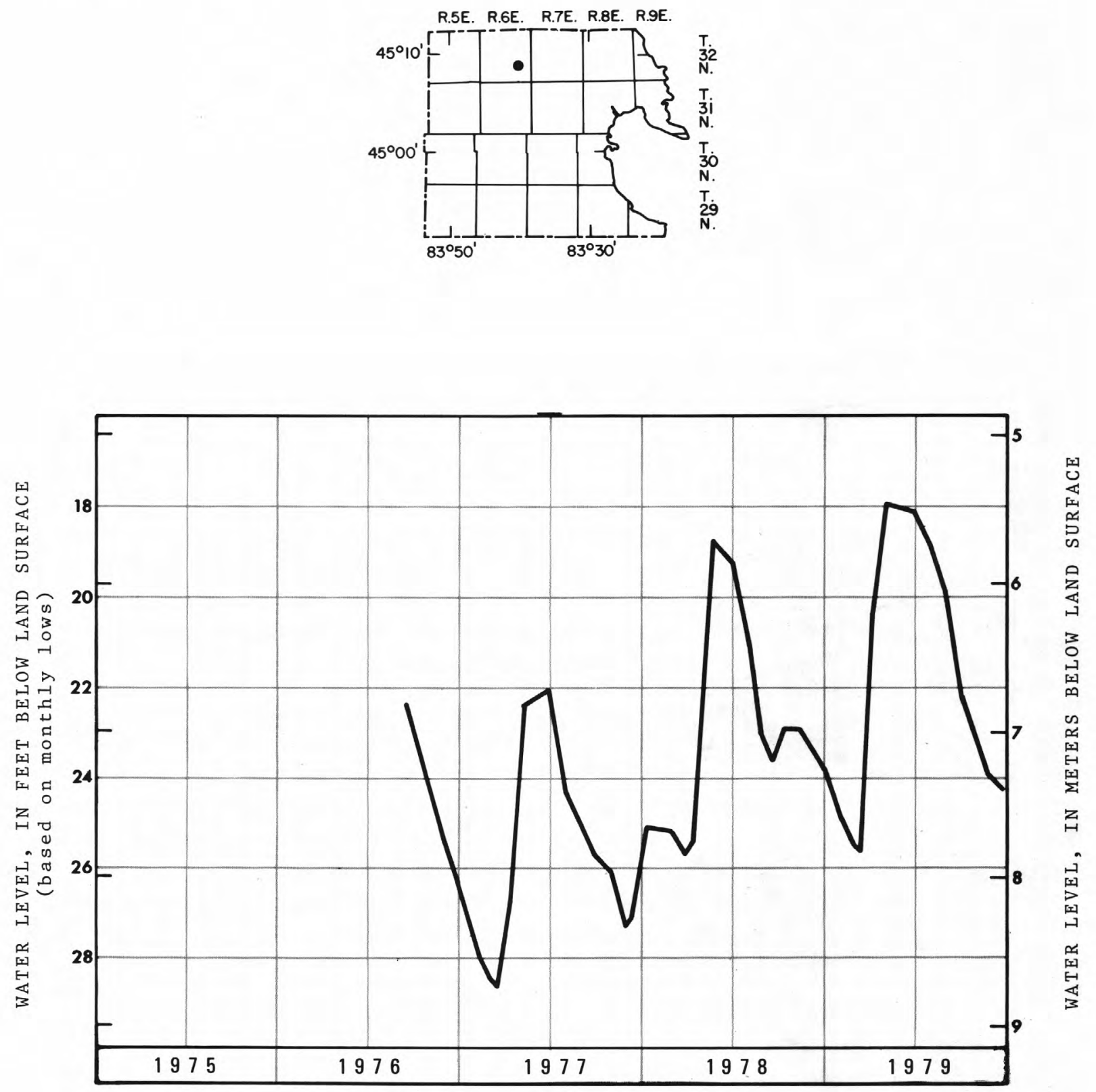

Geologica1 Survey observation we11 32N 6E 23DDDA1. We11 is 88 feet deep and in sand. Water-quality data in previous ground-water report (Huffman, 1979). 
SUPPLY AND SOURCE - - 4 we11s, 117 to 129 feet deep, tap glacial deposits. YIELD OF WELLS -- No. 3 - 1,200; No. 4 - 1,400; No. 5 - 2,250; No. 6 $2,850 \mathrm{gal} / \mathrm{min} ;$ specific capacity -- 80 to $190 \mathrm{ga} 1 / \mathrm{min} / \mathrm{ft}$ of $\mathrm{drawdown}$. PUMPAGE -- Tota1 annual pumpage, in million gallons, for past 5 years.

$$
\begin{array}{r}
1979-1,209 \\
1978-1,223 \\
1977-1,154 \\
1976-1,085 \\
1975-965
\end{array}
$$

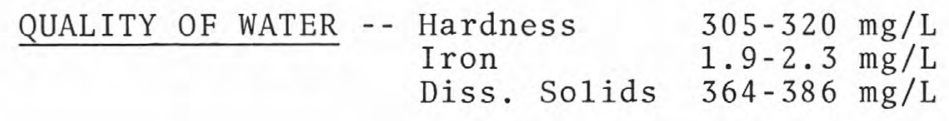

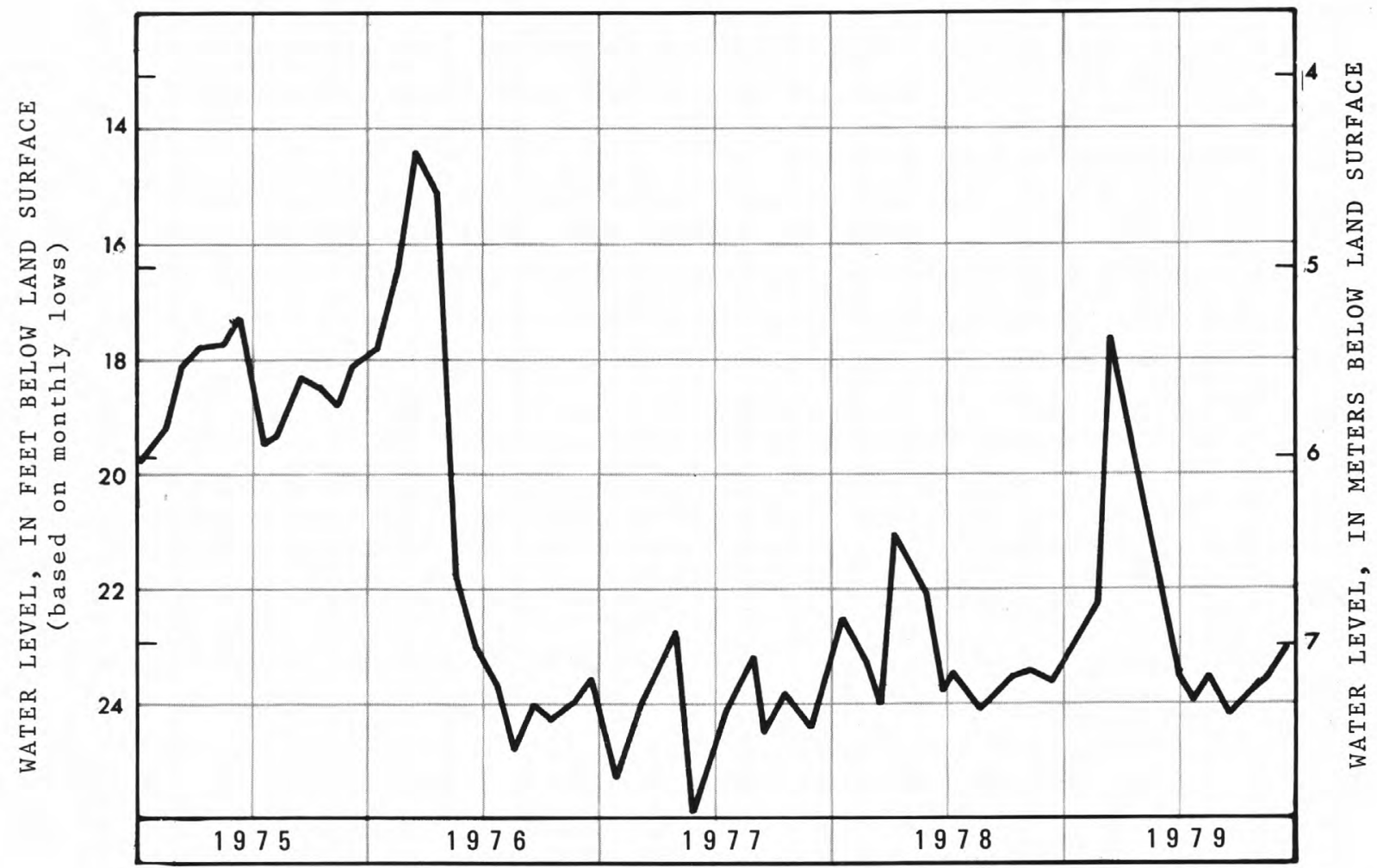

Water levels in observation well 6S 6W 22CA at Coldwater. We11 i.s 113 feet deep and in glacial deposits. 
SUPPLY AND SOURCE - - 29 we11s, 110 to 152 feet deep, tap sandstones of Marshall Formation. All are located at the Verona Field.

YIELD OF WELLS - - 300 to 1,000 gal/min; specific capacity -- 50 to 650 $\mathrm{ga} 1 / \mathrm{min} / \mathrm{ft}$ of drawdown.

PUMPAGE - - Total annual pumpåge, in million gallons, for past 5 years.

$$
\begin{aligned}
& 1979-2,875 \\
& 1978-2,659 \\
& 1977-2,443 \\
& 1976-2,357 \\
& 1975-2,224
\end{aligned}
$$
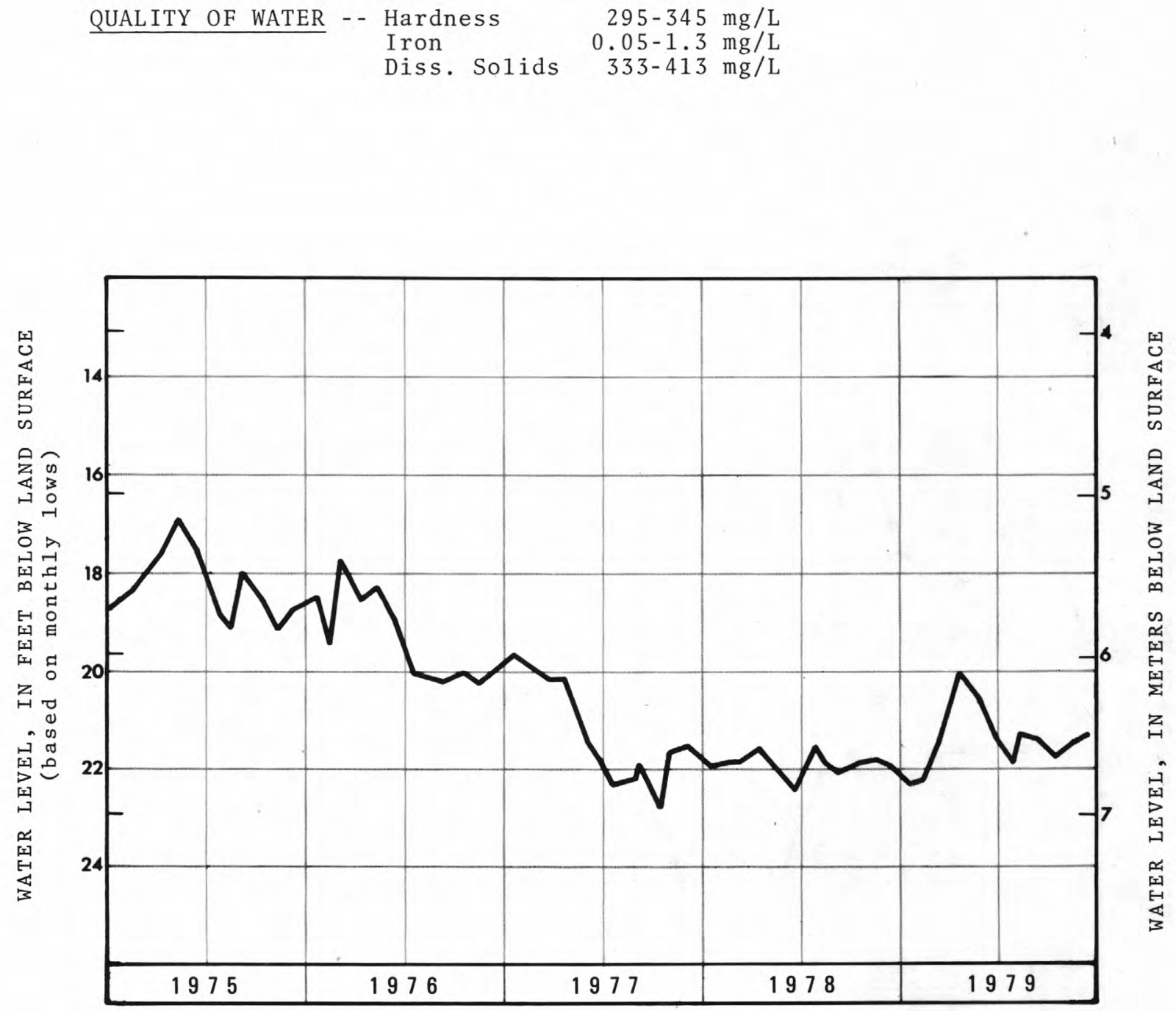

Water levels in observation we11 1S 7W 32BDCC1 at Battle Creek. Well is 95 feet deep and in the Marshall Formation. 
SUPPLY AND SOURCE -- 7 we11s, 420 to 525 feet deep, tap sandstones of Saginaw Formation.

YIELD OF WELLS -- 250 to $500 \mathrm{ga} 1 / \mathrm{min}$; specific capacity -- 3 to $12 \mathrm{ga} / \mathrm{min} / \mathrm{ft}$ of drawdown.

PUMPAGE - - Tota1 annual pumpage, in million gallons, for past 5 years.

$$
\begin{aligned}
& 1979-511 \\
& 1978-529 \\
& 1977-607 \\
& 1976-562 \\
& 1975-559
\end{aligned}
$$

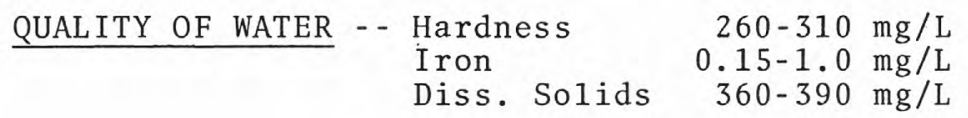

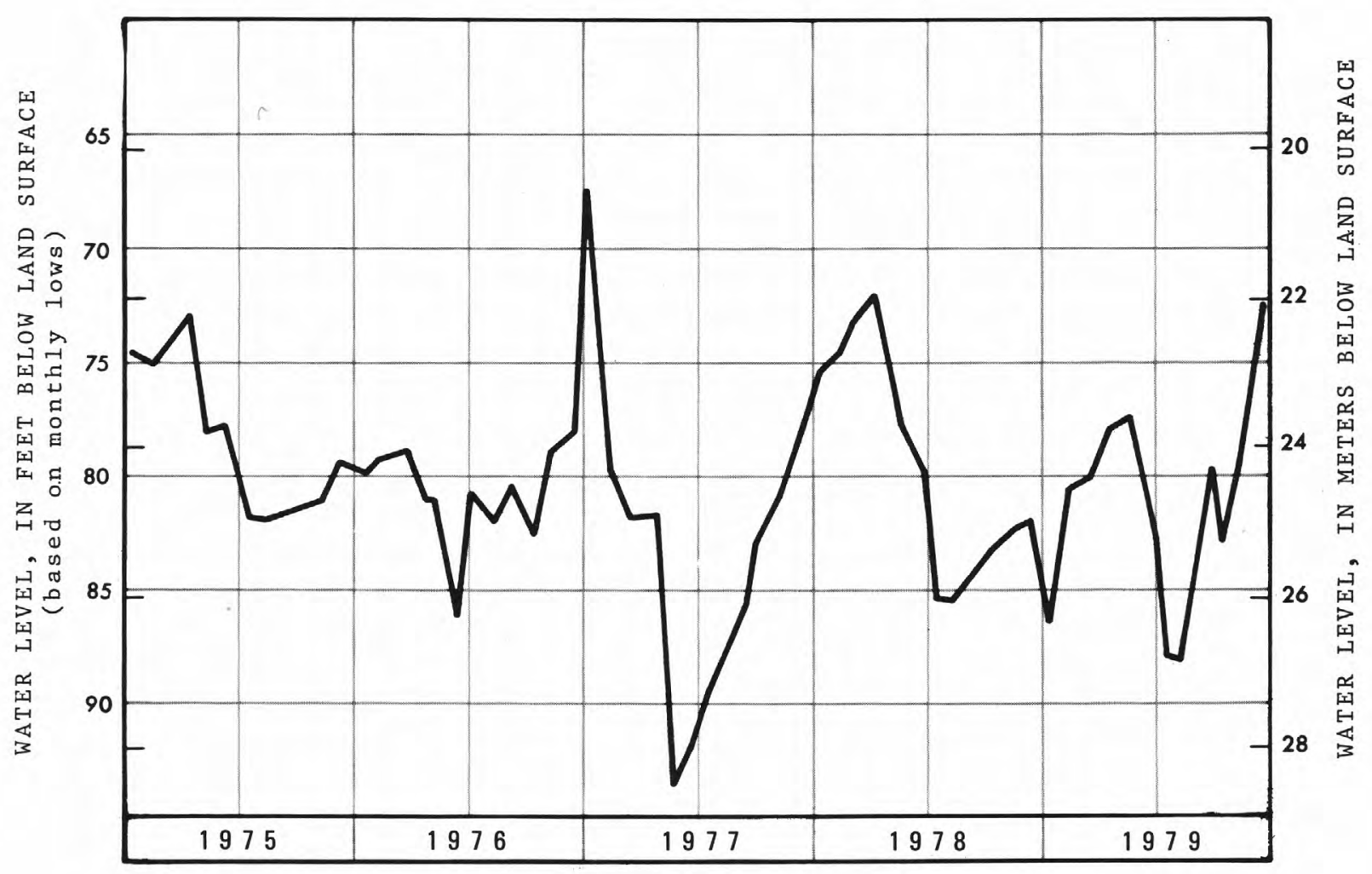

Water levels in observation well $7 \mathrm{~N} 2 \mathrm{~W} 9 \mathrm{BB}$ at St. Johns. We1l is 535 feet deep and in the Saginaw Formation. 
SUPPLY AND SOURCE - - 8 we11s, 268 to 423 feet deep, tap Saginaw Formation. YIELD OF WELLS - 160 to $700 \mathrm{ga} 1 / \mathrm{min}$.

PUMPAGE -- Total annual pumpage, in million gallons, for past 5 years. $1979-800$

$1978-771$

$1977-645$

$1976-625$

1975 - 541

QUALITY OF WATER - - Hardness

$285-429 \mathrm{mg} / \mathrm{L}$

Iron $\quad 0.5-3.1 \mathrm{mg} / \mathrm{L}$

Diss. Solids 364-539 mg/L 

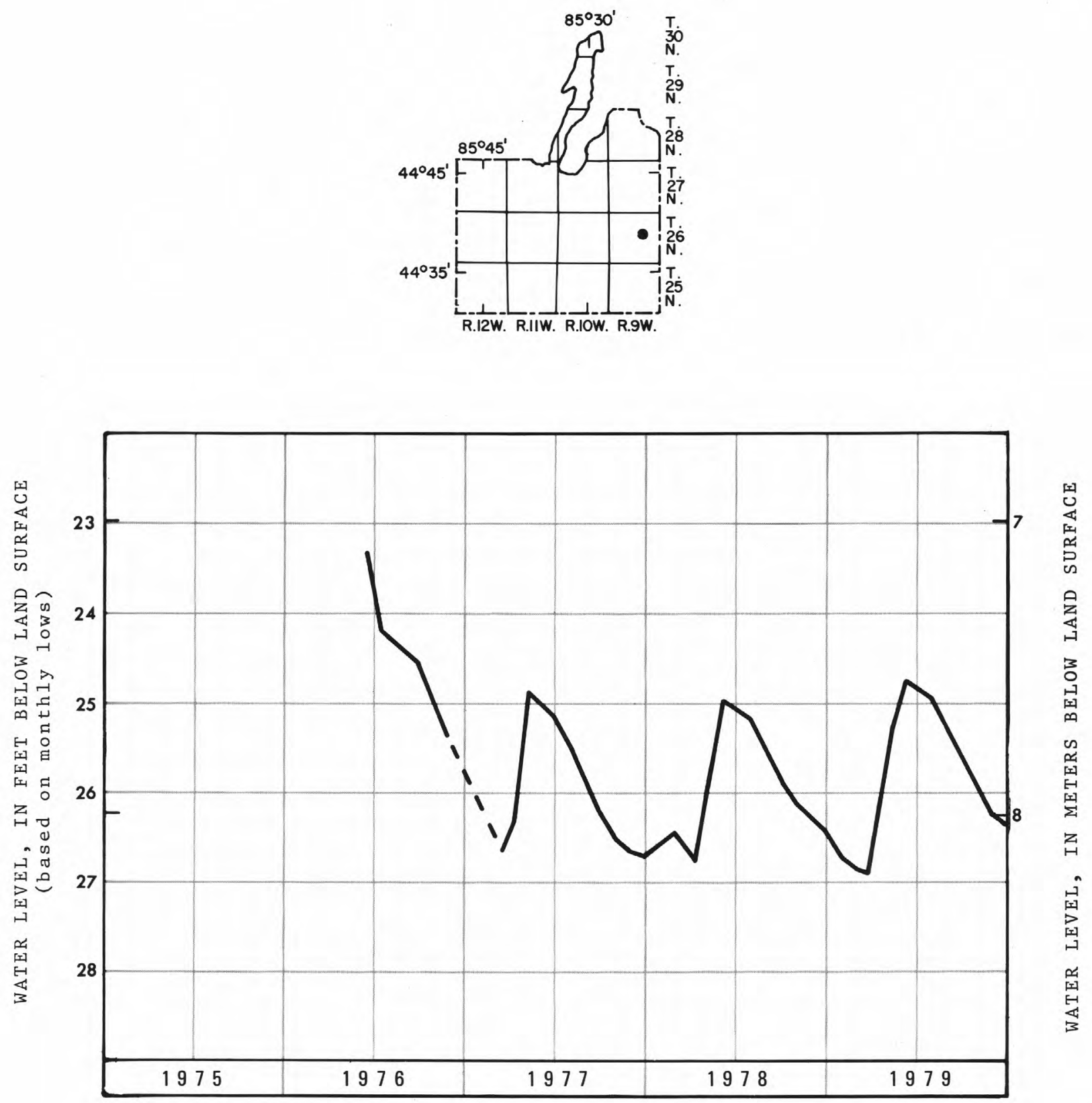

Geological Survey observation we11 26N 9W 14ABAA1. We11 is 80 feet deep and in sand. Water-quality data in previous ground-water report (Huffman, 1979). 
SUPPLY AND SOURCE - - 125 we11s, 400 to 425 feet deep, tap sandstones of Saginaw Formation; 3 we11s, 85 to 105 feet deep, tap sand beds in glacia1 deposits.

YIELD OF WELLS - - Sandstone - 100 to $700 \mathrm{ga} / \mathrm{min}$; specific capacity - 3 to $10 \mathrm{ga1} / \mathrm{min} / \mathrm{ft}$ of drawdown.

-- Glacial deposits - 790 to 1,200 ga1/min, specific capacity 12 to $80 \mathrm{ga} 1 / \mathrm{min} / \mathrm{ft}$ of drawdown.

PUMPAGE -- Tota1 annua1 pumpage, in mil1ion gallons, for past 5 years.

$$
\begin{aligned}
& 1979-9,574 \\
& 1978-9,308 \\
& 1977-9,203 \\
& 1976-8,976 \\
& 1975-8,099
\end{aligned}
$$

QUALITY OF WATER - - Composite at Plant

$$
\begin{array}{lr}
\text { Hardness } & 385 \mathrm{mg} / \mathrm{L} \\
\text { Iron } & 0.88 \mathrm{mg} / \mathrm{L} \\
\text { Diss. Solids } & 456 \mathrm{mg} / \mathrm{L}
\end{array}
$$

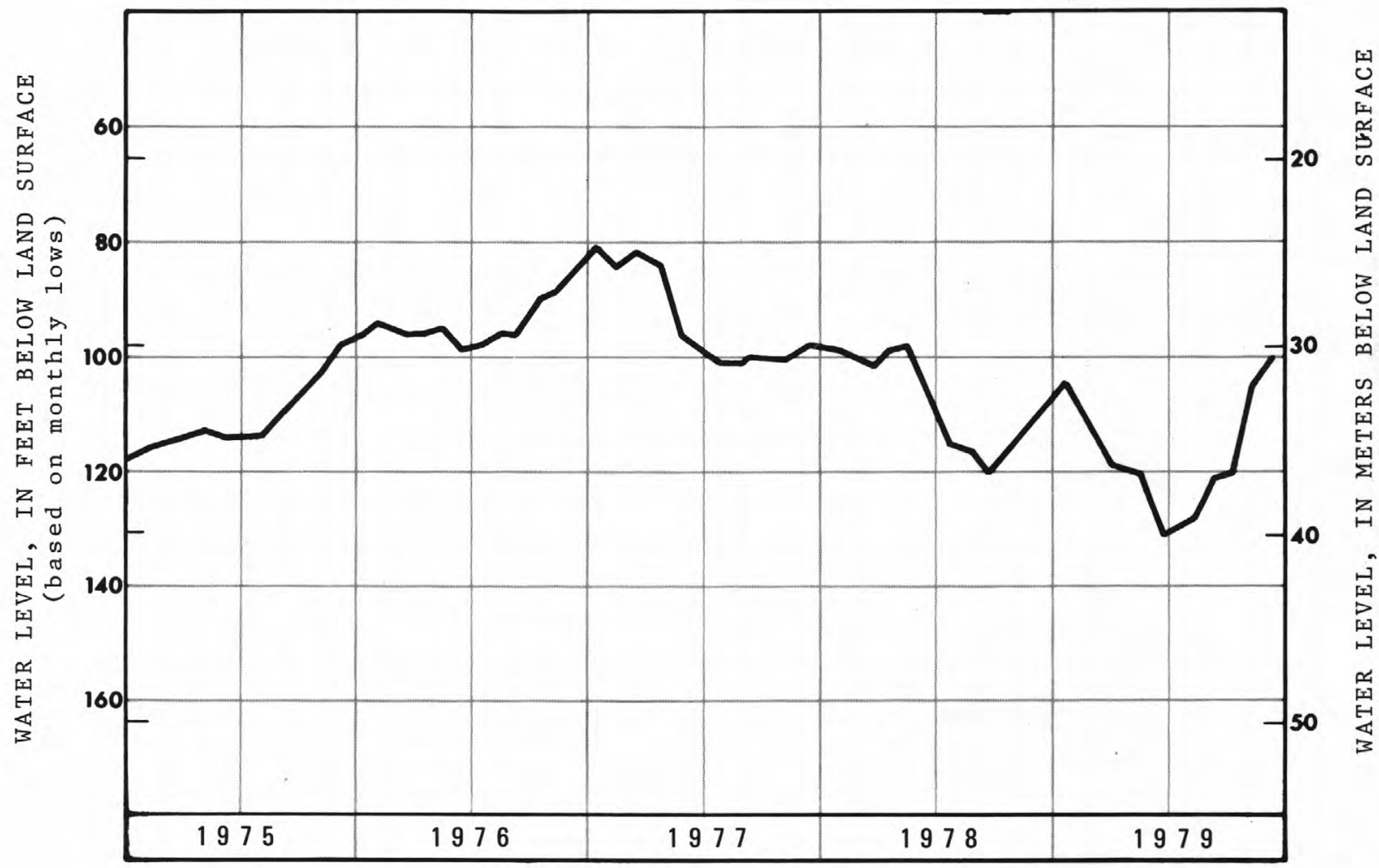

Water levels in observation we11 4N 2W 9BD at Lansing. We11 is 401 feet deep and in the Saginaw Formation. 
SUPPLY AND SOURCE - - 1 we11, about 50 feet deep, taps glacial deposits;

1 we11, 223 feet deep, taps sandstones of Saginaw Formation.

YIELD OF WELLS - 675 to 700 gal/min; specific capacity -- No. 3 yields $30 \mathrm{gal} / \mathrm{min} / \mathrm{ft}$ of drawdown from the glacial drift.

PUMPAGE -- Total annual pumpage, in million gallons, for past 5 years.

$$
\begin{aligned}
& 1979-230 \\
& 1978-191 \\
& 1977-206 \\
& 1976-218 \\
& 1975-211
\end{aligned}
$$

QUALITY OF WATER - - Hardnes S

Iron

$315-420 \mathrm{mg} / \mathrm{L}$

Diss. Solids

$0.0-0.37 \mathrm{mg} / \mathrm{L}$

$386-557 \mathrm{mg} / \mathrm{L}$

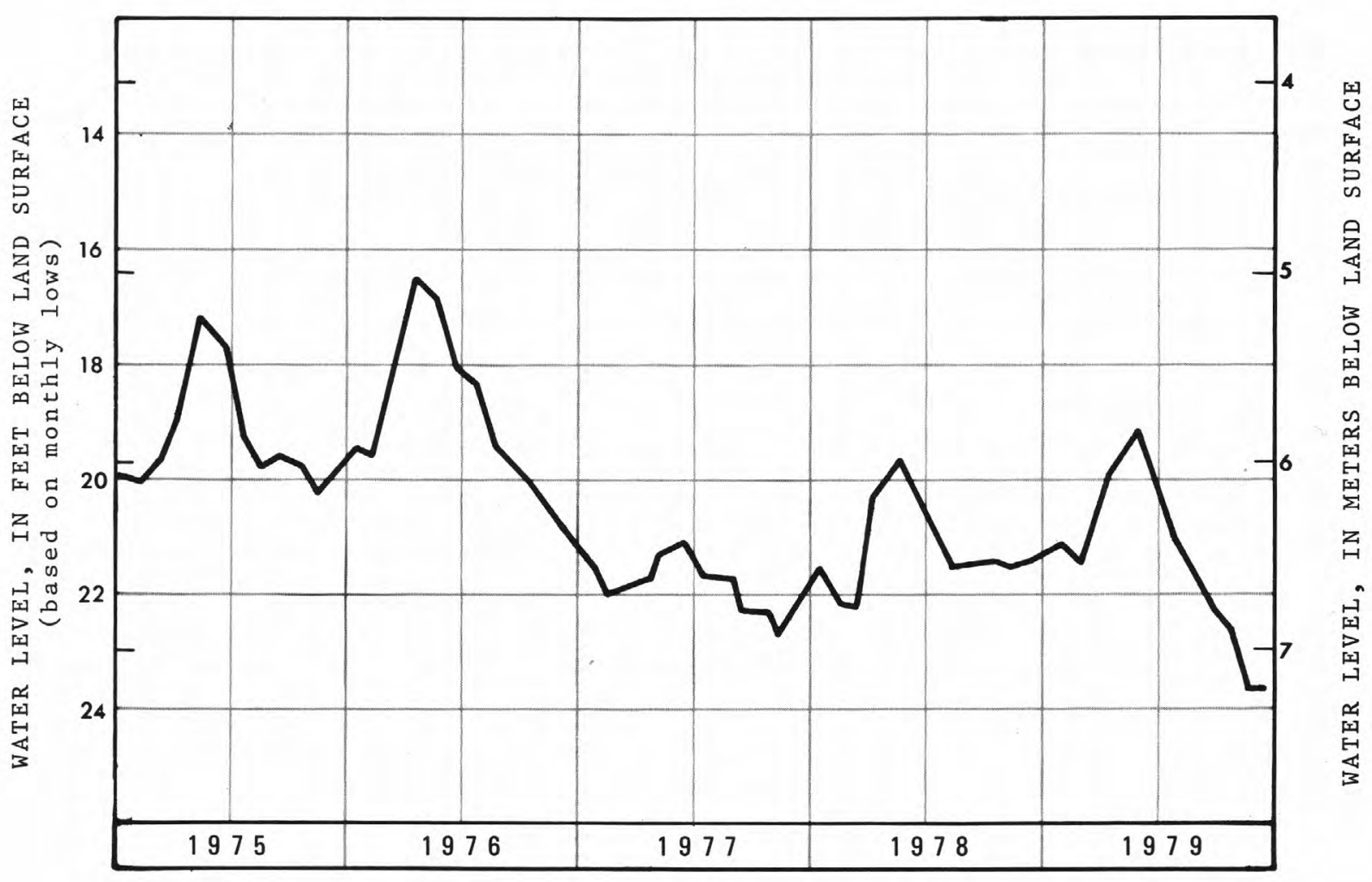

Water levels in observation we11 2N 1W 5BCAB1 at Mason. We11 is 210 feet deep and in the Saginaw Formation. 
SUPPLY AND SOURCE -- 24 we11s, 295 to 422 feet deep, tap Saginaw Formation; 1 well taps glacial deposits.

YIELD OF WELLS -- About 280 to $1,000 \mathrm{ga} 1 / \mathrm{min}$; specific capacity 2 to 12 $\mathrm{gal} / \mathrm{min} / \mathrm{ft}$ of drawdown.

PUMPAGE -- Total annual pumpage, in million gallons.

$1979-1,775$

$1978-1,702$

1977 - 1,654

$1976-1,599$

$1975-1,566$

QUALITY OF WATER - - HardnesS

$310-505 \mathrm{mg} / \mathrm{L}$

Iron

$0.9-3.8 \mathrm{mg} / \mathrm{L}$

Diss. Solids

345-662 mg/L 
SUPPLY AND SOURCE - - 7 we11s, 399 to 440 feet deep, tap sandstones of Saginaw Formation.

YIELD OF WELLS -- 260 to $500 \mathrm{ga} 1 / \mathrm{min}$; specific capacity - 3 to $8 \mathrm{ga} / \mathrm{min} / \mathrm{ft}$ of drawdown.

PUMPAGE - Total annual pumpage, in million gallons, for past 5 years. $1979-532$

$1978-538$

$1977-508$

$1976-586$

$1975-725$

QUALITY OF WATER -- Hardness

Iron

$274-435 \mathrm{mg} / \mathrm{L}$

Diss. Solids

$0.35-13.0 \mathrm{mg} / \mathrm{L}$ $320-528 \mathrm{mg} / \mathrm{L}$

REMARKS - - Most ground water pumped by the township is used to supply industrial plants in the area. 
SUPPLY AND SOURCE - - 19 we11s, 347 to 435 feet deep, tap sandstones of Saginaw் Formation; 2 wells are on a standby basis only.

YIELD OF WELLS - - 147 to $654 \mathrm{gal} / \mathrm{min}$; specific capacity - 1 to $11 \mathrm{gal} / \mathrm{min} / \mathrm{ft}$ of drawdown.

PUMPAGE -- Tota1 annual pumpage, in million gallons, for past 5 years. $1979-1,679$

$1978-1,698$

1977 - 1,675

1976 - 1,731

$1975-1,800$

QUALITY OF WATER - - Hardness

Diss. Solids

$315-350 \mathrm{mg} / \mathrm{L}$
$0.15-1.20 \mathrm{mg} / \mathrm{L}$
$361-405 \mathrm{mg} / \mathrm{L}$ 
SUPPLY AND SOURCE - - 14 we11s, 380 to 400 feet deep, tap sandstones of Saginaw and Marshal1 Formations.

YIELD OF WELLS - - 1,000 to $2,800 \mathrm{gal} / \mathrm{min}$; specific capacity - - No. 12 we 11 is $56 \mathrm{gal} / \mathrm{min} / \mathrm{ft}$ of drawdown; reported average of a11 wells is 100 $\mathrm{gal} / \mathrm{min} / \mathrm{ft}$ of drawdown.

PUMPAGE - - Total annual pumpage, in million gallons, for past 5 years.

$1979-3,993$

$1978-4,225$

$1977-4,330$

$1976-4,104$

$1975-4,077$

QUALITY OF WATER - - Hardnes S

$330-630 \mathrm{mg} / \mathrm{L}$

$0.2-0.43 \mathrm{mg} / \mathrm{L}$

Diss. Solids 394-1072 mg/L

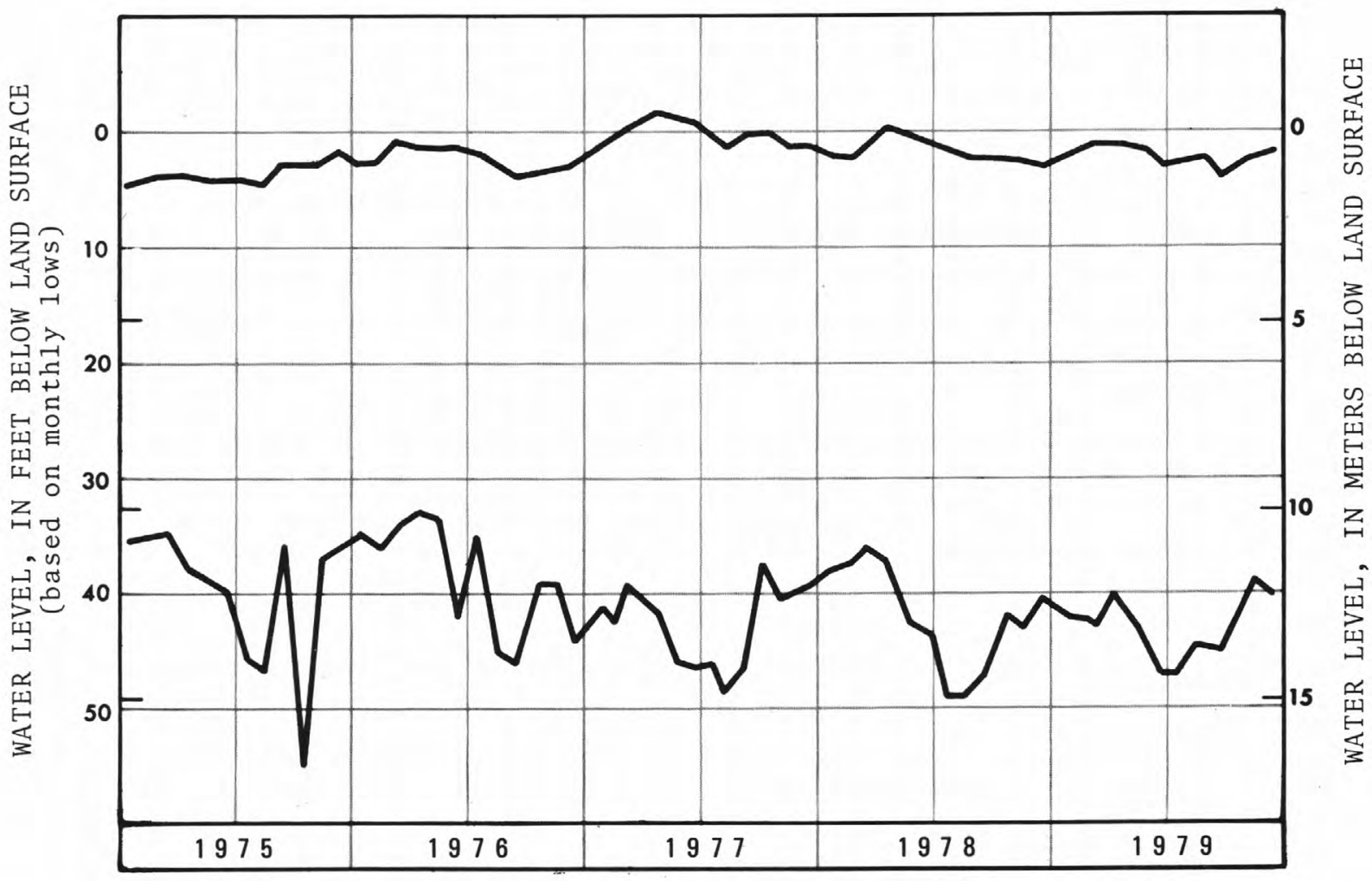

Water levels in observation wells $3 \mathrm{~S} 1 \mathrm{~W}$ 11AADD2 (top), and 3S 1W 2BDBA1 (bottom). We11 3S 1W 11AADD2 is 36 feet deep and in outwash. We11

$3 S 1 W$ 2BDBAl is 400 feet deep and in the Saginaw and Marshali Formations. 
SUPPLY AND SOURCE - - 84 we11s, 130 to 254 feet deep, tap glacial deposits. YIELD OF WELLS -- 200 to $2,000 \mathrm{ga} 1 / \mathrm{min}$; specific capacity - - 7 to 100 $\mathrm{ga} 1 / \mathrm{min} / \mathrm{ft}$ of drawdown.

PUMPAGE -- Total annual pumpage, in million gallons, for past 5 years. $1979-6,520$

$1978-6,551$

$1977-6,476$

$1976-6,549$

$1975-6,336$

QUALITY OF WATER -- Composite of 2 pumping stations:

Hardness $\quad 385-420 \mathrm{mg} / \mathrm{L}$

Iron $\quad 0.49-3.10 \mathrm{mg} / \mathrm{L}$

Diss. Solids 499-530 $\mathrm{mg} / \mathrm{L}$

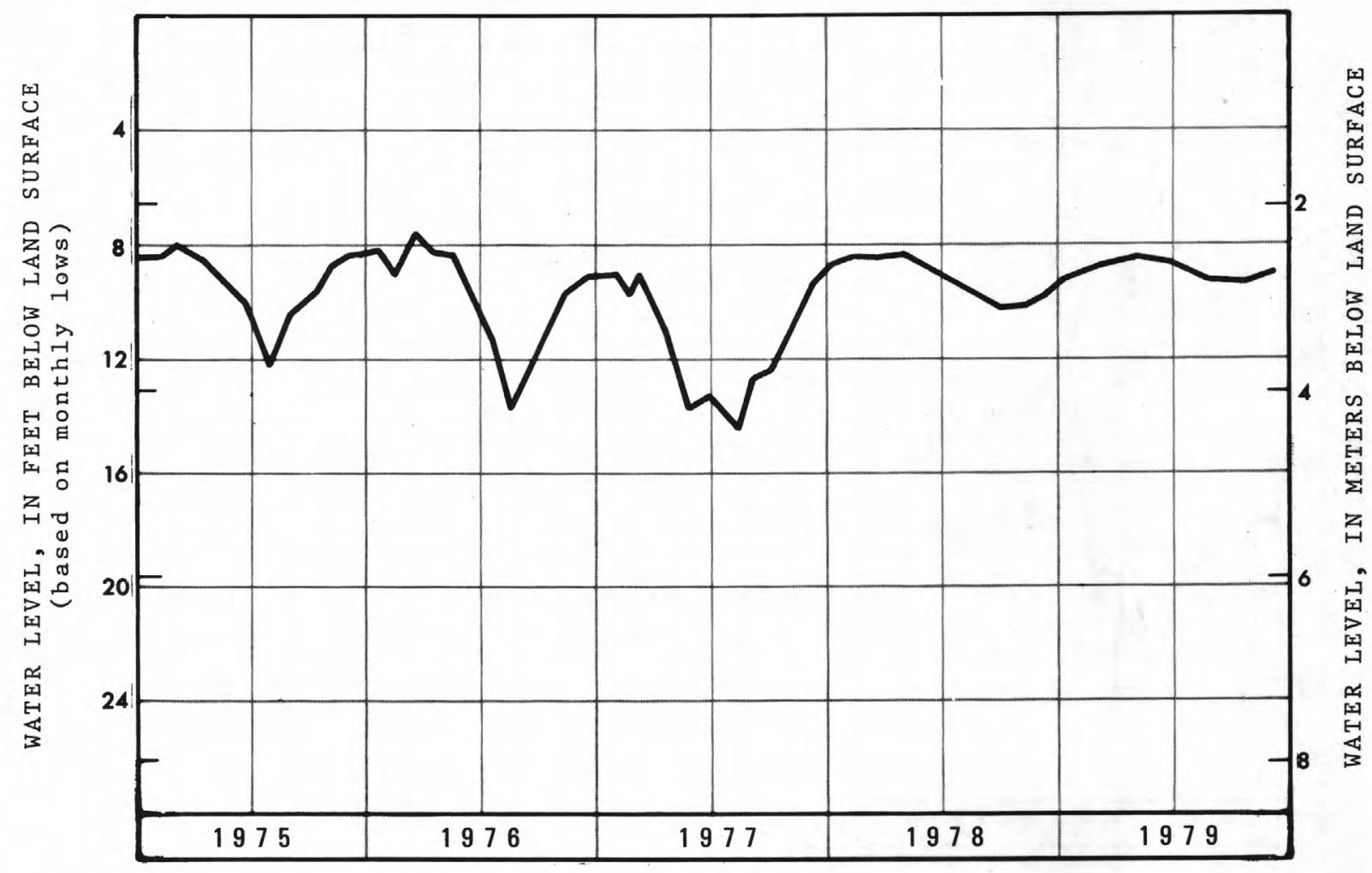

Water levels in observation well 2S $11 \mathrm{~W} 22 \mathrm{CD}$ at Kalamazoo. We11 is 137 feet deep and in outwash. 
SUPPLY AND SOURCE -- 15 we11s, 92 to 184 feet deep, tap glacia1 deposits. YIELD OF WELLS - 300 to $1,000 \mathrm{gal} / \mathrm{min}$; specific capacity -- $25 \mathrm{gal} / \mathrm{min} / \mathrm{ft}$ of drawdown.

PUMPAGE -- Total annual pumpage, in million gallons, for past 5 years.

$$
\begin{aligned}
& 1979-893 \\
& 1978-851 \\
& 1977-802 \\
& 1976-784 \\
& 1975-631
\end{aligned}
$$

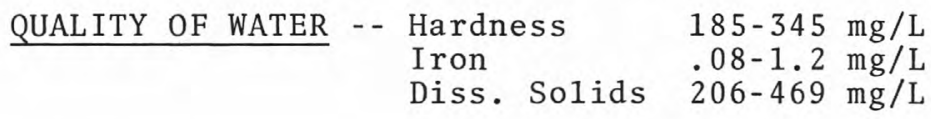

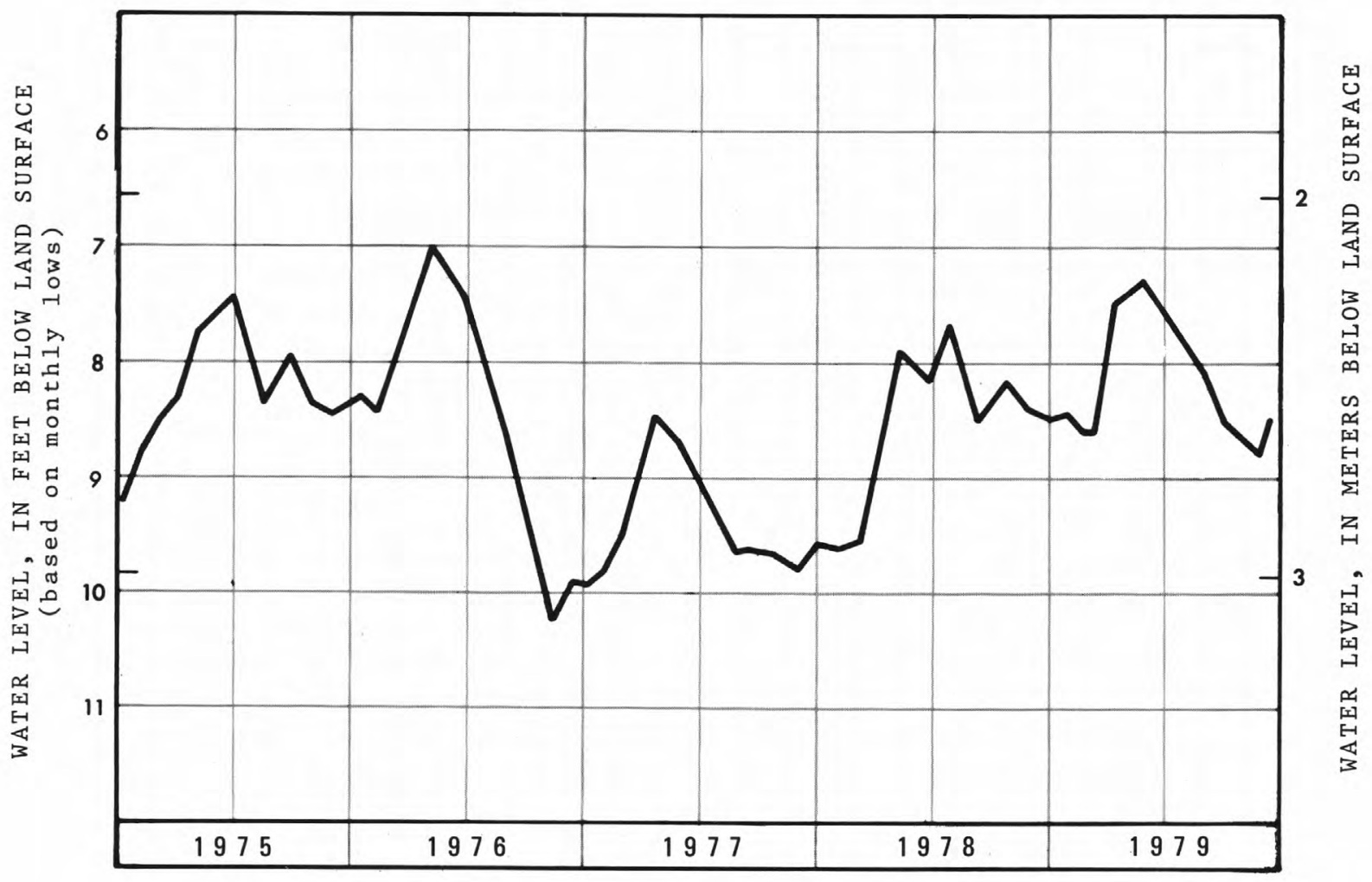

Water 1eve1s in observation we11 3S 11W 22BD at Portage. We11 is 120 feet deep and in outwash. 

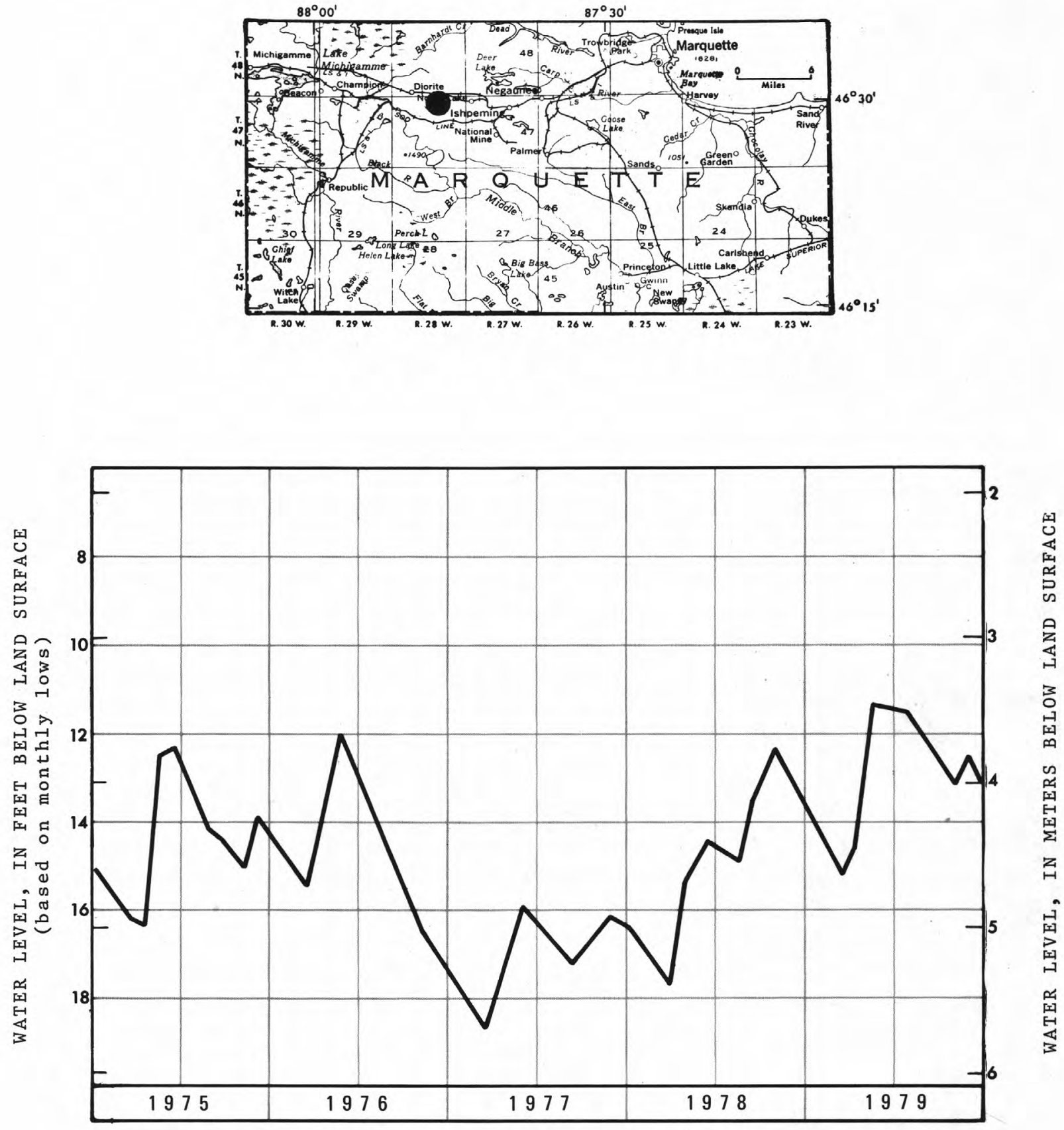

Water levels in observation well 47N 28W 3CCDCl in Marquette County. We11 is 75 feet deep and in outwash. Leve1s shown are typical of observation wells located in the Marquette Iron Range. 

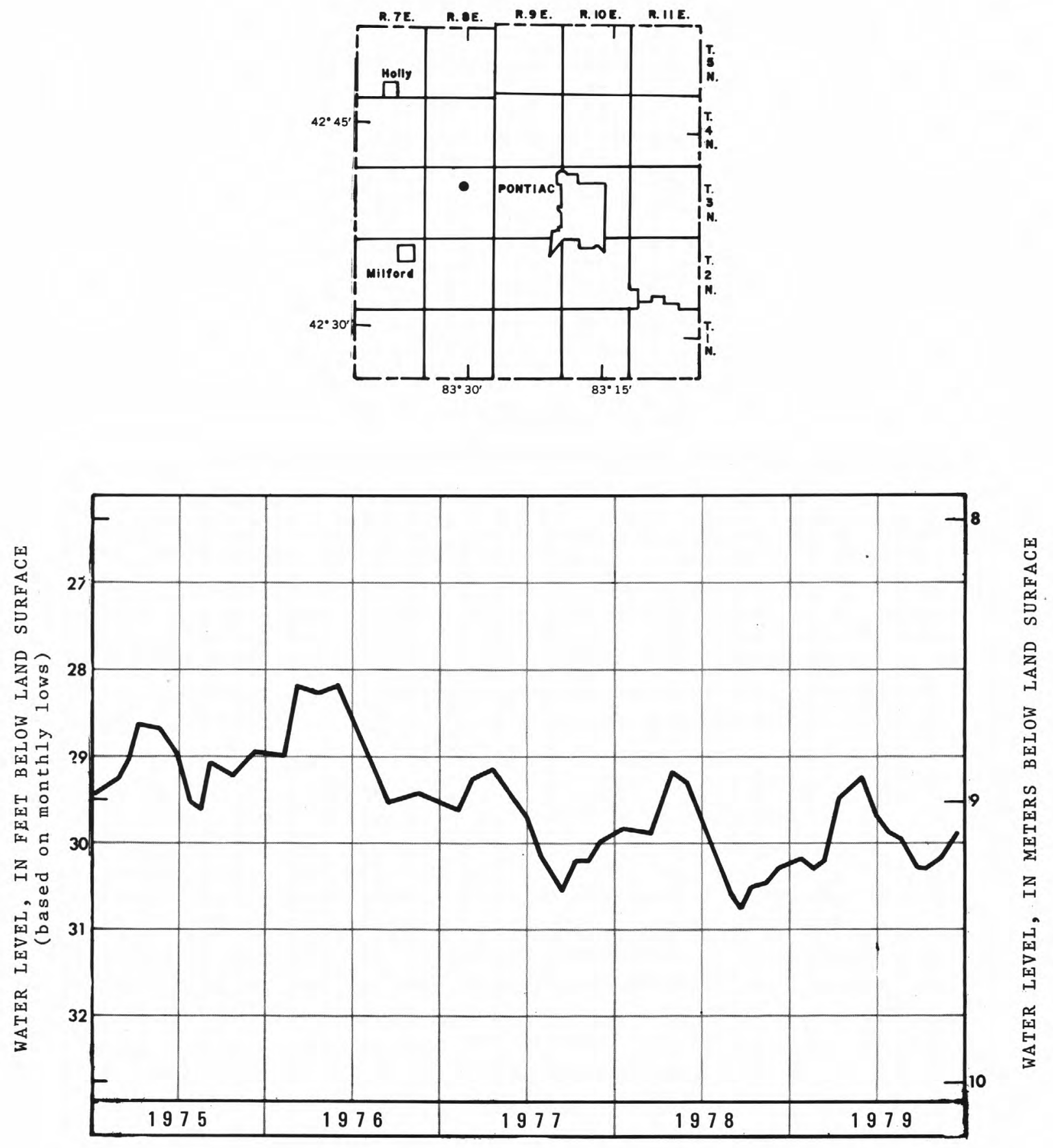

Water leve1s in observation we11 $3 \mathrm{~N} 8 \mathrm{E} 10 \mathrm{AB}$. We1l is 163 feet deep and in glacial deposits. 

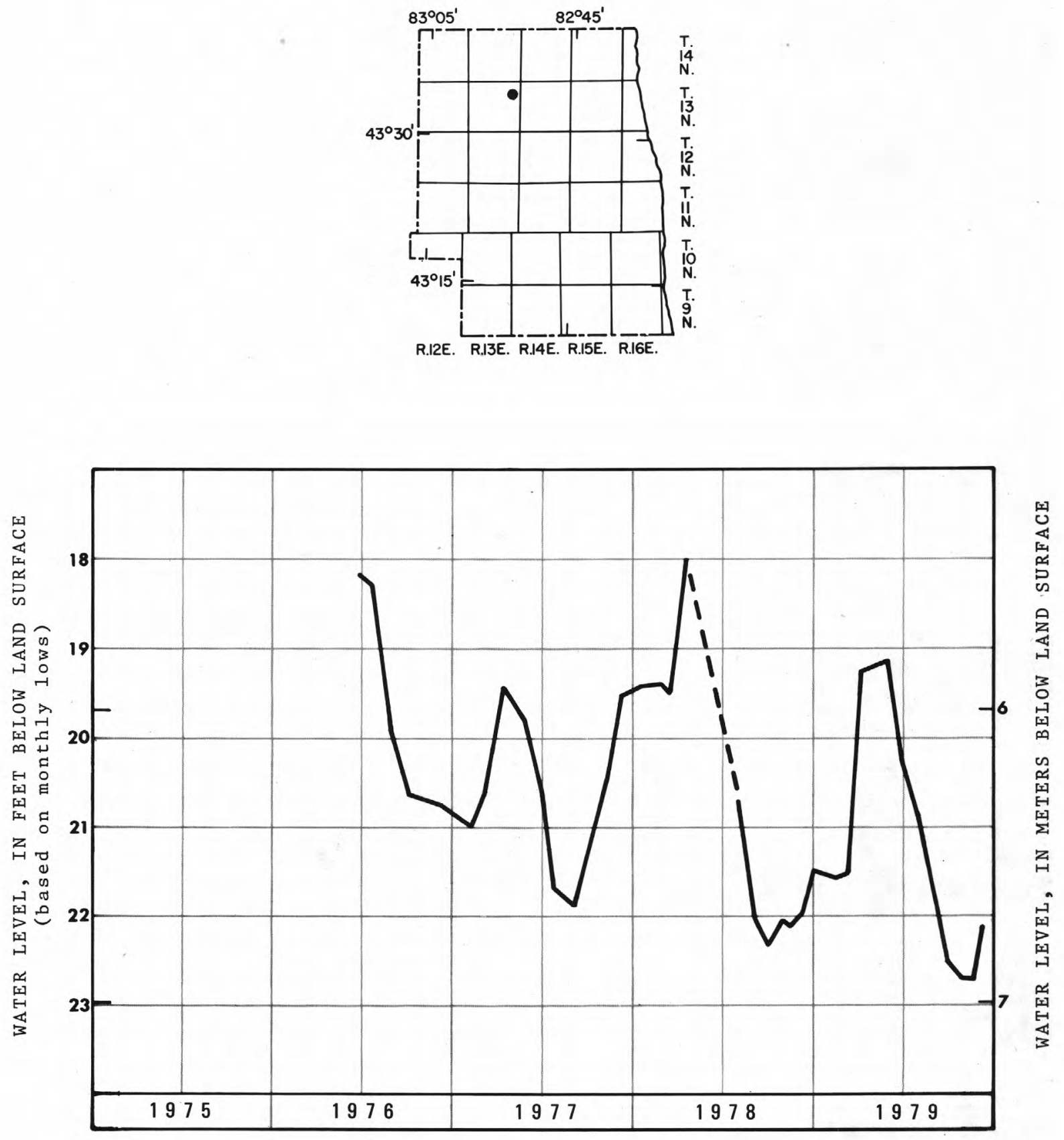

Geological Survey observation we11 13N 13E 12ADAA1. We11 is 130 feet deep and in the Marsha11 Formation. Water-quality data in previous ground-water report (Huffman, 1979). 

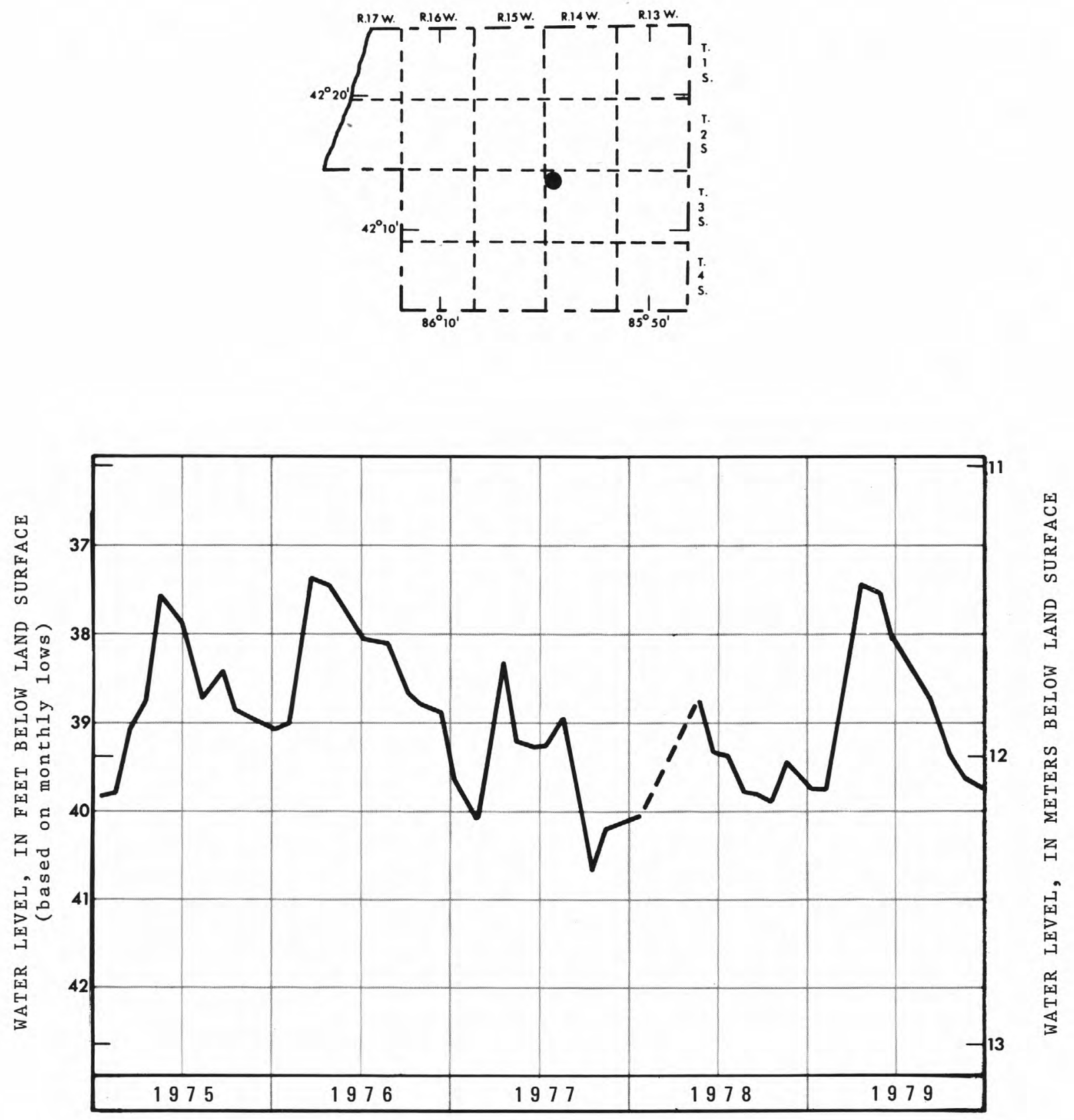

Water levels in observation we11 3S 14W 6BA in Van Buren County. We11 is 59 feet deep and in glacial deposits. Levels are typical of other observation wells located in the county. 
SUPPLY AND SOURCE -- 3 wells, 91 to 196 feet deep, tap glacial deposits; most water is pumped from the Huron River.

YIELD OF WELLS -- 1,050 to $4,860 \mathrm{ga} / \mathrm{min}$; specific capacity -- 20 to 600 $\mathrm{gal} / \mathrm{min} / \mathrm{ft}$ of drawdown.

PUMPAGE -- Total annual ground-water pumpage, in million gallons, for past 5 years (ground water is used to augment supply from Huron River).

$$
\begin{aligned}
& 1979-372 \\
& 1978-705 \\
& 1977-674 \\
& 1976-677 \\
& 1975-878
\end{aligned}
$$

QUALITY OF WATER -- Ground water;

$\begin{array}{lr}\text { Hardness } & 355-585 \mathrm{mg} / \mathrm{L} \\ \text { Iron } & 0.25-2.4 \mathrm{mg} / \mathrm{L}\end{array}$

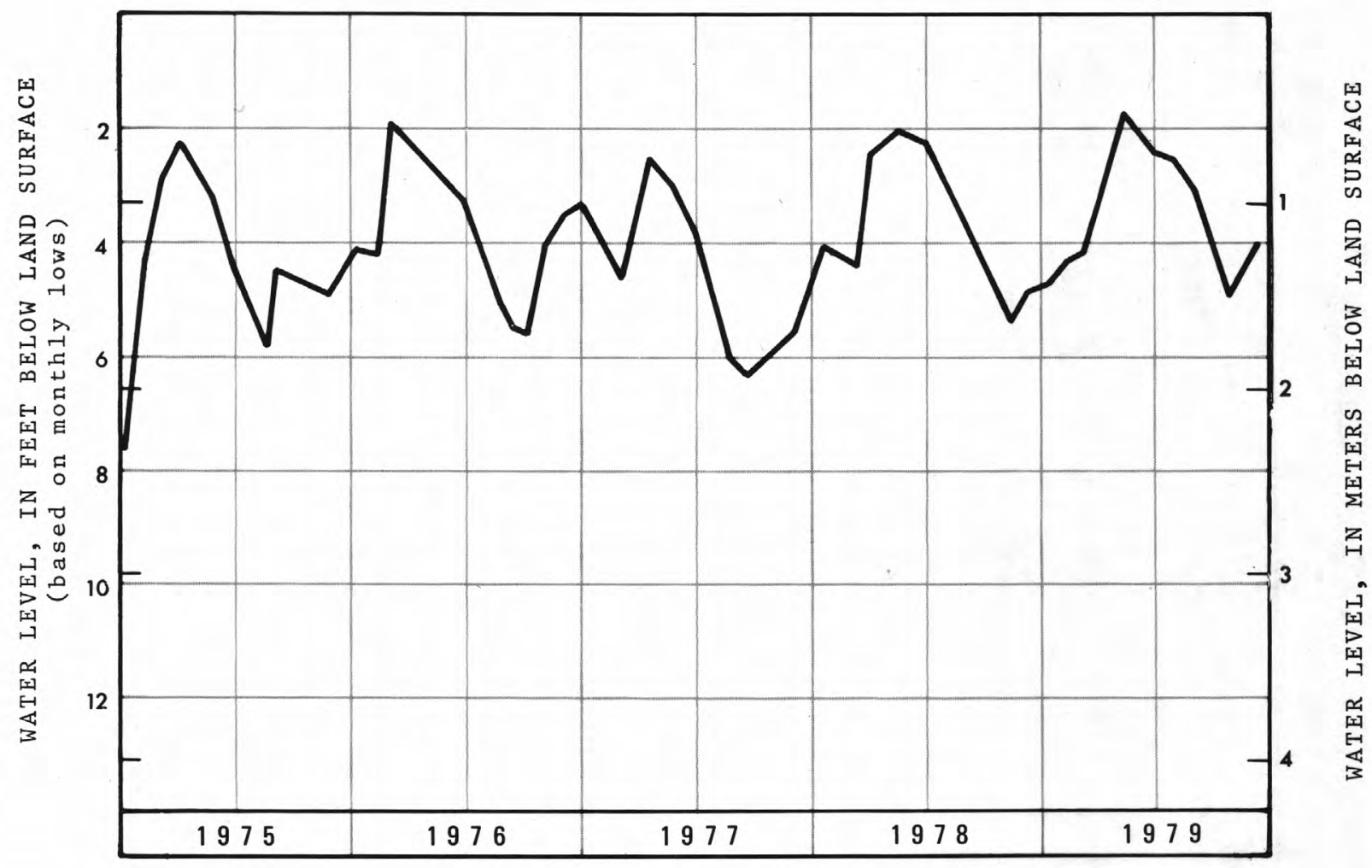

Water leve1s in observation we11 3S 6E 16BCCD1 at Ann Arbor. We11 is 55 feet deep and in glacial deposits. 
SUPPLY AND SOURCE -- 6 we11s, 87 to 102 feet deep, tap glacial deposits. YIELD OF WELLS -- Average $450 \mathrm{ga} 1 / \mathrm{min}$; specific capacity -- 25 to 180 gal/min/ft of drawdown.

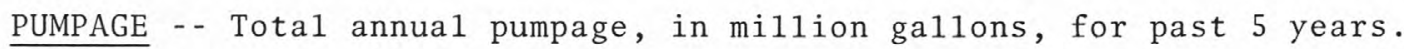

$$
\begin{aligned}
& 1979-1,140 \\
& 1978-1,296 \\
& 1977-1,440 \\
& 1976-1,652 \\
& 1975-1,462
\end{aligned}
$$

QUALITY OF WATER -- Composite

$\begin{array}{ll}\text { Hardness } & 355 \mathrm{mg} / \mathrm{L} \\ \text { Iron } & 0.2 \mathrm{mg} / \mathrm{L} \\ \text { Diss. Solids } & 475 \mathrm{mg} / \mathrm{L}\end{array}$

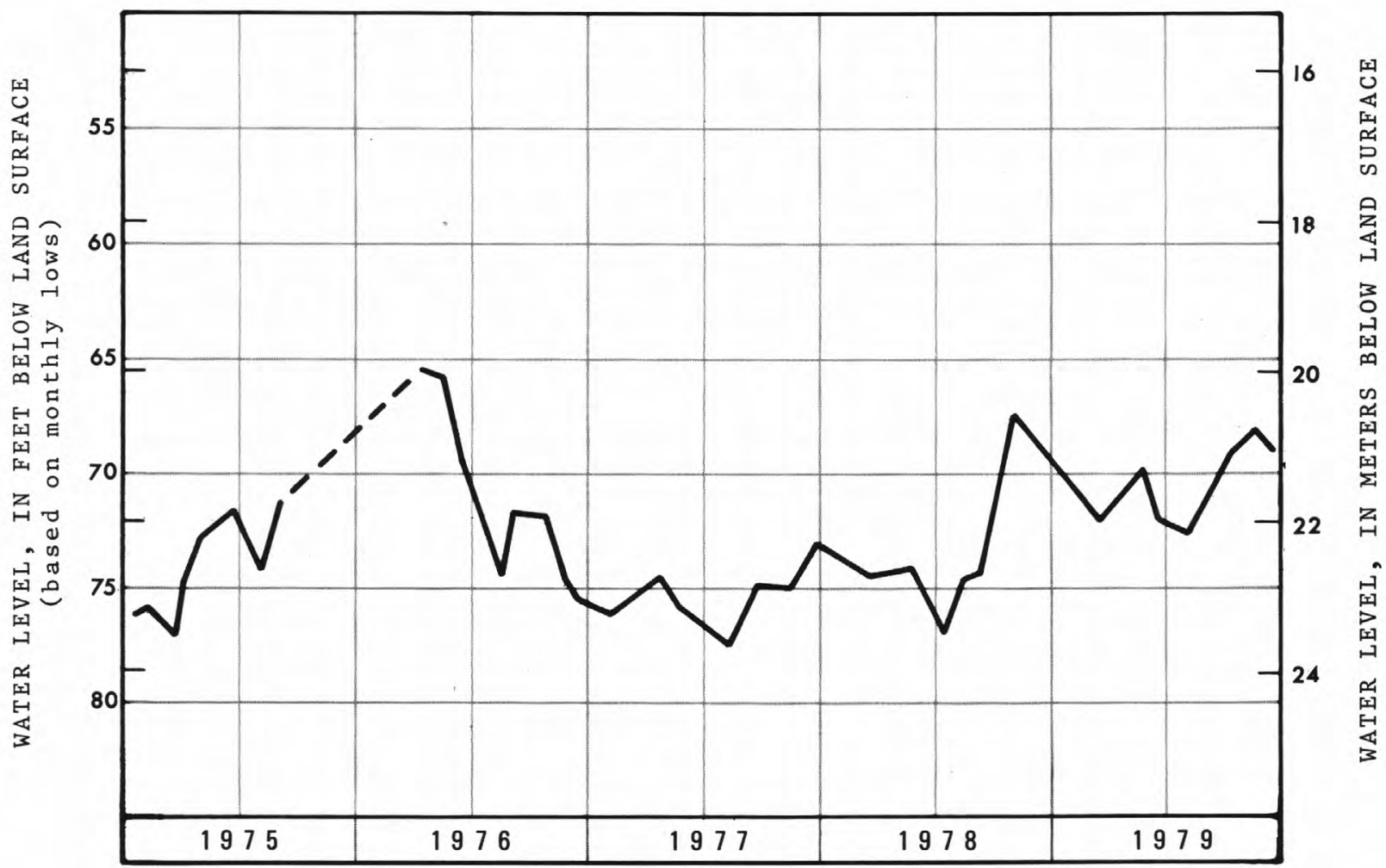

Water levels in observation we11 3S $7 \mathrm{E} 9 \mathrm{AD}$ at Ypsilanti. We11 is 94 feet deep and in glacial deposits. 
SUPPLY AND SOURCE -- 9 we11s, 50 to 95 feet deep, tap glacial deposits. YIELD OF WELLS -- 700 to $3,500 \mathrm{gal} / \mathrm{min}$.

PUMPAGE - Total annual pumpage, in million gallons, for past 5 years.

$\begin{array}{rr}1979- & 431 \\ 1978- & 986 \\ 1977- & 792 \\ 1976-1,067 \\ 1975- & 854\end{array}$

QUALITY OF WATER - - Composite

$\begin{array}{ll}\text { Hardness } & 350 \mathrm{mg} / \mathrm{L} \\ \text { Iron } & 1.4 \mathrm{mg} / \mathrm{L} \\ \text { Diss. Solids } & 477 \mathrm{mg} / \mathrm{L}\end{array}$

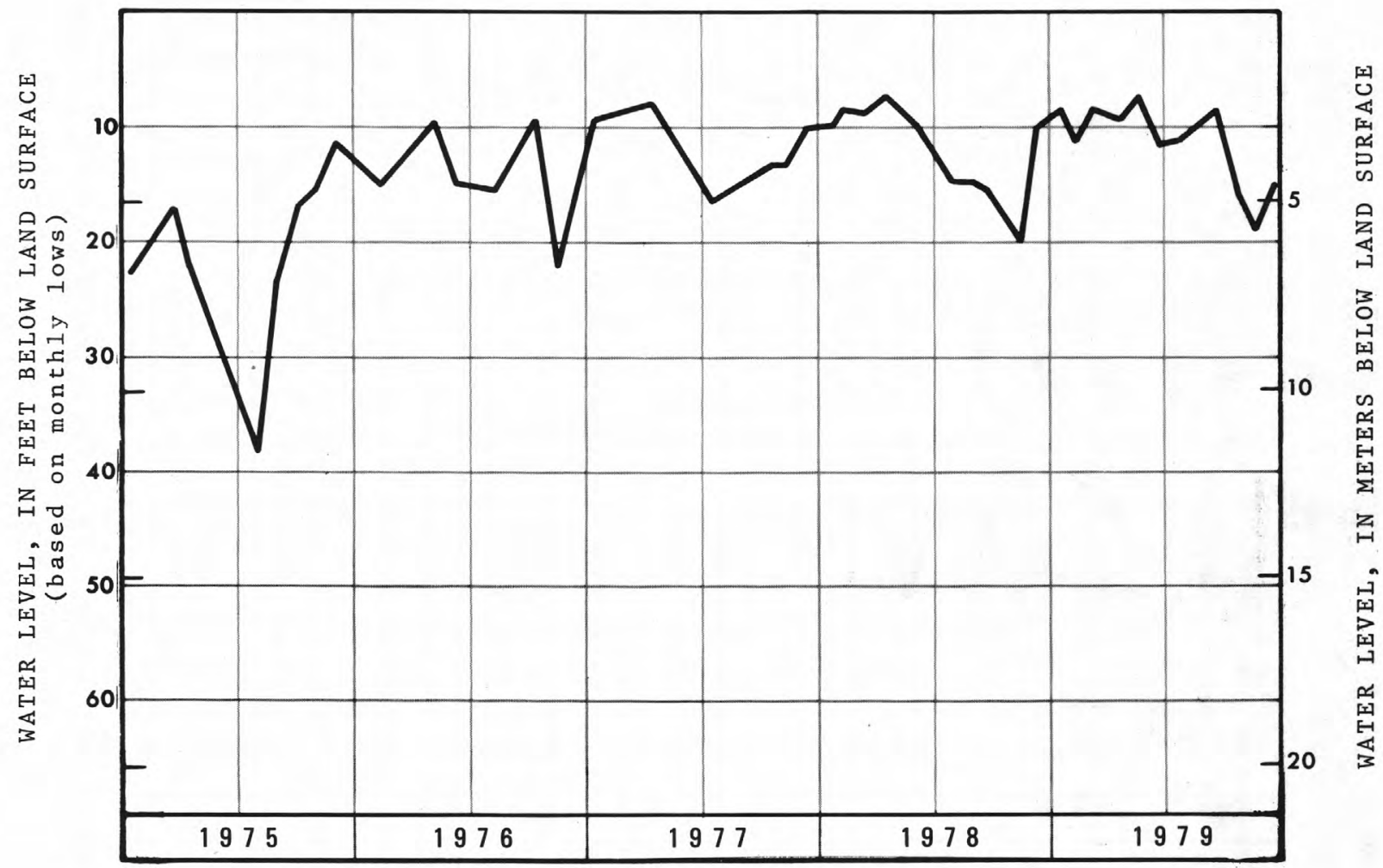

Water levels in observation well $3 \mathrm{~S} 7 \mathrm{E} 24 \mathrm{CD}$ in Ypsilanti Township. Well is 75 feet deep and in glacial deposits. 
TABLES 
COUNTY AND WELL NUMBER: See section in text entitled "Well-numbering system."

NAME: MDNR - Michigan Department of Natural Resources; WMP - Wisconsin-Michigan Power Company; MSHD - Michigan State Highway Department; USFS - U.S. Forest Service; HCMA - Huron-Clinton Metropolitan Authority; BCRC - Branch County Road Commission.

AQUIFER: 112GLCL Glacial deposits 337MRSL Marshall Formation 3610CVCU Ordovician, Upper

112GRV Gravel $\quad$ 341TRVR Traverse Group $365 T$ TRV Trenton-Black River Group

1120TSH Outwash 344DUND Dundee Formation 368PRDC Prairie du Chien Group

112SAND Sand 348DRV Detroit River Group 372MNSG Munising Sandstone

1124SG Sand and gravel 35SLINH Salina Formation 420FRED Freda Sandstone

ALTITUDE: Land-

ALTITUDE: Land-surface datum in feet above mean sea level.

MEASUREMENTS, 1979 (frequency): R - Continuous recorder; D - Daily; W - Weekly; M - Monthly; Q - Quarterly; S - Semiannually; A - Annually; I - Intermittent.

OBSERVED WATER-LEVEL EXTREMES: In feet below or above (+) land surface. 1979 measurements underscored are extremes for entire record,

REMARKS: P - Water levels affected by pumping. Water-level measurements are made by the U.S. Geological Survey unless otherwise noted.

\begin{tabular}{|c|c|c|c|c|c|c|c|c|c|c|c|c|}
\hline \multirow{3}{*}{$\begin{array}{c}\text { COUNTY } \\
\text { AND } \\
\text { WELL NUMBER } \\
\text { TWP, RANGE, SECT } \\
\end{array}$} & \multirow{3}{*}{ NAME } & \multirow{3}{*}{$\begin{array}{l}\text { ミ } \\
\text { 志 } \\
\text { 㟧 }\end{array}$} & \multirow{3}{*}{ 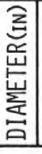 } & \multirow{3}{*}{$\begin{array}{l}\text { 岀 } \\
\text { 莺 }\end{array}$} & \multirow{3}{*}{ 菅 } & \multirow{3}{*}{$\begin{array}{l}\text { 롱 } \\
\text { 㟧 } \\
\dot{\tilde{\alpha}} \\
\dot{\alpha} \\
\end{array}$} & \multirow{3}{*}{ 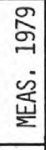 } & \multicolumn{4}{|c|}{ OBSERVED WATER-LEVEL EXTREMES } & \multirow{3}{*}{ REMARKS } \\
\hline & & & & & & & & \multicolumn{2}{|c|}{ THROUGH 1978} & \multicolumn{2}{|c|}{ IN 1979} & \\
\hline & & & & & & & & MAXIMUM & MINIMUM & MAXIMUM & MINIMUM & \\
\hline \multicolumn{13}{|l|}{$\underline{\text { ALGER }}$} \\
\hline $45 \mathrm{~N} 19 \mathrm{~W} 25 \mathrm{BD}$ & $\mathrm{CCC}$ & 66 & 6 & $112 \mathrm{GLCL}$ & 850 & 21 & Q & 6.4 Jun 1960 & 14.2 Apr 1964 & 8.7 Jun & $10.4 \mathrm{Mar}$ & \\
\hline \multicolumn{13}{|l|}{ ALPENA } \\
\hline $32 \mathrm{~N} 6 \mathrm{E} 23 \mathrm{DDDA} 1$ & Alpena State Forest & 88 & 6 & 112SAND & 713 & 3 & $\mathrm{R}$ & 17.1 May 1978 & 28.7 Mar 1977 & $\underline{15.8 \mathrm{May}}$ & 25.7 Mar & \\
\hline \multicolumn{13}{|l|}{ BARAGA } \\
\hline $48 \mathrm{~N} 32 \mathrm{~W} 12 \mathrm{DD}$ & WMP 14 & 10 & 1 & 112GLCL & 1,630 & 32 & M & 3.3 Apr 1965 & 8.1 Sep 1969 & $6.0 \mathrm{Jun}$ & $6.8 \mathrm{Feb}$ & Meas. by WMP \\
\hline \multicolumn{13}{|l|}{$\underline{\text { BARRY }}$} \\
\hline 4N 9W 5DA & Solomon Road & 131 & 2 & 112GLCL & 860 & 16 & $Q$ & 111.5 Mar 1978 & $122.0 \mathrm{Mar} 1965$ & 115.1 Sep & $117.1 \mathrm{Mar}$ & \\
\hline \multicolumn{13}{|l|}{$\underline{\mathrm{BAY}}$} \\
\hline 17N 4E 22DCAA1 & Pinconning Township & 110 & 6 & $324 \mathrm{SGNW}$ & 620 & 18 & $\mathrm{R}$ & 0.0 Mar 1976 & 10.5 Aug 1963 & $1.4 \mathrm{Apr}$ & $4.8 \mathrm{Jul}$ & \\
\hline \multicolumn{13}{|l|}{ BRANCH } \\
\hline $5 S 6 W 22 B B$ & Girard & 27 & 1 & 112GLCL & 950 & 16 & M & 10.5 Mar 1974 & 16.3 Nov 1964 & $13.7 \mathrm{Jan}$ & $13.9 \mathrm{Feb}$ & Disc. 2-79 \\
\hline $8 \mathrm{~W} 28 \mathrm{DB}$ & Sherwood & 42 & 1 & 112GLCL & 880 & 15 & M & 11.3 Mar 1976 & 18.9 Nov 1965 & $17.6 \mathrm{Jan}$ & 17.7 Feb & Disc. 2-79 \\
\hline $6 \mathrm{~S} 6 \mathrm{~W} \quad 18 \mathrm{CCCD} 1$ & Coldwater Township & 56 & 6 & $1120 \mathrm{TSH}$ & 950 & 16 & M & 18.3 Mar 1976 & 28.3 Jul 1964 & $21.3 \mathrm{Sep}$ & $25.0 \mathrm{Feb}$ & \\
\hline $22 \mathrm{CA}$ & Coldwater Test 4 & 113 & 6 & $112 \mathrm{GLCL}$ & 970 & 16 & $\mathrm{R}$ & 9.0 May 1975 & 25.9 May 1977 & $10.9 \mathrm{Apr}$ & 24.2 Sep & P \\
\hline $\begin{array}{l}8 S 5 W 6 A B \\
\text { CALHOUN } \\
\end{array}$ & Chipman & 55 & 4 & 112GLCL & 1,032 & 16 & M & 13.9 Feb 1968 & 19.4 Dec 1964 & $17.4 \mathrm{Jan}$ & $17.6 \mathrm{Feb}$ & Disc. 2-79 \\
\hline \multirow{2}{*}{$\begin{array}{l}\text { 1S } 7 \mathrm{~W} 10 \mathrm{BB} \\
32 \mathrm{BDCC} 1\end{array}$} & Sabin & 12 & 15 & 112GLCL & 908.0 & 34 & W & 0.9 Mar 1950 & 7.2 Dec 1964 & $2.7 \mathrm{Apr}$ & 4.7 Dee & Meas. by owner \\
\hline & Penfield Township & 95 & 6 & 337MRSL & 845 & 16 & $\mathrm{R}$ & 15.6 Apr 1974 & 27.0 Aug 1964 & 18.2 May & $22.3 \mathrm{Jan}$ & $\mathrm{P}$ \\
\hline $32 \mathrm{DA}$ & Battle Creek & 127 & 8 & 337MRSL & 830.8 & 41 & $\mathrm{D}$ & $0.7 \mathrm{Apr} 1950$ & 16.8 Jul 1959 & 4.6 Apr & $9.8 \mathrm{Sep}$ & P, Meas. by owner \\
\hline $\begin{array}{l}2 S 6 Q 25 A A \\
\text { CASS }\end{array}$ & Marsha11 & 59 & 6 & 337MRSL & 904.8 & 30 & M & 5.5 May 1950 & 9.7 Aug 1964 & $7.8 \mathrm{Jan}$ & $8.7 \mathrm{Nov}$ & P, Meas. by owner \\
\hline $\begin{array}{l}\text { 8S 14W 17BA } \\
\text { CHEBOYGAN }\end{array}$ & Little & 55 & 28 & 112GLCL & 840 & 35 & M & 46.2 Jul 1950 & 55.0 Mar 1957 & 50.3 Aug & 52.1 Jun & \\
\hline $33 N \quad 1 W \quad 26 D A B B 1$ & Pigeon River CCC & 164 & 6 & 112 SAND & 933 & 14 & $\mathrm{R}$ & 56.2 May 1971 & $59.9 \mathrm{Dec} 1965$ & $\underline{56.1 \mathrm{Jun}}$ & $58.9 \mathrm{Feb}$ & \\
\hline $39 \mathrm{~N} 3 \mathrm{~W} \quad 29 \mathrm{CBCB} 1$ & Mackinaw 1 & 125 & 6 & 344DUND & 705 & 1 & $\mathrm{M}$ & & & 5.2 May & 9.5 Sep & \\
\hline $\begin{array}{l}\text { 39N 3W 29CBCB2 } \\
\text { CHIPPEWA }\end{array}$ & Mackinaw 2 & 55 & \multicolumn{8}{|c|}{$\underline{\text { CHIPPEWA }}$} & & \\
\hline $\begin{array}{l}\text { 46N 4W 24DADA1 } \\
\text { CLARE }\end{array}$ & Raco & 54 & 6 & $1120 \mathrm{TSH}$ & 850 & 27 & $\mathrm{R}$ & 18.4 Jun 1971 & 28.4 Apr 1964 & 20.4 May & $24.4 \mathrm{Mar}$ & \\
\hline $17 \mathrm{~N} 4 \mathrm{~W}$ 34DCAD & Clare & 91 & 4 & 112GLCL & 850 & 5 & $\mathrm{R}$ & 7.9 Mar 1976 & 24.9 May 1977 & 11.6 May & $23.1 \mathrm{Jul}$ & P \\
\hline
\end{tabular}


TABLE 1. RECORDS OF MICHIGAN OBSERVATION WELLS. (CONTINUED)

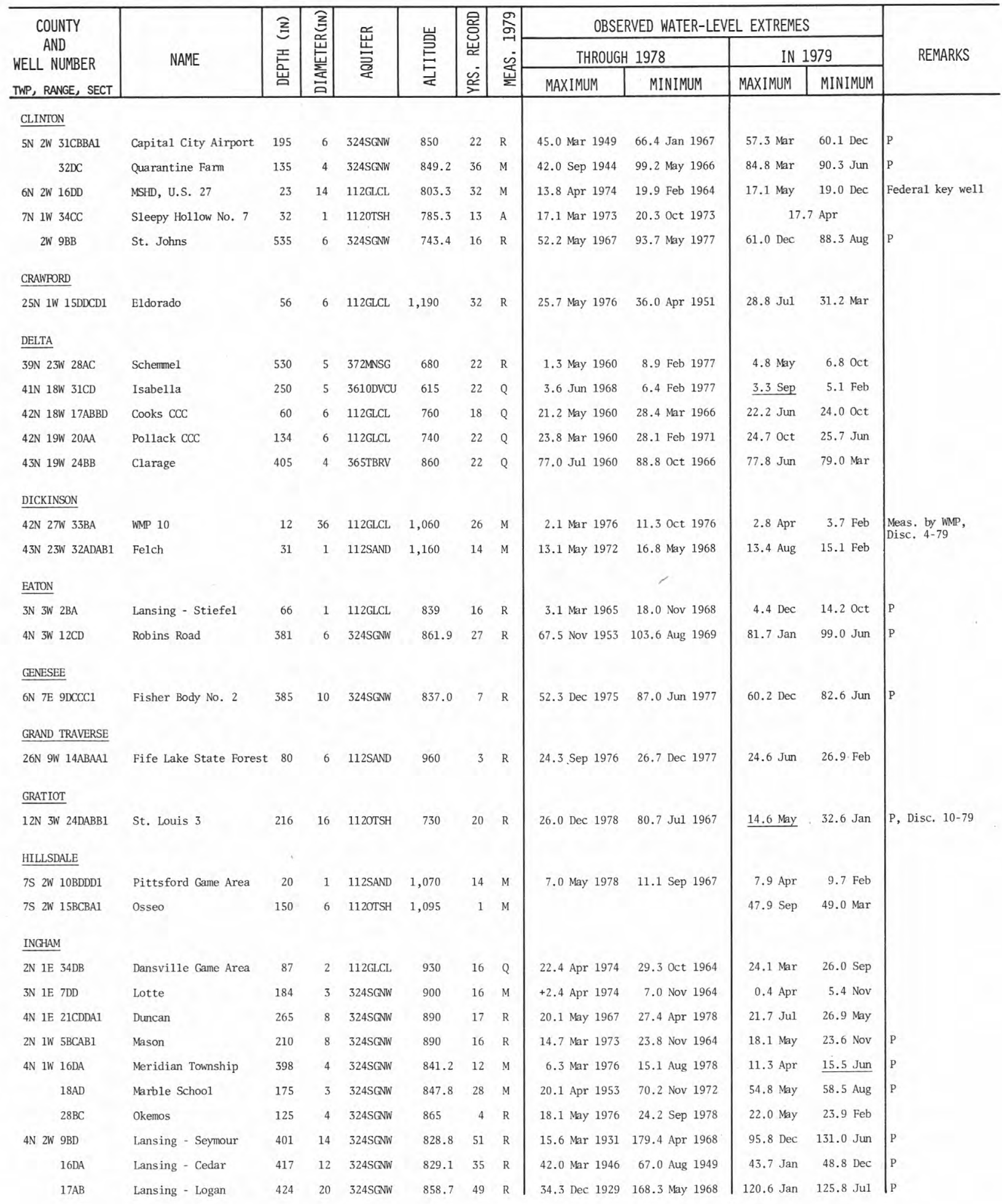


TABLE 1. RECORDS OF MICHIGAN OBSERVATION WELLS. (CONTINUED)

\begin{tabular}{|c|c|c|c|c|c|c|c|c|c|c|c|c|c|}
\hline \multirow{3}{*}{\multicolumn{2}{|c|}{$\begin{array}{c}\text { COUNTY } \\
\text { AND } \\
\text { WELL NUMBER } \\
\text { TWP, RANGE, SECT }\end{array}$}} & \multirow{3}{*}{ NAME } & \multirow{3}{*}{$\begin{array}{l}\text { ઊ } \\
\text { 产 } \\
\text { 岀 }\end{array}$} & \multirow{3}{*}{$\begin{array}{l}\text { 芯 } \\
\text { 怘 } \\
\text { 崖 } \\
\text { 完 } \\
\end{array}$} & \multirow{3}{*}{ 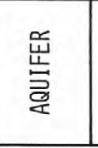 } & \multirow{3}{*}{ 菅 } & \multirow{3}{*}{ 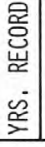 } & \multirow{3}{*}{ 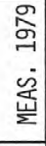 } & \multicolumn{4}{|c|}{ OBSERVED WATER-LEVEL EXTREMES } & \multirow{3}{*}{ REMARKS } \\
\hline & & & & & & & & & \multicolumn{2}{|c|}{ THROUGH 1978} & \multicolumn{2}{|c|}{ IN 1979} & \\
\hline & & & & & & & & & MAXIMUM & MINIMUM & MAXIMUM & MINIMUM & \\
\hline \multicolumn{14}{|c|}{ INGHAM (Cont' ${ }^{\prime}$ ) } \\
\hline \multirow{5}{*}{$\begin{array}{rr}4 \mathrm{~N} & 2 \mathrm{~W} \\
2 \\
2 \\
2 \\
2 \\
3 \\
2 \\
2\end{array}$} & $21 \mathrm{BA} 3$ & Lansing - Scott Park & 400 & 4 & 324 SGNW & 835 & 1 & $\mathrm{R}$ & & & $54.6 \mathrm{Jul}$ & 58.8 Jun & $P$ \\
\hline & $22 \mathrm{BC}$ & Lansing - P-5 & 338 & 12 & 324SGNW & 823.6 & 50 & M & 7.1 Jul 1932 & 80.5 Feb 1970 & 66.5 May & 78.6 Dec & $P$ \\
\hline & $24 \mathrm{CA}$ & Spartan Village & 453 & 10 & 324SGNW & 853.4 & 35 & $\mathrm{R}$ & 25.5 Mar 1946 & 105.5 May 1972 & $76.0 \mathrm{Apr}$ & 90.0 Jun & $P$ \\
\hline & $27 \mathrm{BB}$ & Fenner Arboretum & 215 & 6 & 324SGNW & 835 & 12 & $\mathrm{R}$ & $52.0 \mathrm{Jul} 1968$ & 89.5 Oct 1972 & $62.0 \mathrm{Apr}$ & $74.5 \mathrm{Sep}$ & \\
\hline & $31 \mathrm{CC}$ & Maybel Street & 204 & 3 & 324SGNN & 880.2 & 36 & M & 18.9 Apr 1952 & 45.1 Feb 1977 & $40.9 \mathrm{Nov}$ & 42.3 Mar & $P$ \\
\hline \multicolumn{14}{|l|}{$\underline{\text { IRON }}$} \\
\hline $42 \mathrm{~N} 31 \mathrm{~W}$ & W $33 \mathrm{DB}$ & WMP 7 & 10 & 1 & $112 \mathrm{GLCL}$ & 1,275 & 32 & M & +0.2 May 1960 & 6.7 Jan 1977 & $0.3 \mathrm{Apr}$ & $2.5 \mathrm{Sep}$ & Meas. by WMP \\
\hline \multirow[t]{3}{*}{$43 \mathrm{~N} 35 \mathrm{~W}$} & IW $11 \mathrm{AD}$ & WMP 23 & 47 & 36 & $112 \mathrm{GLCL}$ & 1,565 & 35 & M & 35.5 Jul 1973 & 47.1 Aug 1949 & $37.7 \mathrm{Sep}$ & $41.1 \mathrm{Apr}$ & Meas. by WMP \\
\hline & $20 \mathrm{DC}$ & WMP 25 & 48 & 1 & $112 \mathrm{GLCL}$ & 1,560 & 35 & M & 40.7 Jun 1973 & 48.3 Aug 1949 & 41.9 Aug & $44.5 \mathrm{Mar}$ & Do \\
\hline & 33BD & WMP 34 & 12 & 1 & $112 \mathrm{GLCL}$ & 1,520 & 32 & M & 1.7 Jun 1973 & 8.4 Mar 1949 & $2.2 \mathrm{Jun}$ & 6.3 Mar & Do \\
\hline \multirow{2}{*}{$44 \mathrm{~N}$} & W $10 C C$ & WMP 21 & 8 & 1 & $112 \mathrm{GLCL}$ & 1,540 & 32 & M & 2.0 Apr 1954 & 8.2 Apr 1977 & 2.2 May & 4.8 Jan & Do \\
\hline & W $14 \mathrm{BB}$ & CCC Camp & 102 & 6 & $112 \mathrm{GLCL}$ & 1,730 & 21 & Q & 91.8 Oct 1973 & 100.9 Aug 1974 & $92.4 \mathrm{Dec}$ & 93.9 May & \\
\hline $45 \mathrm{~N} 37 \mathrm{~W}$ & W $23 \mathrm{AC}$ & WMP 28 & 8 & 1 & $112 \mathrm{GLCL}$ & 1,600 & 32 & M & 0.7 Apr 1965 & 5.1 Sep 1976 & 1.2 May & $2.6 \mathrm{Sep}$ & Meas. by WMP \\
\hline $46 \mathrm{~N} 33 \mathrm{~W}$ & W $18 \mathrm{BC}$ & WMP 17 & 12 & 1 & 112GLCL & 1,560 & 32 & M & 2.8 Apr 1949 & dry Feb 1956 & 4.7 Jun & $7.6 \mathrm{Feb}$ & Do \\
\hline \multicolumn{14}{|l|}{ JACKSON } \\
\hline $3 S 1 W 2$ & 2BDBAI & Jackson - Hamburg & 400 & 12 & $\begin{array}{l}\text { 324SGNW, } \\
\text { 337MRSL }\end{array}$ & 935 & 14 & $\mathrm{R}$ & 16.3 Jan 1971 & 68.8 Jun 1971 & $25.7 \mathrm{Nov}$ & $47.3 \mathrm{Jul}$ & $P$ \\
\hline 1 & $10 \mathrm{DC}$ & Summit Township & 323 & 12 & $\begin{array}{l}\text { 324SGNW, } \\
\text { 337MRSL }\end{array}$ & 935 & 20 & $\mathrm{R}$ & 14.3 Jan 1961 & 36.5 Jun 1971 & $18.6 \mathrm{Dec}$ & $32.4 \mathrm{Jul}$ & $P$ \\
\hline & 11AAl & Jackson - 4a Belden & 360 & 6 & $\begin{array}{l}\text { 324SGNW, } \\
\text { 337MRSL }\end{array}$ & 935 & 22 & $\mathrm{D}$ & 18.6 Jan 1961 & 119.1 Jun 1971 & $48.6 \mathrm{Dec}$ & $110.8 \mathrm{Jun}$ & $P$, Meas, by owner \\
\hline & 11AADD2 & Jackson - Belden & 36 & 3 & $1120 \mathrm{TSH}$ & 928.8 & 18 & $\mathrm{R}$ & +1.9 Apr 1977 & 18.2 Nov 1964 & $+0.5 \mathrm{Mar}$ & $3.6 \mathrm{Sep}$ & \\
\hline \multicolumn{14}{|c|}{$\underline{\text { KALAMAZOO }}$} \\
\hline \multirow{11}{*}{$\begin{array}{l}\text { 2S } 10 \mathrm{~W} \\
11 \mathrm{~W}\end{array}$} & $4 \mathrm{D}$ & Kalamazoo - Campbe11 & 13 & 4 & 1120TSH & 836.5 & 11 & M & 1.9 Apr 1974 & 3.7 Aug 1977 & $1.6 \mathrm{Ju} 1$ & $3.5 \mathrm{Dec}$ & $\mathrm{P}$ \\
\hline & $9 \mathrm{~B}$ & Kalamazoo - Schoonover & 21 & 6 & 1120TSH & 828 & 11 & $\mathrm{R}$ & +1.0 Apr 1975 & 0.4 Sep 1971 & +0.6 Jun & $1.5 \mathrm{Dec}$ & $\mathrm{P}$ \\
\hline & $3 \mathrm{AA}$ & Brown 61 & 36 & 6 & $1120 \mathrm{TSH}$ & 763.2 & 24 & $\mathrm{R}$ & 7.7 Mar 1974 & 14.0 Aug 1967 & $9.4 \mathrm{Dec}$ & 11.5 Oct & P, Disc. $12-79$ \\
\hline & $10 \mathrm{DB}$ & General Printing Ink & 49 & 10 & $1120 \mathrm{TSH}$ & 761 & 12 & $\mathrm{R}$ & 11.1 Ju1 1978 & 23.6 Oct 1978 & $12.7 \mathrm{Mar}$ & 18.9 Aug & P, Disc. $12-79$ \\
\hline & $14 \mathrm{DC}$ & Brown 13 & 90 & 12 & $112 \mathrm{OTSH}$ & 780 & 8 & $\mathrm{R}$ & 10.2 Jan 1973 & 17.9 Feb 1975 & $12.1 \mathrm{Apr}$ & $16.9 \mathrm{Feb}$ & P, Disc. $12-79$ \\
\hline & 20BB2 & Kalamazoo - Kenda11 & 106 & 4 & $1120 \mathrm{TSH}$ & 880 & 12 & $\mathrm{R}$ & $12.5 \mathrm{Feb} 1976$ & 48.4 Jun 1971 & $17.9 \mathrm{Dec}$ & $42.8 \mathrm{Ju} 1$ & $P$ \\
\hline & $22 \mathrm{CD}$ & Ka1amazoo-Stockbridge & 137 & 4 & $1120 \mathrm{TSH}$ & 764.7 & 20 & $\mathrm{R}$ & 4.8 Feb 1975 & 31.1 Aug 1961 & 8.3 May & 9.3 Oct & $\mathrm{P}$ \\
\hline & $23 \mathrm{AD}$ & Allied Paper & 43 & 12 & $1120 \mathrm{TSH}$ & 760 & 12 & $\mathrm{R}$ & +0.4 Apr 1975 & 22.0 Feb 1970 & 0.9 Mar & 8.5 Sep & P, Disc. $12-79$ \\
\hline & 28AA & Kalamazoo - Maple & 245 & 4 & 1120TSH & 820 & 11 & $\mathrm{R}$ & 35.5 Dec 1978 & 61.6 Jun 1973 & $32.9 \mathrm{Jan}$ & 49.7 May & $\mathrm{P}$ \\
\hline & $31 \mathrm{CD}$ & Kalamazoo - Colony & 226 & 4 & $1120 \mathrm{TSH}$ & 910 & 11 & $\mathrm{R}$ & 51.1 Jun 1974 & 71.8 May 1978 & $51.9 \mathrm{Nov}$ & 67.1 Jun & $\mathrm{P}$ \\
\hline & $36 \mathrm{CB}$ & Kalamazoo - Emera1d & 226 & 4 & $1120 \mathrm{TSH}$ & 860 & 11 & $\mathrm{R}$ & 25.7 May 1976 & 50.4 Jun 1971 & 26.4 May & $42.6 \mathrm{Jul}$ & $\mathrm{P}$ \\
\hline \multirow[t]{7}{*}{ 3S $11 W$} & $4 A D 1$ & Kalamazoo - A-D & 135 & 3 & $112 \mathrm{OTSH}$ & 854.0 & 21 & $\mathrm{R}$ & 0.5 May 1967 & 12.9 Ju1 1964 & 1.5 Mar & $9.5 \mathrm{Jul}$ & $\mathrm{P}$ \\
\hline & $4 A D 2$ & Kalamazoo - A-S & 40 & 3 & $1120 \mathrm{TSH}$ & 854.0 & 21 & $\mathrm{R}$ & +0.2 Sep 1975 & 9.1 Nov 1959 & 0.4 Jun & $2.6 \mathrm{Ju} 1$ & $\mathrm{P}$ \\
\hline & $14 \mathrm{AA}$ & Upjohn 28 & 233 & 16 & $1120 \mathrm{TSH}$ & 870 & 13 & $\mathrm{R}$ & 25.2 Feb 1976 & 45.2 Ju1 1977 & $26.4 \mathrm{Apr}$ & $39.4 \mathrm{Jul}$ & $P$ \\
\hline & $22 \mathrm{BD}$ & Portage - Site C & 120 & 8 & $1120 \mathrm{TSH}$ & 865 & 13 & $\mathrm{R}$ & 5.9 Jun. 1967 & 10.2 Nov 1976 & 7.0 May & $8.8 \mathrm{Nov}$ & $\mathrm{P}$ \\
\hline & $11 \mathrm{BD}$ & Kalamazoo - Atwater & 248 & 3 & 1120TSH & 880 & 19 & $\mathrm{R}$ & +3.0 Sep 1969 & 1.0 Aug 1977 & +1.1 May & $0.0 \mathrm{Apr}$ & P. \\
\hline & $11 \mathrm{AD} 1$ & Ka1amazoo - Sabo-D & 300 & 4 & 1120TSH & 877 & 7 & $\mathrm{R}$ & 4.5 Jul 1973 & 16.4 Ju1 1977 & 5.9 Mar & $16.3 \mathrm{Apr}$ & $\mathrm{P}$ \\
\hline & 11AD2 & Kalamazoo - Sabo-S & 38 & 6 & 112OTSH & 877 & 7 & $\mathrm{R}$ & 9.1 Aug 1975 & 12.7 Aug 1977 & $9.9 \mathrm{Mar}$ & 11.3 May & $\mathrm{P}$ \\
\hline 4S $11 \mathrm{~W}$ & $3 \mathrm{CDDA} 1$ & Prairie View Park & 190 & 4 & 112OTSH & 870 & 11 & $\mathrm{R}$ & 18.3 Jun 1973 & 20.5 Dec 1977 & $18.2 \mathrm{Apr}$ & 19.7 Nov & \\
\hline
\end{tabular}


TABLE 1. RECORDS OF MICHIGAN OBSERVATION WELLS. (CONTINUED)

\begin{tabular}{|c|c|c|c|c|c|c|c|c|c|c|c|c|}
\hline \multirow{3}{*}{$\begin{array}{c}\text { COUNTY } \\
\text { AND } \\
\text { WELL NUMBER } \\
\text { TWP, RANGE, SECT }\end{array}$} & \multirow{3}{*}{ NAME } & \multirow{3}{*}{$\begin{array}{l}\text { そ } \\
\text { 工 } \\
\text { 㟧 }\end{array}$} & \multirow{3}{*}{ 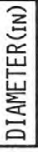 } & \multirow{3}{*}{$\begin{array}{l}\text { 岀 } \\
\text { 容 } \\
\text { 宅 }\end{array}$} & \multirow{3}{*}{ 菅 } & \multirow{3}{*}{ 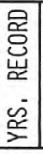 } & \multirow{3}{*}{ 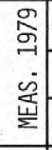 } & \multicolumn{4}{|c|}{ OBSERVED WATER-LEVEL EXTREMES } & \multirow{3}{*}{ REMARKS } \\
\hline & & & & & & & & \multicolumn{2}{|c|}{ THROUGH 1978} & \multicolumn{2}{|c|}{ IN 1979} & \\
\hline & & & & & & & & MAXIMUM & MINIMUM & MAXIMUM & MINIMUM & \\
\hline \multicolumn{13}{|l|}{$\underline{\text { KENT }}$} \\
\hline $5 \mathrm{~N} 12 \mathrm{~W} 4 \mathrm{DCCD} 1$ & Wyoming - Wobma & 86 & 6 & 112GRVL & 868.0 & 18 & $\mathrm{R}$ & 7.8 Oct 1978 & 12.9 Aug 1964 & 8.7 Mar & $11.0 \mathrm{Sep}$ & \\
\hline $6 \mathrm{~N}$ 10W $30 \mathrm{AA}$ & Kent County Airport & 184 & 10 & 112GLCL & 800 & 14 & $\mathrm{R}$ & 83.1 Apr 1977 & 108.0 Sep 1967 & 83.4 Mar & $100.4 \mathrm{Jul}$ & $\mathrm{P}$ \\
\hline $12 \mathrm{~W} 17 \mathrm{AD} 1$ & Alloytek & 30 & 12 & $112 \mathrm{GLCL}$ & 606 & 30 & M & 6.8 Apr 1965 & 16.4 Feb 1954 & 7.2 Apr & $11.8 \mathrm{Jan}$ & P, Meas . by owner \\
\hline $17 \mathrm{AD} 2$ & Alloytek & 26 & 6 & 112GLCL & 606.0 & 30 & M & 6.8 Apr 1965 & 16.3 Feb 1954 & $9.0 \mathrm{Jun}$ & $12.0 \mathrm{Jan}$ & P, Meas . by owner \\
\hline $\begin{array}{l}\text { 10N 12W 13DD } \\
\text { LENAWEE }\end{array}$ & Rogue River Game Area & 30 & 1 & 112GLCL & 785 & 14 & Q & 0.8 Jan 1975 & 9.2 Oct 1969 & 2.1 May & $7.9 \mathrm{Sep}$ & \\
\hline $5 S \quad 1 E$ 12DD & Onsted Game Area & 39 & 1 & $112 \mathrm{GLCL}$ & 1,000 & 14 & M & 16.1 May 1975 & 19.3 Sep 1971 & 16.2 Apr & 17.8 oct & \\
\hline $\begin{array}{l}\text { 6S 4E 8DDBA1 } \\
\text { LIVINGSTON }\end{array}$ & Fisher Body & 81 & 8 & 1120TSH & 800 & 15 & $\mathrm{R}$ & 11.2 May 1976 & 18.4 Feb 1965 & $13.5 \mathrm{Jul}$ & $14.8 \mathrm{Feb}$ & $\mathrm{P}$ \\
\hline $1 \mathrm{~N} 6 \mathrm{E}$ 13DBAB1 & American Aggregate & 29 & 2 & $1120 \mathrm{TSH}$ & 930 & 10 & $\mathrm{R}$ & 12.1 Apr 1974 & 18.1 Feb 1972 & 15.6 May & $21.60 \mathrm{ct}$ & \\
\hline $\begin{array}{l}\text { 2N 6E 31BA2 } \\
\text { MACKINAC }\end{array}$ & Brighton & 83 & 10 & 112GLCL & 935 & 6 & $\mathrm{R}$ & 27.2 Sep 1975 & $58.6 \mathrm{Jul} 1978$ & $31.8 \mathrm{Apr}$ & 54.5 Jun & P \\
\hline $41 \mathrm{~N} 5 \mathrm{~W} 23 \mathrm{BC}$ & Round Lake CCC & 47 & 6 & 355SLINH & 610 & 24 & $Q$ & 4.3 May 1959 & 17.5 Mar 1959 & $7.5 \mathrm{Mar}$ & 12.1 Aug & \\
\hline $\begin{array}{l}\text { 42N 2W 7AABB1 } \\
\text { MARQUETTE }\end{array}$ & Pontchartrain CCC & 102 & 6 & $355 \mathrm{MNSQ}$ & 680 & 24 & $\mathrm{R}$ & 13.1 May 1960 & 32.3 Feb 1977 & $14.1 \mathrm{Apr}$ & $28.0 \mathrm{Feb}$ & \\
\hline $46 \mathrm{~N} 25 \mathrm{~W} 16 \mathrm{DD}$ & Sands Station & 48 & 1 & 112GLCL & $1,198.4$ & 17 & M & 27.1 Jul 1969 & 37.7 May 1964 & 27.5 Sep & 31.7 Apr & \\
\hline $47 \mathrm{~N} 25 \mathrm{~W} 19 \mathrm{CC}$ & Cascade Junction & 86 & 1 & 112GLCL & $1,222.1$ & 17 & M & 25.0 Jul 1973 & 39.0 Feb 1965 & 26.0 May & 28.9 Jan & Disc. $5-79$ \\
\hline $20 \mathrm{CC}$ & East Cascade Junction & 103 & 1 & 112GLCL & $1,229.8$ & 17 & M & 78.5 Oct 1973 & 90.6 Jun 1965 & $79.4 \mathrm{Dec}$ & $81.8 \mathrm{Apr}$ & \\
\hline $32 \mathrm{CA}$ & Gentian & 122 & 1 & 112GLCL & $1,239.2$ & 15 & M & 83.6 Apr 1976 & 100.0 Oct 1964 & $91.1 \mathrm{Dec}$ & 92.7 Jun & \\
\hline $26 \mathrm{~W} 27 \mathrm{BC}$ & Laitala & 31 & 1 & 112GLCL & 1,290 & 12 & M & +1.8 May 1978 & 10.1 oct 1969 & +3.4 Apr & 4.6 oct & \\
\hline 36BBDB1 & C.C. - Goose Lake & 56 & 8 & 1120TSH & 1,210 & 15 & $\mathrm{R}$ & 3.6 Apr 1969 & 8.8 Mar 1977 & 4.7 Apr & $6.3 \mathrm{Feb}$ & \\
\hline 28W 3CCDC1 & Ely Township & 75 & 8 & 112OTSH & $1,572.0$ & 19 & $\mathrm{R}$ & 9.7 May 1973 & 19.3 Apr 1964 & 10.5 Jun & $15.2 \mathrm{Mar}$ & Federal key well \\
\hline $48 \mathrm{~N} 26 \mathrm{~W} 34 \mathrm{DA}$ & Eag1e Mi11s & 31 & 1 & 112GLCL & $1,279.6$ & 17 & M & 2.0 Apr 1967 & 7.0 Apr 1964 & $2.6 \mathrm{Apr}$ & $4.8 \mathrm{Jan}$ & \\
\hline $29 \mathrm{~W} 30 \mathrm{CC}$ & Van Riper Park & 78 & 6 & 112GLCL & 1,560 & 11 & M & 9.6 May 1973 & 15.7 Feb 1977 & 10.7 Jun & 13.6 Feb & \\
\hline $\begin{array}{l}\text { 49N } 30 \mathrm{~W} 22 \mathrm{AC} \\
\text { MENOMINEE }\end{array}$ & WMP 13 & 17 & 1 & 112GLCL & 1,680 & 32 & M & 0.6 May 1951 & $13.3 \mathrm{Sep} 1948$ & $8.2 \mathrm{Apr}$ & $10.1 \mathrm{Sep}$ & Meas. b y WMP \\
\hline $\begin{array}{l}\text { 37N 26W 19DADAl } \\
\text { MDNROE }\end{array}$ & Carney & 17 & 4 & 365TBRV & 800 & 21 & M & 3.6 May 1973 & 8.6 Jan 1977 & $3.5 \mathrm{Apr}$ & $5.6 \mathrm{Sep}$ & \\
\hline $7 \mathrm{~S}$ 6E $15 \mathrm{ADBB} 1$ & Petersburg Game Area & 17 & 1 & $112 \mathrm{GLCL}$ & 675 & 14 & M & $3.0 \mathrm{Feb} 1966$ & $6.8 \mathrm{Nov} 1978$ & 5.2 May & $6.5 \mathrm{Mar}$ & \\
\hline $\begin{array}{l}\text { 7S 6E 15ACAA1 } \\
\text { MUSKEGON }\end{array}$ & Petersburg - Rock & 73 & 6 & 348DRRV & 680 & 1 & M & & & $35.9 \mathrm{Apr}$ & $40.2 \mathrm{Nov}$ & \\
\hline $\begin{array}{l}\text { 11N 15W 34ADDD1 } \\
\text { OAKL_AND }\end{array}$ & Muskegon Game Area & 31 & 1 & $112 \mathrm{SAND}$ & 595 & 14 & Q & +0.2 Apr 1978 & 4.7 Sep 1972 & 1.0 May & $3.3 \mathrm{Sep}$ & \\
\hline $2 \mathrm{~N} 7 \mathrm{E} 5 \mathrm{BA}$ & Honeywell Lake Road & 44 & 2 & $112 \mathrm{GLCL}$ & 1,020 & 12 & $\mathrm{R}$ & 23.9 Apr 1976 & $28.9 \mathrm{Dec} 1971$ & 25.8 Aug & $28.2 \mathrm{Mar}$ & \\
\hline 8E $18 D B A D 1$ & Proud Lake Park & 45 & 6 & $1120 \mathrm{TSH}$ & 910 & 11 & $\mathrm{R}$ & 2.8 May 1974 & 6.4 Sep 1971 & 4.2 May & $5.8 \mathrm{Jan}$ & P \\
\hline
\end{tabular}


TABLE 1. RECORDS OF MICHIGAN OBSERVATION WELLS. (CONTINUED)

\begin{tabular}{|c|c|c|c|c|c|c|c|c|c|c|c|c|}
\hline \multirow{3}{*}{$\begin{array}{c}\text { COUNTY } \\
\text { AND } \\
\text { WELL NUMBER } \\
\text { TWP, RANGE, SECT } \\
\end{array}$} & \multirow{3}{*}{ NAME } & \multirow{3}{*}{$\begin{array}{l}\Xi \\
\Xi \\
\text { 志 } \\
\text { 㟧 }\end{array}$} & \multirow{3}{*}{ 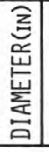 } & \multirow{3}{*}{$\begin{array}{l}\text { 品 } \\
\text { 莺 } \\
\text { 足 }\end{array}$} & \multirow{3}{*}{$\stackrel{\text { 㟔 }}{\text { 点 }}$} & \multirow{3}{*}{ 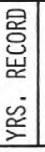 } & \multirow{3}{*}{ 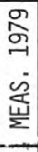 } & \multicolumn{4}{|c|}{ OBSERVED WATER-LEVEL EXTREMES } & \multirow{3}{*}{ REMARKS } \\
\hline & & & & & & & & \multicolumn{2}{|c|}{ THROUGH 1978} & \multicolumn{2}{|c|}{ IN 1979} & \\
\hline & & & & & & & & MAXIMUM & MINIMUM & MAXIMUM & MINIMUM & \\
\hline \multicolumn{13}{|l|}{ OAKLAND (Cont'd) } \\
\hline $3 \mathrm{~N} 7 \mathrm{E} 5 \mathrm{DA}$ & Fish Lake Road & 49 & 2 & 112GLCL & 1,055 & 11 & $\mathrm{R}$ & 29.5 Jun 1976 & 38.7 Dec 1972 & 35.9 Jun & $36.8 \mathrm{Dec}$ & \\
\hline 8E $3 \mathrm{DBAB} 1$ & White Lake Road & 163 & 6 & 1120TSH & 1,000 & 8 & $\mathrm{R}$ & 7.2 May 1976 & 11.2 Sep 1978 & 9.0 May & 10.7 Feb & \\
\hline $10 \mathrm{AB}$ & Teggerdine Road & 163 & 6 & 112 GLCL & 1,000 & 8 & $\mathrm{R}$ & 27.8 Mar 1976 & 30.8 Sep 1978 & 28.9 May & $30.3 \mathrm{Feb}$ & \\
\hline $10 \mathrm{E} 13 \mathrm{AC}$ & Oakland University & 183 & 6 & 112GLCL & 940 & 19 & $\mathrm{R}$ & 56.9 Feb 1978 & 93.5 Ju1 1963 & $57.2 \mathrm{Apr}$ & $58.4 \mathrm{Dec}$ & \\
\hline $5 \mathrm{~N} 8 \mathrm{E} \quad 8 \mathrm{AC}$ & Holly Recreation Area & 42 & 1 & $112 \mathrm{GLCL}$ & 930 & 14 & M & 22.3 Apr 1974 & 26.5 Sep 1966 & $24.9 \mathrm{Apr}$ & 26.2 oct & \\
\hline \multicolumn{13}{|l|}{ OCEANA } \\
\hline 13N 15W 18AAAA1 & Hesperia & 79 & 6 & $1120 \mathrm{TSH}$ & 703 & 2 & $\mathrm{R}$ & 39.0 Jun 1978 & 40.4 Apr 1978 & 36.6 Jun & $40.2 \mathrm{Mar}$ & \\
\hline \multicolumn{13}{|l|}{ OGEMAW } \\
\hline 23N 1E 2 BAAA1 & Rose City Road-D & 105 & 1 & 112GLCL & 1,265 & 12 & $Q$ & 73.6 Oct 1976 & 78.2 Apr 1969 & $75.4 \mathrm{Jan}$ & $76.7 \mathrm{Apr}$ & \\
\hline 2BAAA2 & Rose City Road-S & 20 & 1 & 112 SAND & 1,265 & 12 & Q & 7.6 Apr 1976 & 13.6 Dec 1972 & 9.7 Apr & 11.2 Oct & \\
\hline \multicolumn{13}{|l|}{ ONTONAGON } \\
\hline $46 \mathrm{~N} 38 \mathrm{~W}$ 30ADDD1 & USFS & 65 & 1 & 112 SDGV & 1,530 & 13 & M & 16.0 Jun 1973 & 19.7 Mar 1978 & 16.0 Jun & $18.3 \mathrm{Mar}$ & \\
\hline $51 \mathrm{~N} 41 \mathrm{~W} 8 \mathrm{BDBC1}$ & Silver City & 100 & 6 & 420FRED & 620 & 22 & M & 8.2 Apr 1959 & 21.8 Dec 1976 & 9.4 May & $16.1 \mathrm{Sep}$ & \\
\hline \multicolumn{13}{|l|}{ OTSEGO } \\
\hline $30 \mathrm{~N} 3 W \quad 19 A B B B 1$ & Gaylord & 90 & 6 & 112OTSH & 1,308 & 1 & M & & & $30.7 \mathrm{Jul}$ & 33.9 Jun & \\
\hline \multicolumn{13}{|l|}{ PRESQUE ISLE } \\
\hline $33 \mathrm{~N}$ 6E $8 \mathrm{BBBB} 1$ & Styma & 61 & 6 & 341TRVR & 800 & 21 & Q & 5.4 Apr 1967 & 18.8 Mar 1963 & $5.1 \mathrm{Mar}$ & $14.20 c t$ & \\
\hline \multicolumn{13}{|l|}{ ROSCOMDN } \\
\hline $24 \mathrm{~N} 2 \mathrm{~W} 20 \mathrm{BABA} 1$ & Exp. Station & 14 & 8 & $1120 \mathrm{TSH}$ & $1,145.3$ & 46 & $\mathrm{R}$ & 2.1 Apr 1976 & 6.2 Dec 1949 & 3.1 May & $5.2 \mathrm{Feb}$ & Federal key we11 \\
\hline \multicolumn{13}{|l|}{ SAGINAW } \\
\hline 10N 1E 22DADA1 & Marion Springs & 210 & 6 & 324SGNW & 657 & 2 & $\mathrm{R}$ & 8.8 Apr 1978 & 10.0 Sep 1978 & 8.8 May & 9.8 Sep & \\
\hline \multicolumn{13}{|l|}{ SANILAC } \\
\hline 13N 13E 12ADAA1 & Minden Game Area & 130 & 6 & 337MRSL & 805 & 3 & $\mathrm{R}$ & 17.7 Apr 1978 & 22.3 Sep 1978 & 18.3 May & $22.70 \mathrm{ct}$ & \\
\hline \multicolumn{13}{|l|}{ SCHOOLCRAFT } \\
\hline $45 \mathrm{~N}$ 13W $16 \mathrm{CCCB} 1$ & Seney & 154 & 4 & 3610DVCU & 710 & 28 & $\mathrm{R}$ & 4.6 Apr 1971 & 6.5 oct 1963 & 4.7 Apr & 5.5 Jul & \\
\hline $47 \mathrm{~N} \quad 16 \mathrm{~W} \quad 30 \mathrm{BBBB} 1$ & Cusino CCC & 57 & 6 & 368PRDC & 900 & 23 & $\mathrm{R}$ & 5.7 May 1960 & 16.4 Feb 1977 & $6.0 \mathrm{Apr}$ & $14.30 c t$ & \\
\hline VAN BUREN & & & & & & & & & & & & \\
\hline $3 \mathrm{~S} 14 \mathrm{~W} 6 \mathrm{BA}$ & Martin & 59 & 1 & 112GLCL & 740 & 17 & M & 37.2 May 1974 & 43.3 Nov 1964 & 37.4 Apr & $39.8 \mathrm{Jan}$ & \\
\hline WASHTENAW & & & & & & & & & & & & \\
\hline $2 S$ 3E $9 D A A B 2$ & Waterloo Park & 48 & 6 & 112 SDGV & 970 & 11 & $\mathrm{R}$ & 4.1 May 1974 & 7.0 Aug 1971 & 4.7 Apr & $6.7 \mathrm{Ju1}$ & $\mathrm{P}$ \\
\hline $3 \mathrm{~S} 6 \mathrm{E} \quad 16 \mathrm{BCCD} 1$ & Ann Arbor & 55 & 10 & 112GLCL & 821.5 & 17 & $\mathrm{R}$ & 0.7 Mar 1974 & 15.9 Oct 1964 & 1.0 May & 4.9 oct & $\mathrm{P}$ \\
\hline $7 \mathrm{E} 5 \mathrm{BB}$ & Ypsilanti - Superior & 69 & 8 & 112GLCL & 720 & 18 & $\mathrm{R}$ & 1.8 Feb 1965 & $21.4 \mathrm{Dec} 1965$ & $3.3 \mathrm{Nov}$ & $10.0 \mathrm{Feb}$ & $\mathrm{P}$ \\
\hline $9 \mathrm{AD}$ & Ypsilanti - Gilvert & 94 & 6 & 112GLCL & 710 & 29 & $\mathrm{R}$ & 29.1 Nov 1945 & 78.8 Oct 1974 & $66.2 \mathrm{Nov}$ & 72.5 Aug & $\mathrm{P}$ \\
\hline 24CA1 & Ypsilanti Township 104 & 87 & 4 & 112GLCL & 665.6 & 34 & $\mathrm{R}$ & 5.8 Jan 1950 & 22.7 Feb 1971 & 12.5 May & $15.8 \mathrm{Nov}$ & $\mathrm{P}$ \\
\hline $24 \mathrm{CD}$ & Ypsilanti Township 117 & 75 & 6 & 112GLCL & 657.8 & 33 & $\mathrm{R}$ & 5.3 May 1978 & 63.2 Feb 1970 & 6.0 May & $18.9 \mathrm{Nov}$ & $\mathrm{P}$ \\
\hline WEXFORD & & & & & & & & & & & & 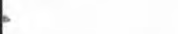 \\
\hline $22 \mathrm{~N} 12 \mathrm{~W} 13 \mathrm{BA}$ & Harrietta Fish Hatchery & y 141 & 4 & 112GLCL & 1,060 & 19 & $\mathrm{R}$ & $13.8 \operatorname{Mar} 1970$ & +1.5 Jan 1966 & $+8.5 \mathrm{Jan}$ & $+0.40 \mathrm{ct}$ & $\mathrm{P}$ \\
\hline
\end{tabular}


TABLE 2. REPORTED GROUND-WATER PUMPAGE, IN 1979. (IN MILLIONS OF GALLONS)

\begin{tabular}{|c|c|c|c|c|c|c|c|c|c|c|c|c|c|c|c|}
\hline $\begin{array}{c}\text { COUNTY } \\
\text { AND } \\
\text { WATER USER }\end{array}$ & JAN & FEB & MAR & APR & MAY & JUN & JUL & AUG & SEP & OCT & NOV & $\mathrm{DEC}$ & $\begin{array}{l}1979 \\
\text { TOTAL }\end{array}$ & $\begin{array}{l}\text { MAX } \\
\text { DAY }\end{array}$ & $\begin{array}{l}\text { MIN } \\
\text { DAY }\end{array}$ \\
\hline $\begin{array}{l}\text { ALCONA } \\
\text { Harrisville }\end{array}$ & 1.6 & .8 & 1.8 & 1.2 & 1.3 & 1.7 & 2.4 & 2.1 & 1.8 & 1.6 & 1.2 & 1.3 & 18.8 & .101 & .020 \\
\hline \multicolumn{16}{|l|}{ ALGER } \\
\hline $\begin{array}{l}\text { Burt Twp. } \\
\text { Chatham }\end{array}$ & $\begin{array}{l}4.5 \\
1.0\end{array}$ & $\begin{array}{r}3.5 \\
.9\end{array}$ & $\begin{array}{l}3.3 \\
1.0\end{array}$ & $\begin{array}{r}4.5 \\
.8\end{array}$ & $\begin{array}{r}1.1 \\
.8\end{array}$ & $\begin{array}{r}2.5 \\
.7\end{array}$ & $\begin{array}{r}5.4 \\
.9\end{array}$ & $\begin{array}{r}12.5 \\
.8\end{array}$ & $\begin{array}{r}11.0 \\
.8\end{array}$ & $\begin{array}{r}2.4 \\
.8\end{array}$ & $\begin{array}{r}1.4 \\
.9\end{array}$ & $\begin{array}{l}1.7 \\
1.0\end{array}$ & $\begin{array}{l}53.8 \\
10.4\end{array}$ & $\begin{array}{l}-- \\
-\end{array}$ & $\begin{array}{l}-\cdot \\
-\end{array}$ \\
\hline \multicolumn{16}{|l|}{ ALLEGAN } \\
\hline Allegan & 27.5 & 27.5 & 32.2 & 29.1 & 35.4 & 40.9 & 43.1 & 34.0 & 33.5 & 28.9 & 26.2 & 29.5 & 387.8 & 1.911 & .524 \\
\hline $\begin{array}{l}\text { Douglas } \\
\text { Otsego }\end{array}$ & $\begin{array}{r}4.9 \\
34.6\end{array}$ & $\begin{array}{r}4.8 \\
32.6\end{array}$ & $\begin{array}{r}4.9 \\
35.7\end{array}$ & $\begin{array}{r}3.4 \\
33.7\end{array}$ & $\begin{array}{r}4.8 \\
36.6\end{array}$ & $\begin{array}{r}7.7 \\
36.8\end{array}$ & $\begin{array}{r}8.0 \\
39.6\end{array}$ & $\begin{array}{r}6.2 \\
35.2\end{array}$ & $\begin{array}{r}5.1 \\
34.0\end{array}$ & $\begin{array}{r}4.3 \\
34.8\end{array}$ & $\begin{array}{r}3.6 \\
30.6\end{array}$ & $\begin{array}{r}3.7 \\
31.1\end{array}$ & $\begin{array}{r}61.4 \\
415.3\end{array}$ & $1 .-\overline{669}$ &.$\overline{800}$ \\
\hline Plainwel1 & 12.8 & 13.6 & 13.3 & 13.6 & 16.0 & 17.1 & 20.4 & 14.9 & 16.2 & 14.0 & 12.8 & 13.1 & 177.8 & 1.043 & .213 \\
\hline Saugatuck & 8.0 & 6.2 & 6.7 & 8.6 & 8.7 & 8.8 & 10.8 & 9.5 & 8.0 & 6.2 & 5.9 & 6.5 & 93.9 & -- & -- \\
\hline \multicolumn{16}{|l|}{ ANTRIM } \\
\hline Bellaire & 2.3 & 2.4 & 2.0 & 2.1 & 2.3 & 3.1 & 4.4 & 3.1 & 2.7 & 2.7 & 2.6 & 2.8 & 32.5 & $z-$ & $\because-$ \\
\hline $\begin{array}{l}\text { Central Lake } \\
\text { E11sworth }\end{array}$ & $\begin{array}{l}3.5 \\
1.5\end{array}$ & $\begin{array}{l}3.5 \\
1.5\end{array}$ & $\begin{array}{l}4.1 \\
1.4\end{array} \quad-4$ & $\begin{array}{l}4.2 \\
1.1\end{array}$ & $\begin{array}{l}6.2 \\
1.1\end{array}$ & $\begin{array}{l}4.2 \\
1.7\end{array}$ & $\begin{array}{l}5.0 \\
2.0\end{array}$ & $\begin{array}{l}3.6 \\
1.6\end{array}$ & $\begin{array}{l}3.7 \\
1.6\end{array}$ & $\begin{array}{l}2.9 \\
1.5\end{array}$ & $\begin{array}{r}3.2 \\
.8\end{array}$ & $\begin{array}{r}2.8 \\
.9\end{array}$ & $\begin{array}{l}46.9 \\
16.7\end{array}$ & .305 & .081 \\
\hline Mancelona & 13.1 & 13.2 & 15.6 & 11.5 & 11.8 & 12.2 & 15.8 & 12.9 & 14.4 & 11.7 & 8.9 & 8.0 & 149.1 & -- & -- \\
\hline \multicolumn{16}{|l|}{ BARRY } \\
\hline Hastings & 22.6 & 21.6 & 23.9 & 20.4 & 23.9 & 27.8 & 30.8 & 24.4 & 23.4 & 24.6 & 19.8 & 20.5 & 283.7 & 1.870 & .476 \\
\hline $\begin{array}{l}\text { Midd1eville } \\
\text { Nashville }\end{array}$ & 15.4 & $\begin{array}{r}12.8 \\
3.4\end{array}$ & $\begin{array}{r}13.0 \\
3.9\end{array}$ & 11.6 & 14.4 & $\begin{array}{r}15.0 \\
3.9\end{array}$ & 15.1 & 13.1 & 13.4 & 12.8 & 11.1 & 12.1 & 159.8 & -- & $-\overline{0} 6$ \\
\hline & & & & & & & & & & & & & & & \\
\hline \multicolumn{16}{|l|}{ BENZIE } \\
\hline Beulah & 1.3 & 2.2 & 1.0 & 1.3 & 2.8 & 3.1 & 5.2 & 4.3 & 2.6 & 2.8 & 2.3 & 2.3 & 31.2 & .586 & -- \\
\hline Frankfort & 8.0 & 7.0 & 7.5 & 8.2 & 9.0 & 12.0 & 15.5 & 16.0 & 10.0 & 10.0 & 9.5 & 8.0 & e) 120.7 & -- & -- \\
\hline \multicolumn{16}{|l|}{ BERRIEN } \\
\hline Berrien Springs & 14.8 & 14.6 & 13.8 & 11.9 & 15.2 & 19.8 & $21: 2$ & 13.1 & 16.2 & 13.1 & 11.7 & 11.4 & 176.8 & -- & -- \\
\hline Buchanan & 45.0 & 42.0 & 48.0 & 50.2 & 55.5 & 60.0 & 66.2 & 69.1 & 69.7 & 71.6 & 67.7 & 49.0 & 694.0 & 2.904 & 1.270 \\
\hline Coloma & 12.9 & 11.5 & 11.0 & 11.4 & 11.8 & 12.9 & 13.9 & 13.1 & 13.0 & 22.2 & 17.8 & 13.2 & 164.7 & .916 & .258 \\
\hline Niles & 76.0 & 74.1 & 79.0 & 76.0 & 86.2 & 98.9 & 95.5 & 85.1 & 86.8 & 77.7 & 71.9 & 69.9 & 977.1 & 4.840 & 1.010 \\
\hline Niles Twp. & 7.3 & 3.4 & 4.0 & 4.3 & 5.8 & 9.6 & 12.3 & 5.1 & 6.9 & 3.8 & 3.6 & 3.8 & 69.9 & .816 & .061 \\
\hline Watervliet & 6.7 & 5.5 & 5.9 & 7.0 & 7.0 & 7.4 & 8.8 & 8.7 & 9.3 & 7.6 & 5.5 & 5.9 & 85.3 & .884 & .039 \\
\hline \multicolumn{16}{|l|}{ BRANCH } \\
\hline $\begin{array}{l}\text { Bronson } \\
\text { Coldwater }\end{array}$ & $\begin{array}{r}29.1 \\
103.0\end{array}$ & $\begin{array}{l}28.2 \\
86.5\end{array}$ & $\begin{array}{l}34.9 \\
96.5\end{array}$ & $\begin{array}{l}28.7 \\
90.3\end{array}$ & $\begin{array}{l}35.1 \\
99.8\end{array}$ & $\begin{array}{r}35.3 \\
132.1\end{array}$ & $\begin{array}{r}31.8 \\
127.4\end{array}$ & $\begin{array}{r}30.7 \\
104.8\end{array}$ & $\begin{array}{r}29.2 \\
105.7\end{array}$ & $\begin{array}{l}29.9 \\
93.8\end{array}$ & $\begin{array}{l}26.4 \\
86.2\end{array}$ & $\begin{array}{l}27.7 \\
83.3\end{array}$ & $\begin{array}{r}367.0 \\
1,209.4\end{array}$ & $\begin{array}{l}1.455 \\
6.611\end{array}$ & $\begin{array}{r}.427 \\
1.976\end{array}$ \\
\hline Quincy & & & & & & & & & & & & & e) 72.2 & & \\
\hline $\begin{array}{l}\text { Reg. Center Dev. Disab. } \\
\text { Union City }\end{array}$ & $\begin{array}{l}7.2 \\
4.1\end{array}$ & $\begin{array}{l}6.5 \\
7.5\end{array}$ & $\begin{array}{l}8.0 \\
5.3\end{array}$ & $\begin{array}{l}7.5 \\
4.7\end{array}$ & $\begin{array}{l}7.2 \\
4.8\end{array}$ & $\begin{array}{l}6.9 \\
5.5\end{array}$ & $\begin{array}{l}7.1 \\
6.8\end{array}$ & $\begin{array}{l}6.5 \\
4.6\end{array}$ & $\begin{array}{l}5.9 \\
5.0\end{array}$ & $\begin{array}{l}5.9 \\
3.9\end{array}$ & $\begin{array}{l}5.6 \\
4.9\end{array}$ & $\begin{array}{l}6.2 \\
3.4\end{array}$ & $\begin{array}{l}80.5 \\
60.5\end{array}$ & $\begin{array}{l}.321 \\
.512\end{array}$ & $\begin{array}{l}.124 \\
.105\end{array}$ \\
\hline \multicolumn{16}{|l|}{ CALHOUN } \\
\hline Albion & 111.8 & 94.4 & 104.5 & 97.9 & 103.8 & $\begin{aligned} 115.5 \\
3.9\end{aligned}$ & 102.0 & 91.5 & 96.1 & 98.9 & 89.8 & 84.6 & $1,190.8$ & 4.839 & 1.386 \\
\hline 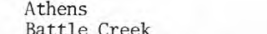 & & 2.4 & 2.7 & 2.4 & 2.9 & 3.9 & 4.6 & 3.0 & 3.0 & 2.8 & 2.6 & 3.3 & 36.2 & .154 & .060 \\
\hline Battle Creek & 283.4 & 220.0 & 203.6 & 187.6 & 245.9 & 265.7 & 293.7 & 257.0 & 261.3 & 227.4 & 212.9 & 216.4 & $2,874.9$ & 14.030 & 4.160 \\
\hline Battle Creek Twp. & 46.5 & 48.0 & 50.5 & 47.6 & 56.4 & 75.3 & 93.7 & 58.7 & 67.4 & 53.5 & 44.3 & 43.3 & 685.2 & 4.263 & 1.101 \\
\hline Homer & 6.3 & 6.9 & 7.2 & 4.7 & 9.2 & 11.6 & 6.6 & 5.6 & 5.0 & 5.2 & 4.1 & 4.4 & 76.8 & .496 & .091 \\
\hline Marshall & 33.2 & 34.0 & 37.9 & 34.0 & 35.8 & 42.7 & 44.3 & 39.5 & 39.3 & 34.9 & 30.8 & 30.0 & 436.4 & 1.825 & .748 \\
\hline \multicolumn{16}{|l|}{ CASS } \\
\hline Cassopolis & 5.6 & 5.1 & 5.3 & 5.5 & 5.8 & 5.8 & 5.8 & 5.5 & 6.1 & 5.3 & 5.4 & 5.3 & 66.5 & .264 & .126 \\
\hline Dowagiac & 28.9 & 27.0 & 29.6 & 27.2 & 30.7 & 33.8 & 39.7 & 34.3 & 28.8 & 29.2 & 24.2 & 23.5 & 356.9 & 2.164 & .316 \\
\hline \multicolumn{16}{|l|}{ CHARLEVOIX } \\
\hline $\begin{array}{l}\text { Boyne City } \\
\text { East Jordan }\end{array}$ & $\begin{array}{l}11.2 \\
16.1\end{array}$ & $\begin{array}{l}11.3 \\
14.6\end{array}$ & $\begin{array}{l}12.9 \\
17.6\end{array}$ & $\begin{array}{l}11.2 \\
18.8\end{array}$ & 12.2 & $\begin{array}{l}13.2 \\
18.6\end{array}$ & $\begin{array}{l}14.0 \\
22.2\end{array}$ & $\begin{array}{l}12.8 \\
20.2\end{array}$ & $\begin{array}{l}18.8 \\
18.6\end{array}$ & $\begin{array}{l}18.0 \\
18.3\end{array}$ & $\begin{array}{l}15.2 \\
18.7\end{array}$ & $\begin{array}{l}17.2 \\
16.1\end{array}$ & $\begin{array}{l}168.0 \\
220.9\end{array}$ & $\begin{array}{r}.824 \\
1.100\end{array}$ & $\begin{array}{l}.301 \\
.320\end{array}$ \\
\hline \multicolumn{16}{|l|}{ CHEBOYGAN } \\
\hline Mackinaw City & 2.9 & 5.1 & 5.6 & 4.2 & 5.5 & 8.0 & 11.9 & 11.4 & 7.3 & 5.3 & 2.7 & 2.8 & 72.7 & .525 & .076 \\
\hline $\begin{array}{l}\text { CHIPPEWA } \\
\text { Kincheloe AFB }\end{array}$ & 7.5 & 6.7 & 7.7 & 9.5 & 8.8 & 9.9 & 10.4 & 14.8 & 8.2 & 7.0 & 8.2 & 10.0 & 108.7 & .884 & .067 \\
\hline
\end{tabular}


TABLE 2. REPORTED GROUND-WATER PUMPAGE, IN 1979. (IN MILLIONS OF GALLONS)-continUed

\begin{tabular}{|c|c|c|c|c|c|c|c|c|c|c|c|c|c|c|c|}
\hline $\begin{array}{c}\text { COUNTY } \\
\text { AND } \\
\text { WATER USER }\end{array}$ & JAN & FEB & MAR & APR & MAY & $\pi \mathbb{N}$ & $\pi L$ & AUG & SEP & OCT & NOV & $\mathrm{DEC}$ & $\begin{array}{l}1979 \\
\text { TOTAL }\end{array}$ & $\begin{array}{l}\text { MAX } \\
\text { DAY }\end{array}$ & $\begin{array}{l}\text { MIN } \\
\text { DAY }\end{array}$ \\
\hline \multicolumn{16}{|l|}{ CLARE } \\
\hline & 26.8 & 20.6 & 23.1 & 21.1 & 24.4 & 36.3 & 59.6 & 30.0 & 39.6 & 30.3 & 19.7 & 20.5 & 352.0 & 2.392 & .580 \\
\hline Farwell & 5.9 & 4.4 & 4.5 & 4.2 & 5.9 & 9.3 & 9.9 & 7.5 & 6.2 & 6.1 & 4.9 & 6.0 & 74.8 & .463 & .074 \\
\hline Harrison & 6.6 & 10.8 & 10.8 & 6.1 & 8.7 & 11.1 & 15.4 & 9.2 & 6.2 & 6.9 & 5.7 & 5.1 & 102.6 & .641 & .166 \\
\hline \multicolumn{16}{|l|}{ CLINTON } \\
\hline Fowler & 2.1 & .2 .0 & 2.6 & 2.4 & 2.7 & 2.7 & 3.1 & 2.7 & 2.9 & 3.0 & 2.3 & 1.6 & 30.1 & -- & -. \\
\hline Maple Rapids & 1.7 & 1.8 & 2.1 & 1.9 & 1.8 & 2.1 & 2.3 & 2.5 & 3.1 & 1.8 & 1.7 & 1.7 & 24.5 & .103 & .053 \\
\hline Ovid & 5.6 & 5.7 & 5.9 & 6.0 & 6.6 & 7.0 & 7.0 & 6.3 & 6.5 & 6.3 & 6.3 & 6.7 & 75.9 & .296 & .123 \\
\hline St. Johns & 44.8 & 44.8 & 49.1 & 43.8 & 45.8 & 47.7 & 46.8 & 36.8 & 35.2 & 47.7 & 38.1 & 30.6 & 511.2 & 2.303 & .511 \\
\hline Westphalia & 1.7 & 1.6 & 1.7 & 1.9 & 1.8 & 1.9 & 1.9 & 1.9 & 1.9 & 1.7 & 1.7 & 1.7 & e) 21.4 & - & $\cdots$ \\
\hline \multicolumn{16}{|l|}{ CRAWFORD } \\
\hline Grayling & 13.4 & 13.1 & 15.2 & 14.4 & 16.6 & 17.3 & 20.0 & 18.0 & 17.8 & 17.6 & 17.0 & 18.5 & 198.9 & .942 & .304 \\
\hline \multicolumn{16}{|l|}{ DICKINSON } \\
\hline Breitung Twp. & 3.0 & 3.3 & 3.9 & 3.9 & 4.1 & 3.7 & 4.5 & 3.3 & 3.4 & 3.7 & 3.8 & 4.0 & 44.6 & .228 & .042 \\
\hline \multicolumn{16}{|l|}{ EATON } \\
\hline Bellevue & 4.0 & 4.3 & 4.9 & 3.9 & 5.6 & 4.6 & 6.5 & 3.7 & 5.3 & 4.9 & 3.7 & 3.6 & 55.0 & .285 & .070 \\
\hline Charlotte & 40.6 & 35.6 & 39.1 & 36.0 & 38.8 & 42.4 & 41.9 & 29.2 & 33.7 & 32.2 & 32.9 & 33.6 & 436.0 & 2.374 & .330 \\
\hline De1ta Twp. & 62.5 & 61.7 & 52.9 & 51.6 & 62.5 & 86.6 & 87.6 & 66.9 & 81.7 & 66.9 & 60.7 & 58.4 & 800.0 & -- & -- \\
\hline Eaton Rapids & 32.0 & 26.3 & 27.9 & 28.9 & 34.9 & 37.0 & 39.7 & 37.0 & 32.7 & 32.3 & 25.0 & 23.0 & 376.7 & 1.600 & .700 \\
\hline Grand Ledge & 17.9 & 19.0 & 18.5 & 17.2 & 18.9 & 20.5 & 24.2 & 18.0 & 20.8 & 17.9 & 16.7 & 14.7 & 224.3 & -. & -. \\
\hline Olds Warehouse & .4 & .4 & .4 & .4 & .4 & .4 & .3 & .2 & .3 & .4 & .4 & .3 & 4.3 & $-\cdot$ & -- \\
\hline \multicolumn{16}{|l|}{ EMMET } \\
\hline $\begin{array}{l}\text { Harbor Springs } \\
\text { Petosky }\end{array}$ & 11.1 & 10.7 & 11.7 & 12.0 & 14.5 & 17.1 & 34.6 & 21.5 & 17.9 & 13.3 & 11.4 & 12.1 & 187.9 & 1.837 & .322 \\
\hline Petosky & 40.3 & 33.9 & 42.2 & 43.1 & 47.2 & 47.5 & 58.2 & 50.4 & 43.1 & 43.3 & 39.8 & 41.1 & 530.1 & 2.354 & 1.026 \\
\hline \multicolumn{16}{|l|}{ GENESEE } \\
\hline $\begin{array}{l}\text { Beecher Metro. Dist. } \\
\text { Burton }\end{array}$ & 41.9 & 35.6 & 38.3 & $\begin{array}{l}35.5 \\
25.6\end{array}$ & 38.8 & 45.4 & 44.7 & 40.2 & 40.0 & 39.6 & 35.3 & 38.4 & 473.7 & 2.098 & .798 \\
\hline $\begin{array}{l}\text { Burton } \\
\text { Davison }\end{array}$ & $\begin{array}{l}23.5 \\
20.0\end{array}$ & 21.3 & 26.8 & 25.6 & 29.8 & 31.5 & 29.1 & 30.5 & 31.2 & $\begin{array}{l}33.2 \\
26.1\end{array}$ & $\begin{array}{l}29.2 \\
22.2\end{array}$ & 30.1 & 341.8 & 1.622 & .099 \\
\hline $\begin{array}{l}\text { Davison } \\
\text { Fenton }\end{array}$ & 28.6 & $\begin{array}{l}18.7 \\
24.9\end{array}$ & $\begin{array}{l}22.7 \\
26.9\end{array}$ & $\begin{array}{l}20.8 \\
25.1\end{array}$ & $\begin{array}{l}22.5 \\
27.6\end{array}$ & $\begin{array}{l}24.8 \\
31.7\end{array}$ & $\begin{array}{l}27.3 \\
32.1\end{array}$ & $\begin{array}{l}22.5 \\
30.0\end{array}$ & $\begin{array}{l}22.9 \\
28.6\end{array}$ & $\begin{array}{l}26.1 \\
24.4\end{array}$ & 29.1 & $\begin{array}{l}24.7 \\
25.8\end{array}$ & $\begin{array}{l}275.0 \\
334.8\end{array}$ & 1.985 & .499 \\
\hline Grand Blanc & 35.5 & 28.4 & 30.8 & 29.5 & 33.6 & 48.9 & 46.2 & 35.6 & 40.6 & 33.3 & 28.6 & 32.8 & 423.8 & 2.987 & .766 \\
\hline Linden & 4.0 & 4.2 & 4.2 & 4.2 & 4.9 & 5.3 & 5.8 & 6.0 & 5.6 & 4.7 & 5.1 & 5.1 & 59.1 & .272 & .074 \\
\hline Montrose & 5.5 & 6.2 & 6.5 & 5.7 & 5.9 & 6.4 & 6.1 & 5.9 & 6.3 & 6.4 & 6.0 & 6.1 & 73.0 & .364 & .126 \\
\hline \multicolumn{16}{|l|}{ GLADWIN } \\
\hline Beaverton & 3.7 & 3.3 & 4.0 & 3.3 & 4.0 & 4.0 & 4.9 & 4.2 & 3.6 & 4.2 & 3.4 & 3.3 & 45.9 & $-\cdot$ & -- \\
\hline \multicolumn{16}{|l|}{ GOGEBIC } \\
\hline Ironwood & 45.4 & 46.9 & 53.3 & 51.5 & 45.1 & 39.9 & 42.6 & 44.3 & 40.4 & 39.5 & 38.0 & 42.3 & 529.2 & $\because-$ & 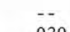 \\
\hline Marenisco Twp. & 6.9 & 7.1 & 7.8 & 5.9 & 6.3 & 5.9 & 6.6 & 6.0 & 5.2 & 5.6 & 6.1 & 7.0 & 76.4 & .592 & .020 \\
\hline Wakefield & 8.9 & 9.3 & 10.9 & 8.7 & 8.6 & 7.7 & 9.3 & 8.1 & 8.0 & 8.4 & 8.4 & 9.2 & 105.5 & .869 & .207 \\
\hline \multicolumn{16}{|l|}{ GRAND TRAVERSE } \\
\hline Kingsley & 1.9 & 1.7 & 2.1 & 2.9 & 2.8 & 4.3 & 4.6 & 3.7 & 3.1 & 2.8 & 3.4 & 2.3 & e) 35.6 & $-\cdot$ & -- \\
\hline \multicolumn{16}{|l|}{ GRATIOT } \\
\hline $\begin{array}{l}\text { Breckenridge } \\
\text { Ithaca }\end{array}$ & $\begin{array}{l}2.9 \\
8.6\end{array}$ & 2.7 & 3.1 & 2.9 & $\begin{array}{r}3.2 \\
10.7\end{array}$ & 3.3 & 3.6 & $\begin{array}{r}3.0 \\
10.9\end{array}$ & $\begin{array}{l}3.0 \\
8.9\end{array}$ & 3.1 & 2.8 & 2.8 & 36.4 & $\begin{array}{r}.159 \\
437\end{array}$ & .046 \\
\hline $\begin{array}{l}\text { Ithaca } \\
\text { St. Louis }\end{array}$ & $\begin{array}{r}0.0 \\
12.7\end{array}$ & 11.8 & $\begin{array}{r}3.5 \\
13.0\end{array}$ & $\begin{array}{r}9.8 \\
13.9\end{array}$ & $\begin{array}{l}10.7 \\
14.1\end{array}$ & $\begin{array}{l}11.0 \\
16.3\end{array}$ & $\begin{array}{r}9.7 \\
18.5\end{array}$ & $\begin{array}{l}10.9 \\
13.8\end{array}$ & $\begin{array}{r}8.9 \\
13.1\end{array}$ & $\begin{array}{l}10.0 \\
14.9\end{array}$ & $\begin{array}{r}9.4 \\
11.8\end{array}$ & $\begin{array}{r}9.4 \\
11.8\end{array}$ & 165.7 & .789 & .279 \\
\hline \multicolumn{16}{|l|}{ HILLS } \\
\hline Camden & 1.6 & 1.4 & 1.3 & 1.2 & 1.6 & 1.6 & 1.6 & 1.6 & 1.4 & 1.2 & 1.2 & 1.1 & 16.8 & .070 & .022 \\
\hline Hillsdale & 39.7 & 36.4 & 40.9 & 39.8 & 44.3 & 45.3 & 44.8 & 42.1 & 44.2 & 42.1 & 40.3 & 38.7 & 498.6 & 1.975 & .948 \\
\hline Jonesville & 18.4 & 13.2 & 16.0 & 14.1 & 14.0 & 14.4 & 13.9 & 9.9 & 10.5 & 11.6 & 11.3 & 12.4 & 159.7 & .988 & .127 \\
\hline Litchfield & 3.6 & 3.1 & 3.1 & 3.7 & 4.5 & 5.4 & 4.5 & 4.4 & 3.2 & 2.8 & 3.3 & 2.8 & 44.4 & .222 & .074 \\
\hline Waldron & 2.3 & 2.3 & 2.3 & 2.5 & 2.6 & 2.6 & 2.9 & 2.9 & 3.2 & 3.0 & 2.7 & 2.9 & 32.2 & .169 & .015 \\
\hline \multicolumn{16}{|l|}{ HOUGHTON } \\
\hline $\begin{array}{l}\text { a)Adams Twp. - S. Range } \\
\text { Water Auth. }\end{array}$ & & & & & & & & & & & & & & & \\
\hline $\begin{array}{l}\text { Water Auth. } \\
\text { b)Adams Twp. - s. Range }\end{array}$ & 47.0 & 29.5 & 28.7 & 28.2 & 26.4 & 25.4 & 35.2 & 26.7 & 28.3 & 29.3 & 27.6 & 26.9 & e) 359.2 & 2.194 & .546 \\
\hline $\begin{array}{l}\text { Water Auth. } \\
\text { Chassell Twp. }\end{array}$ & 10.3 & 9.6 & 11.2 & 10.4 & 10.1 & 8.2 & 8.6 & 8.7 & 9.3 & 8.9 & 9.0 & 10.4 & e) 114.7 & .492 & .232 \\
\hline $\begin{array}{l}\text { Chasse11 Twp. } \\
\text { Houghton }\end{array}$ & $\begin{array}{r}3.1 \\
36.2\end{array}$ & $\begin{array}{r}3.4 \\
30.8\end{array}$ & $\begin{array}{r}3.9 \\
39.4\end{array}$ & $\begin{array}{r}3.1 \\
41.0\end{array}$ & $\begin{array}{r}2.9 \\
39.3\end{array}$ & $\begin{array}{r}2.8 \\
37.6\end{array}$ & $\begin{array}{r}3.4 \\
47.7\end{array}$ & $\begin{array}{r}2.9 \\
43.1\end{array}$ & $\begin{array}{r}2.7 \\
45.0\end{array}$ & $\begin{array}{r}3.1 \\
45.4\end{array}$ & $\begin{array}{r}2.2 \\
39.8\end{array}$ & $\begin{array}{r}2.5 \\
41.9\end{array}$ & $\begin{array}{r}36.0 \\
487.2\end{array}$ & .153 & .050 \\
\hline c) N. Michigan wate & 38.0 & 35.3 & 38.9 & 37.6 & 37.0 & 35.2 & 41.5 & 38.2 & 32.4 & 37.7 & 34.2 & 35.2 & 441.2 & 1.307 & 1.046 \\
\hline
\end{tabular}


TABLE 2. REPORTED GROUND-WATER PUMPAGE, IN 1979. (IN MILLIONS OF GALLONS)-continUed

\begin{tabular}{|c|c|c|c|c|c|c|c|c|c|c|c|c|c|c|c|}
\hline $\begin{array}{c}\text { COUNTY } \\
\text { AND } \\
\text { WATER USER }\end{array}$ & JAN & FEB & MAR & APR & MAY & JUN & JUL & AUG & SEP & OCT & NOV & $\mathrm{DEC}$ & $\begin{array}{l}1979 \\
\text { TOTAL }\end{array}$ & $\begin{array}{l}\text { MAX } \\
\text { DAY }\end{array}$ & $\begin{array}{l}\text { MIN } \\
\text { DAY }\end{array}$ \\
\hline \multicolumn{16}{|l|}{ HURON } \\
\hline Elkton & 1.4 & 1.2 & .8 & 2.3 & 1.3 & 2.0 & .8 & 1.6 & .7 & 1.7 & 1.7 & 1.7 & 17.2 & -- & -- \\
\hline Q) Pigeon. & & & 7.0 & & & 8.6 & & & 10.8 & & & 8.6 & 35.0 & -. & -- \\
\hline Sebewaing & 11.0 & 12.3 & 12.4 & 11.9 & 12.6 & 11.0 & 11.0 & 11.0 & 11.0 & 11.0 & 10.4 & 14.4 & e) 140.0 & -- & $-\cdot$ \\
\hline \multirow{2}{*}{\multicolumn{16}{|c|}{ INGHAM }} \\
\hline & & & & & & & 181.8 & 147.2 & 177.9 & 153.8 & 133.6 & 128.4 & $1,775.2$ & 9.651 & 3.582 \\
\hline $\begin{array}{l}\text { Lansing } \\
\text { Lant Mon }\end{array}$ & 775.0 & 742.7 & 803.2 & 759.0 & 849.4 & 950.6 & 900.6 & 797.4 & 828.9 & 796.8 & 721.9 & 648.6 & $9,574.1$ & 36.960 & 16.500 \\
\hline Lansing Twp. & 32.4 & 36.7 & 30.1 & 37.4 & 41.7 & 44.6 & 56.6 & 54.3 & 54.9 & 51.3 & 47.6 & 44.1 & 531.7 & -- & - \\
\hline Leslie & 7.4 & 7.0 & 8.6 & 8.2 & 9.3 & 13.0 & 12.0 & 9.6 & 8.8 & 8.0 & 6.8 & 5.9 & 104.6 & -- & -- \\
\hline Mason & 18.0 & 15.8 & 19.6 & 16.9 & 19.8 & 21.8 & 22.6 & 20.6 & 20.8 & 19.0 & 16.9 & 18.0 & 229.8 & 1.291 & .001 \\
\hline Michigan State Univ. & 155.8 & 134.1 & 138.5 & 151.3 & 163.5 & 146.9 & 136.9 & 127.4 & 142.7 & 154.2 & 133.5 & 93.9 & $1,678.7$ & 6.370 & 1.655 \\
\hline Oldsmobile Forge No. 2 & 9.8 & 9.4 & 8.7 & 7.5 & 7.1 & 7.8 & 4.7 & 4.5 & 4.9 & 8.3 & 7.0 & 5.5 & 85.2 & -- & -- \\
\hline Stockbridge & 4.7 & 5.7 & 4.9 & 4.3 & 5.1 & 5.5 & 1.1 & 4.0 & 3.8 & 3.6 & 3.4 & 3.8 & 49.9 & .226 & .060 \\
\hline Webberville & 3.8 & 3.7 & 3.7 & 3.8 & 4.5 & 5.9 & 6.5 & 3.8 & 4.1 & 3.7 & 3.3 & 3.6 & 50.4 & .354 & .084 \\
\hline Williamston & 7.3 & 7.4 & 7.8 & 7.0 & 8.0 & 9.7 & 10.4 & 8.1 & 8.2 & 7.3 & 6.5 & 7.2 & 94.9 & .548 & .150 \\
\hline \multicolumn{16}{|l|}{ IONIA, } \\
\hline Belding & 53.7 & 54.2 & 58.0 & 55.7 & 65.2 & 68.5 & 67.1 & 62.9 & 58.8 & 61.5 & 51.0 & 36.5 & 693.1 & & -- \\
\hline Ionia & 20.0 & 26.2 & 23.5 & 27.1 & 31.9 & 37.4 & 33.7 & 35.3 & 30.5 & 32.5 & 30.7 & 27.8 & 356.6 & 1.453 & .480 \\
\hline Mich. Reformatory, Ionia & 12.6 & 12.6 & 12.9 & 12.4 & 15.1 & 17.1 & 17.8 & 17.2 & 16.0 & 13.4 & 16.1 & 12.8 & 176.0 & -. & . \\
\hline Mich. Training Unit, Ionia & 5.1 & 4.9 & 4.8 & 6.2 & 7.2 & 8.4 & 5.3 & 7.9 & 9.7 & 6.5 & 6.0 & 6.3 & 78.3 & 1.351 & .035 \\
\hline Muir & 4.2 & 2.2 & 2.9 & 3.5 & 4.7 & 6.1 & 6.9 & 5.0 & 4.1 & 4.2 & 3.0 & 3.7 & 50.5 & .252 & .085 \\
\hline Pewamo & 1.1 & 1.2 & 1.5 & 1.2 & 1.3 & 2.5 & 2.4 & 1.6 & 1.8 & 1.3 & 1.3 & 1.5 & 18.7 & - & -. \\
\hline Portland & 15.2 & 10.7 & 12.3 & 13.8 & 14.4 & 15.6 & 15.1 & 12.8 & 15.5 & 12.3 & 11.6 & 11.6 & 160.9 & -- & -- \\
\hline Riverside Center & 3.7 & 6.2 & 7.5 & 6.8 & 7.5 & 7.7 & 7.9 & 7.0 & 5.8 & 6.2 & 6.3 & 6.9 & 79.5 & .444 & .115 \\
\hline Saranac & 13.2 & 11.7 & 13.3 & 11.9 & 11.8 & 12.1 & 12.3 & 11.6 & 9.8 & 8.7 & 8.4 & 7.8 & 132.6 & .606 & .107 \\
\hline \multicolumn{16}{|l|}{ IOSCO } \\
\hline Oscoda Twp. & 28.2 & 19.2 & 24.6 & 21.6 & 17.3 & 26.7 & 33.8 & 25.0 & 23.3 & 18.8 & 19.9 & 43.0 & 301.4 & $\cdots$ & -- \\
\hline Wurtsmith AFB & 18.9 & 16.2 & 19.7 & 21.3 & 23.0 & 24.4 & 32.2 & 25.8 & 19.2 & 16.9 & 15.8 & 16.7 & 250.1 & 1.538 & .286 \\
\hline \multicolumn{16}{|l|}{ IRON } \\
\hline Alpha & .9 & .8 & .8 & .9 & .9 & .8 & .9 & .8 & .9 & .8 & .8 & .9 & e) 10.2 & -- & -- \\
\hline Caspian & 9.4 & 8.9 & 9.8 & 9.3 & 10.0 & 9.1 & 11.4 & 10.0 & 9.3 & 8.6 & 7.6 & 8.4 & 111.8 & .475 & .194 \\
\hline Crystal Fa11s & 14.6 & 14.0 & 16.5 & 14.7 & 15.3 & 15.1 & 19.1 & 15.4 & 14.3 & 13.4 & 13.1 & 14.0 & 179.5 & .799 & .384 \\
\hline Crystal Falls Twp. & 8.6 & 8.2 & 7.8 & 6.7 & 6.4 & 6.5 & 9.1 & 7.8 & 6.3 & 7.2 & 6.0 & 9.3 & 89.9 & .329 & .201 \\
\hline Iron River & 13.6 & 16.3 & 18.9 & 15.6 & 12.4 & 10.3 & 11.7 & 11.1 & 9.6 & 9.1 & 8.9 & 9.5 & 147.0 & .862 & .180 \\
\hline Iron River Twp. & 10.9 & 10.1 & 11.6 & 10.7 & 9.0 & 9.9 & 13.6 & 10.7 & 9.2 & 10.1 & 9.7 & 10.8 & 126.3 & -- & -- \\
\hline Stambaugh & 6.7 & 6.2 & 7.7 & 6.2 & 5.8 & 5.6 & 6.3 & 6.0 & 5.8 & 5.6 & 5.1 & 5.3 & 72.3 & .529 & .131 \\
\hline Stambaugh Twp. & 7.0 & 5.4 & 7.0 & 5.4 & 4.0 & 3.4 & 4.8 & 4.6 & 3.1 & 3.5 & 3.0 & 4.0 & 55.2 & - & -- \\
\hline \multicolumn{16}{|l|}{ ISABELLA } \\
\hline d)Mt. Pleasant & 66.6 & 59.6 & 66.3 & 64.2 & 59.2 & 63.6 & 66.0 & 65.3 & 78.0 & 71.2 & 67.2 & 60.6 & 787.8 & 3.732 & 1.268 \\
\hline Shepherd & 3.9 & 5.5 & 5.9 & 4.2 & 4.6 & 4.1 & 4.6 & 4.1 & 3.9 & 4.3 & 3.7 & 3.6 & 52.4 & .218 & .005 \\
\hline \multicolumn{16}{|l|}{ JACKSON } \\
\hline $\begin{array}{l}\text { Concord } \\
\text { Grass Lake }\end{array}$ & $\begin{array}{l}3.6 \\
1.9\end{array}$ & $\begin{array}{l}3.0 \\
1.5\end{array}$ & 3.7 & $\begin{array}{l}3.3 \\
1.5\end{array}$ & $\begin{array}{l}4.1 \\
1.8\end{array}$ & 5.2 & 5.1 & $\begin{array}{l}3.5 \\
1.8\end{array}$ & 3.9 & 3.2 & 3.2 & 4.0 & 45.8 & .597 & .051 \\
\hline Jackson & $\begin{array}{r}1.9 \\
344.1\end{array}$ & $\begin{array}{l}1.5 \\
317.6\end{array}$ & 357.8 & $\begin{array}{l}1.5 \\
317.7\end{array}$ & $\begin{array}{r}1.8 \\
353.2\end{array}$ & 350.6 & $\begin{array}{r}2.6 \\
353.7\end{array}$ & $\begin{array}{r}1.8 \\
358.1\end{array}$ & 338.4 & $\begin{array}{r}1.9 \\
337.4\end{array}$ & $\begin{array}{r}1.8 \\
296.4\end{array}$ & 268.1 & $3,993.1$ & 16.180 & 5.460 \\
\hline Springport & $\begin{array}{r}r 44.1 \\
2.9\end{array}$ & 3.0 & 1.9 & 2.8 & 1.6 & 4.2 & .6 & 3.4 & .2 & .4 & 2.7 & $\begin{array}{r}200.1 \\
1.4\end{array}$ & 25.1 & & \\
\hline State Prison, Jackson & 48.2 & 42.0 & 46.5 & 44.9 & 50.0 & 49.5 & 53.7 & 54.7 & 46.8 & 54.1 & 45.3 & 51.7 & 587.4 & 2.370 & 1.300 \\
\hline \multicolumn{16}{|l|}{ KALAM } \\
\hline & 3.3 & 3.1 & 3.4 & 4.0 & 4.1 & 3.9 & 4.6 & 2.5 & 1.9 & 2.7 & 7.1 & 2.5 & 43.1 & .466 & .048 \\
\hline Brown & 263.9 & 221.7 & 245.0 & 237.5 & 246.7 & 251.2 & 228.7 & 242.4 & 243.1 & 270.0 & 228.2 & 237.0 & $2,915.4$ & -- & -- \\
\hline $\mathrm{Ga}$ & 6.0 & 5.7 & 5.7 & 6.4 & 7.0 & 7.7 & 8.6 & 7.0 & 9.0 & 7.9 & 5.4 & 6.2 & 82.6 & .380 & .154 \\
\hline Kala & 467.7 & 421.8 & 463.9 & 473.5 & 550.5 & 670.5 & 780.6 & 576.6 & 647.0 & 535.7 & 471.3 & 460.7 & $6,519.8$ & 36.043 & 10.453 \\
\hline Parcl & 6.5 & 6.9 & 6.5 & 6.8 & 8.6 & 10.6 & 14.5 & 8.0 & 10.3 & 6.3 & 5.6 & 6.1 & 96.7 & 1.034 & .096 \\
\hline Portag & 52.9 & 50.7 & 59.5 & 56.8 & 78.1 & 112.1 & 137.7 & 67.7 & 96.8 & 65.8 & 58.5 & 56.4 & 893.0 & 7.609 & .792 \\
\hline School & 5.6 & 6.6 & 7.0 & 6.1 & 8.6 & 8.9 & 8.9 & 6.3 & 6.1 & 5.9 & 5.6 & 7.0 & e) 82.6 & - & -- \\
\hline Simpson-Lee Paper Co. & 27.9 & 25.6 & 26.6 & 26.4 & 25.8 & 26.7 & 20.5 & 26.6 & 22.6 & 27.0 & 23.7 & 26.9 & 306.3 & -- & $-\cdot$ \\
\hline f) State Hosp., Kalamazoo & 10.6 & 7.3 & 9.5 & 7.5 & 8.0 & 2.7 & & & & & & & 45.6 & .459 & .189 \\
\hline Upjohn Co. & 520.7 & 473.6 & 575.5 & 535.4 & 605.8 & 583.3 & 596.1 & 553.2 & 515.5 & 526.7 & 490.0 & 513.9 & $6,489.7$ & 24.179 & 12.249 \\
\hline Vicksburg & 9.5 & 8.9 & 9.8 & 9.4 & 11.4 & 13.8 & 16.8 & 16.9 & 12.3 & 9.3 & 8.6 & 9.5 & 136.2 & .880 & .140 \\
\hline KALKASKA & & & & & & & & & & & & & & & \\
\hline Kalkaska & 16.1 & 17.4 & 17.6 & 15.3 & 16.2 & 17.7 & 20.4 & 18.1 & 15.7 & 13.0 & 11.3 & 13.7 & 192.5 & .755 & .283 \\
\hline KENT & & & & & & & & & & & & & & & \\
\hline Alloytek Inc. & 9.6 & 7.9 & 8.8 & 8.2 & 9.0 & 10.4 & 11.8 & 11.6 & 10.2 & 8.7 & 7.3 & 7.7 & 111.2 & .480 & .117 \\
\hline Cedar Springs & 12.1 & 11.0 & 10.8 & 9.9 & 10.4 & 11.4 & 14.3 & 10.5 & 11.0 & 10.3 & 9.4 & 9.3 & 130.4 & $-\cdot$ & -- \\
\hline $\begin{array}{l}\text { Kent County Airp } \\
\text { Lowe11 }\end{array}$ & 19.0 & 18.1 & 19 & 18.8 & 21. & 25.2 & 25 & 22. & 21.4 & 20.9 & 18.1 & 16.8 & 246.3 & 1.276 & .172 \\
\hline Plainfield Twp. & 37.5 & 33.5 & 37.0 & 36.3 & 56.2 & 85.9 & 92. & 54.5 & 68.6 & 41.2 & 37.7 & 38.0 & 619.0 & 4.894 & .770 \\
\hline Sparta & 8.9 & 8.2 & 9.0 & 9.7 & 11.1 & 12.7 & 14.5 & 11.4 & 12.2 & 10.9 & 9.3 & 9.7 & 127.6 & .648 & .140 \\
\hline
\end{tabular}


TABLE 2. REPORTED GROUND-WATER PUMPAGE, IN 1979. (IN MILLIONS OF GALLONS)-Continued

\begin{tabular}{|c|c|c|c|c|c|c|c|c|c|c|c|c|c|c|c|}
\hline $\begin{array}{c}\text { COUNTY } \\
\text { AND } \\
\text { WATER USER }\end{array}$ & JAN & FEB & MAR & APR & MAY & JUN & JUL & AUG & SEP & OCT & NOV & $\mathrm{DEC}$ & $\begin{array}{l}1979 \\
\text { TOTAL }\end{array}$ & $\begin{array}{l}\text { MAX } \\
\text { DAY }\end{array}$ & $\begin{array}{l}\text { MIN } \\
\text { DAY }\end{array}$ \\
\hline \multicolumn{16}{|l|}{ LAKE } \\
\hline Baldwin & 2.3 & 3.2 & 4.3 & 2.1 & 4.9 & 5.6 & 6.8 & 6.4 & 4.1 & 3.0 & 3.0 & 4.9 & 50.6 & .306 & -- \\
\hline \multicolumn{16}{|l|}{ LAPEER } \\
\hline Columbiaville & 4.3 & 3.9 & 4.3 & 4.3 & 4.4 & 4.6 & 4.5 & 4.3 & 4.4 & 3.8 & 4.0 & 2.5 & 50.3 & -- & -- \\
\hline Q) Dryden & & & 3.6 & & & 3.6 & & & 3.4 & & & 3.6 & e) 14.2 & -- & -- \\
\hline North Branch & 3.3 & 3.8 & 3.2 & 2.8 & 2.4 & 3.6 & 3.5 & 3.3 & 2.6 & 3.2 & 2.6 & 3.4 & 37.7 & .166 & .079 \\
\hline \multicolumn{16}{|l|}{ LEELANAU } \\
\hline Northport & 2.0 & 2.4 & 3.1 & 2.2 & 2.8 & 3.6 & 6.5 & 4.3 & 4.8 & 2.8 & 2.5 & 2.2 & 39.2 & .329 & .040 \\
\hline \multicolumn{16}{|l|}{ LENAWEE } \\
\hline Addison & 2.6 & 2.9 & 3.3 & 2.8 & 3.4 & 3.7 & 3.2 & 3.2 & 3.4 & 3.1 & 3.0 & 2.7 & 37.3 & -- & -- \\
\hline Britton & 1.4 & 1.3 & 1.4 & 1.3 & 1.3 & 1.6 & 1.3 & 1.4 & 1.3 & 1.5 & 1.4 & 1.8 & 17.0 & -. & .032 \\
\hline Clinton & 7.2 & 9.6 & 10.2 & 12.8 & 10.4 & 13.0 & 12.2 & 10.3 & 9.3 & 8.0 & 7.9 & 8.1 & 119.0 & -- & -- \\
\hline Hudson & 9.8 & 8.6 & 9.9 & 10.3 & 11.1 & 12.1 & 11.2 & 10.8 & 10.5 & 9.5 & 8.6 & 8.5 & 120.9 & .666 & .185 \\
\hline Morenci & 8.1 & 7.6 & 9.1 & 9.4 & 9.8 & 9.8 & 8.7 & 8.1 & 6.9 & 7.4 & 6.8 & 7.1 & 98.8 & .505 & .160 \\
\hline Onsted & 3.2 & 3.6 & 3.1 & 2.9 & 3.5 & 3.8 & 3.4 & 2.8 & 3.4 & 3.6 & 3.3 & 3.3 & 39.9 & -- & -- \\
\hline Tecumseh & 29.2 & 17.2 & 20.0 & 21.5 & 24.3 & 22.9 & 24.0 & 26.8 & 23.6 & 26.2 & 28.3 & 31.1 & 295.1 & 1.407 & .095 \\
\hline Fisher Body, Tecumseh & .8 & .8 & 1.0 & .9 & 1.1 & 1.0 & .7 & .9 & .8 & .9 & .8 & .5 & 10.2 & .214 & .000 \\
\hline \multicolumn{16}{|l|}{ LIVINGSTON } \\
\hline Brighton & 24.4 & 21.0 & 24.5 & 23.3 & 27.3 & 33.7 & 29.6 & 25.0 & 26.4 & 25.0 & 22.3 & 26.3 & 308.8 & 1.600 & .049 \\
\hline Fowlerville & 17.8 & 14.9 & 16.3 & 16.5 & 17.1 & 15.8 & 16.4 & 27.3 & 22.5 & 24.6 & 20.7 & 22.7 & 232.6 & 1.116 & .384 \\
\hline Green Oak Twp. & 2.0 & 2.1 & 2.1 & 1.7 & 2.6 & 4.7 & 3.6 & 2.6 & 3.5 & 2.2 & 2.1 & 2.4 & 31.6 & .270 & .028 \\
\hline Hillcrest Center, Howell & 3.0 & 2.7 & 2.8 & 2.3 & 2.5 & 3.1 & 3.2 & 3.2 & 3.4 & 3.2 & 2.8 & 3.1 & 35.3 & .179 & .032 \\
\hline Howell & 34.8 & 31.0 & 35.6 & 33.2 & 41.1 & 43.4 & 45.3 & 37.8 & 35.2 & 34.3 & 33.1 & 33.8 & e) 438.6 & 1.911 & .850 \\
\hline Maxey Boys Schoo1 & 3.8 & 3.7 & 3.7 & 3.5 & 3.8 & 3.9 & 3.7 & 3.7 & 3.4 & 3.7 & 3.7 & 3.6 & 44.2 & .193 & .044 \\
\hline \multicolumn{16}{|l|}{ LUCE } \\
\hline Newberry Health Center & 4.1 & 3.7 & 4.6 & 4.9 & 5.6 & 4.2 & 4.0 & 3.7 & 3.5 & 3.9 & 3.8 & . 4.6 & 50.6 & - & - \\
\hline \multicolumn{16}{|l|}{ MACOMB } \\
\hline Richmond & 11.0 & 11.6 & 11.5 & 13.1 & 11.8 & 13.2 & 11.8 & 12.8 & 11.8 & 12.3 & 10.8 & 10.8 & 142.5 & - & - \\
\hline \multicolumn{16}{|l|}{ MANISTEE } \\
\hline Filer Twp. & 3.4 & 2.9 & 3.7 & 3.6 & 4.5 & 7.6 & 9.7 & 5.1 & 4.8 & 3.5 & 3.7 & 3.9 & 56.4 & .525 & .000 \\
\hline Manistee & 42.4 & 41.7 & 46.4 & 41.9 & 45.8 & 56.4 & 68.1 & 48.8 & 50.5 & 45.6 & 43.6 & 45.2 & 576.4 & 3.149 & 1.092 \\
\hline \multicolumn{16}{|l|}{ MARQUETTE } \\
\hline Ishpeming Twp. & 9.9 & 9.5 & 11.6 & 11.1 & 11.2 & 10.0 & 13.8 & 10.5 & 9.2 & 9.1 & 9.1 & 9.7 & 124.7 & -- & -- \\
\hline K. I. Sawyer AFB & 37.7 & 35.3 & 38.3 & 35.7 & 37.7 & 33.1 & 49.1 & 39.5 & 35.1 & 35.6 & 34.8 & 36.9 & 448.8 & 2.095 & .958 \\
\hline \multicolumn{16}{|l|}{ MENOMINEE } \\
\hline Stephenson & 2.7 & 2.7 & 3.3 & 3.1 & 3.3 & 3.2 & 5.1 & 3.2 & 3.0 & 3.2 & 2.9 & 2.9 & 38.6 & .264 & .070 \\
\hline \multicolumn{16}{|l|}{ MIDLAND } \\
\hline Coleman & 4.4 & 5.0 & 4.8 & 3.6 & 4.6 & 4.2 & 5.8 & 4.0 & 3.7 & 3.5 & 3.2 & 3.7 & 50.5 & .270 & .080 \\
\hline \multicolumn{16}{|l|}{ MISSAUKEE } \\
\hline Lake City & 5.1 & 4.7 & 5.8 & 3.4 & 4.1 & 7.2 & 9.0 & 5.0 & 5.5 & 4.6 & 3.8 & 4.2 & 62.4 & .911 & .086 \\
\hline \multicolumn{16}{|l|}{ MONROE } \\
\hline Milan & 15.8 & 20.6 & 19.6 & 18.4 & 18.6 & 22.0 & 23.6 & 20.0 & 18.6 & 18.5 & 17.8 & 17.0 & 230.5 & -- & -- \\
\hline Petersburg & 2.9 & 2.9 & 3.1 & 2.9 & 2.8 & 3.0 & 3.3 & 3.5 & 2.8 & 2.8 & 2.5 & 2.7 & 35.2 & - & -- \\
\hline \multicolumn{16}{|l|}{ MONTCALM } \\
\hline Carson City & $\begin{array}{l}5.9 \\
3.8\end{array}$ & $\begin{array}{l}5.4 \\
3.8\end{array}$ & 5.8 & 5.8 & 6.5 & 6.2 & 6.5 & 6.4 & 6.4 & 6.0 & 6.8 & 5.3 & 73.0 & .330 & .140 \\
\hline $\begin{array}{l}\text { Edmore } \\
\text { Greenville }\end{array}$ & $\begin{array}{r}5.8 \\
73.1\end{array}$ & $\begin{array}{r}3.8 \\
67.1\end{array}$ & $\begin{array}{r}4.2 \\
73.9\end{array}$ & $\begin{array}{r}4.6 \\
71.1\end{array}$ & $\begin{array}{r}5.8 \\
83.6\end{array}$ & $\begin{array}{r}8.7 \\
90.2\end{array}$ & $\begin{array}{r}9.8 \\
90.0\end{array}$ & $\begin{array}{r}6.0 \\
77.2\end{array}$ & $\begin{array}{r}7.2 \\
84.5\end{array}$ & $\begin{array}{r}3.8 \\
83.9\end{array}$ & $\begin{array}{r}3.6 \\
63.6\end{array}$ & $\begin{array}{r}3.7 \\
63.3\end{array}$ & $\begin{array}{r}05.0 \\
921.5\end{array}$ & 4.006 & 1.258 \\
\hline Howard City & 2.2 & 2.1 & 2.5 & 2.1 & 1.9 & 2.7 & 2.9 & 2.0 & 1.6 & 1.8 & 1.8 & 2.6 & 26.2 & .128 & .038 \\
\hline Sheridan & 1.7 & 1.9 & 2.4 & 1.7 & 2.6 & 4.0 & 5.7 & 2.9 & 3.0 & 1.8 & 1.8 & 1.9 & 31.4 & -. & -. \\
\hline Stanton & 7.7 & 5.6 & 7.1 & 7.1 & 5.5 & 5.8 & 5.6 & 4.6 & 4.2 & 4.5 & 5.0 & 5.2 & 67.9 & $\cdots$ & -- \\
\hline
\end{tabular}


TABLE 2. REPORTED GROUND-WATER PUMPAGE, IN 1979. (IN MILLIONS OF GALLONS)-CONTINUED

\begin{tabular}{|c|c|c|c|c|c|c|c|c|c|c|c|c|c|c|c|}
\hline $\begin{array}{c}\text { COUNTY } \\
\text { AND } \\
\text { WATER USER }\end{array}$ & JAN & FEB & MAR & APR & MAY & JUN & JUL & AUG & SEP & OCT & NoV & $\mathrm{DEC}$ & $\begin{array}{l}1979 \\
\text { TOTAL }\end{array}$ & $\begin{array}{l}\text { MAX } \\
\text { DAY }\end{array}$ & $\begin{array}{l}\text { MIN } \\
\text { DAY }\end{array}$ \\
\hline \multicolumn{16}{|l|}{ MUSKEGON } \\
\hline Montague & 6.0 & 7.2 & 7.0 & 5.6 & 7.2 & 13.1 & 16.8 & 7.4 & 8.0 & 5.9 & 5.2 & 6.1 & 95.5 & -- & -- \\
\hline $\begin{array}{l}\text { Ravenna } \\
\text { Whiteha11 }\end{array}$ & $\begin{array}{r}2.3 \\
37.8\end{array}$ & $\begin{array}{r}2.1 \\
36.6\end{array}$ & $\begin{array}{r}2.3 \\
44.9\end{array}$ & $\begin{array}{r}2.3 \\
38.6\end{array}$ & $\begin{array}{r}2.5 \\
45.5\end{array}$ & $\begin{array}{r}2.9 \\
52.8\end{array}$ & $\begin{array}{r}3.9 \\
60.0\end{array}$ & $\begin{array}{r}2.8 \\
45.6\end{array}$ & $\begin{array}{r}2.5 \\
45.8\end{array}$ & $\begin{array}{r}2.1 \\
44.3\end{array}$ & $\begin{array}{r}1.7 \\
40.7\end{array}$ & $\begin{array}{r}1.8 \\
36.8\end{array}$ & $\begin{array}{r}29.2 \\
529.4\end{array}$ & $3 . \ddot{0}$ & .671 \\
\hline & & & & & & & & & & & & & & & \\
\hline \multicolumn{16}{|l|}{ NEWAYGO } \\
\hline $\begin{array}{l}\text { Fremont } \\
\text { Hesperia }\end{array}$ & 18.5 & 17.1 & 17.7 & 16.4 & 19.1 & 31.4 & 36.1 & 23.3 & 30.3 & 33,4 & 24.4 & 18.2 & $\begin{array}{r}285.9 \\
28.0\end{array}$ & 2.866 & .151 \\
\hline Newaygo & 4.5 & 5.4 & 6.7 & 6.5 & 6.5 & 6.9 & 6.2 & 4.9 & 4.8 & 4.9 & 4.6 & 4.8 & 66.7 & .697 & .094 \\
\hline White Cloud & 9.6 & 11.2 & 12.6 & 11.0 & 11.2 & 14.0 & 15.0 & 12.3 & 11.1 & 9.9 & 9.0 & 9.2 & 136.1 & .633 & .224 \\
\hline \multicolumn{16}{|l|}{ OAKLAND } \\
\hline Holly & 14.3 & 12.9 & 14.5 & 14.9 & 15.1 & 17.2 & 16.9 & 13.9 & 14.6 & 13.3 & 12.2 & 12.8 & 172.6 & .784 & .317 \\
\hline Independence Twp. & 9.3 & 6.5 & 8.1 & 6.6 & 11.4 & 18.5 & 16.6 & 10.6 & 15.6 & 7.9 & 8.5 & 6.4 & 126.0 & $=$ & -- \\
\hline Milford & 19.0 & 18.0 & 21.5 & 20.1 & 22.4 & 27.1 & 26.3 & 21.3 & 23.0 & 20.5 & 18.2 & 18.0 & 255.4 & 1.303 & .270 \\
\hline Orion Twp. & 12.4 & 11.6 & 15.9 & 14.4 & 23.9 & 30.8 & 31.6 & 22.2 & 28.9 & 15.3 & 11.9 & 11.7 & 230.6 & 2.018 & .362 \\
\hline Oxford & 13.0 & 12.4 & 15.2 & 12.3 & 16.0 & 15.1 & 15.4 & 14.1 & 14.1 & 13.9 & 12.7 & 11.6 & 165.8 & & \\
\hline $\begin{array}{l}\text { Rochester } \\
\text { Southfield }\end{array}$ & 51.8 & 51.0 & 60.5 & 59.3 & 70.1 & 82.3 & 83.7 & 67.6 & 73.4 & 65.3 & 61.9 & 52.2 & 779.1 & 3.571 & 1.236 \\
\hline $\begin{array}{l}\text { Southfield } \\
\text { South Lyon }\end{array}$ & 59.4 & 52.7 & 61.9 & 58.1 & 70.2 & 76.7 & 65.9 & 68.0 & 63.3 & 60.7 & 54.2 & 56.7 & $\begin{array}{l}\text { e) } \\
747.1\end{array}$ & -- & -. \\
\hline Sylvan Lake & $\begin{array}{r}59.4 \\
8.1\end{array}$ & 7.1 & $\begin{array}{r}0.9 \\
5.7\end{array}$ & $\begin{array}{l}30.1 \\
6.9\end{array}$ & 9.7 & $\begin{array}{r}8.9 \\
8.9\end{array}$ & $\begin{array}{l}12.9 \\
12.4\end{array}$ & 12.8 & $\begin{array}{l}0.35 \\
10.3\end{array}$ & 5.3 & 5.2 & 5.0 & e) 97.4 & - & -. \\
\hline Walled Lake & 20.1 & 14.7 & 15.1 & 17.3 & 20.8 & 23.0 & 21.6 & 23.9 & 19.8 & 21.3 & 17.2 & 15.6 & 230.4 & -- & -- \\
\hline Waterford Twp. & 121.7 & 123.4 & 133.6 & 128.7 & 149.2 & 187.3 & 199.1 & 157.5 & 158.7 & 153.6 & 123.2 & 117.5 & $1,753.5$ & 6.422 & 3.790 \\
\hline Wolverine Lake & & & & & & & & & & & & & e) 43.6 & -- & $\cdots$ \\
\hline \multicolumn{16}{|l|}{ OCEANA } \\
\hline Hart & 5.4 & 10.6 & 20.1 & 9.7 & 32.1 & 32.5 & 45.4 & 29.7 & 27.9 & 18.3 & 34.9 & 18.1 & 284.7 & -- & -- \\
\hline Pentwater & 5.7 & 5.7 & 4.5 & 4.8 & 4.9 & 5.1 & 10.3 & 10.0 & 8.4 & 4.9 & 3.3 & 3.7 & 71.3 & .472 & -- \\
\hline Shelby & 10.4 & 9.6 & 10.2 & 9.9 & 11.4 & 16.6 & 19.2 & 12.2 & 11.8 & 9.5 & 9.4 & 9.1 & 139.3 & -- & $-\cdot$ \\
\hline \multicolumn{16}{|l|}{ OGEMAW } \\
\hline West Branch & 10.1 & 9.6 & 11.3 & 9.8 & 10.7 & 12.2 & 13.9 & 12.5 & 12.3 & 10.6 & 8.7 & 9.1 & 130.8 & .702 & .166 \\
\hline \multirow{2}{*}{\multicolumn{16}{|c|}{$\begin{array}{l}\text { ONTONAGON } \\
\text { Bergland Twp. }\end{array}$}} \\
\hline & & & & & & & & & & & & & e) 12.7 & .094 & .026 \\
\hline \multicolumn{16}{|l|}{ OSCEOLA } \\
\hline $\begin{array}{l}\text { Evart } \\
\text { Marion }\end{array}$ & $\begin{array}{r}73.2 \\
7.6\end{array}$ & $\begin{array}{r}58.6 \\
6.1\end{array}$ & $\begin{array}{r}70.0 \\
7.4\end{array}$ & $\begin{array}{r}79.2 \\
6.4\end{array}$ & $\begin{array}{r}84.0 \\
6.6\end{array}$ & $\begin{array}{r}71.1 \\
3.9\end{array}$ & $\begin{array}{r}75.0 \\
7.1\end{array}$ & $\begin{array}{r}67.0 \\
4.0\end{array}$ & $\begin{array}{r}54.0 \\
4.2\end{array}$ & $\begin{array}{r}60.3 \\
4.0\end{array}$ & $\begin{array}{r}55.1 \\
4.1\end{array}$ & $\begin{array}{r}40.0 \\
4.1\end{array}$ & $\begin{array}{r}787.5 \\
65.5\end{array}$ & $\begin{array}{r}3.222 \\
.278\end{array}$ & $\begin{array}{l}.820 \\
.119\end{array}$ \\
\hline Reed City & 14.6 & 13.1 & 13.5 & 13.0 & 16.2 & 17.3 & 17.5 & 14.1 & 12.8 & 14.9 & 11.4 & 11.6 & 170.0 & - & - \\
\hline \multicolumn{16}{|l|}{ OTSEGO } \\
\hline Gaylord & 15.7 & 15.8 & 18.3 & 19.1 & 21.6 & 31.2 & 25.4 & 21.7 & 15.6 & 16.8 & 16.8 & 14.8 & 232.8 & $\ddot{r e c}$ & $-\cdots$ \\
\hline Alpine Center, Gaylord & 1.1 & 1.0 & 1.0 & 1.1 & 1.2 & 1.1 & 1.2 & 1.3 & 1.2 & 1.3 & 1.2 & 1.1 & 13.8 & .055 & .020 \\
\hline \multicolumn{16}{|l|}{ OTTANA } \\
\hline Spring Lake & 10.2 & 10.5 & 10.4 & 10.7 & 14.3 & 21.8 & 25.2 & 15.7 & 16.2 & 11.4 & 10.2 & 9.8 & 166.4 & 1.600 & .183 \\
\hline \multicolumn{16}{|l|}{ PRESQUE ISLE } \\
\hline Onaway & 5.1 & 6.0 & 6.0 & 5.1 & .4 .3 & 4.7 & 5.5 & 4.3 & 3.5 & 4.3 & 7.5 & 4.4 & 60.7 & .210 & -- \\
\hline Rogers City & 11.7 & 10.9 & 12.6 & 8.2 & 10.3 & 11.3 & 15.7 & 9.6 & 10.8 & 9.6 & 9.9 & 10.8 & 131.4 & & $-\cdot$ \\
\hline \multicolumn{16}{|l|}{ ROSCOMMON } \\
\hline Roscommon & 5.7 & 4.9 & 5.6 & 3.7 & 5.0 & 7.0 & 8.2 & 7.5 & 4.9 & 4.9 & 4.8 & 4.4 & 66.6 & -- & -- \\
\hline \multicolumn{16}{|l|}{ SAGINAW } \\
\hline $\begin{array}{l}\text { Chesaning } \\
\text { Tittabawassee Twp. }\end{array}$ & 9.6 & $\begin{array}{l}9.9 \\
4.6\end{array}$ & $\begin{array}{r}11.8 \\
5.3\end{array}$ & $\begin{array}{r}11.9 \\
5.6\end{array}$ & $\begin{array}{r}12.1 \\
6.6\end{array}$ & $\begin{array}{r}11.8 \\
7.4\end{array}$ & $\begin{array}{r}10.5 \\
7.1\end{array}$ & $\begin{array}{r}11.1 \\
6.1\end{array}$ & $\begin{array}{r}10.6 \\
6.3\end{array}$ & $\begin{array}{l}7.9 \\
6.1\end{array}$ & $\begin{array}{l}7.8 \\
5.3\end{array}$ & $\begin{array}{l}7.6 \\
5.3\end{array}$ & $\begin{array}{r}122.6 \\
70.1\end{array}$ & $\begin{array}{l}.526 \\
.338\end{array}$ & $\begin{array}{l}.114 \\
.150\end{array}$ \\
\hline \multicolumn{16}{|l|}{ ST. CLAIR } \\
\hline Capac & 4.2 & 4.0 & 4.0 & 3.6 & 4.4 & 5.1 & 4.6 & 5.4 & 5.2 & 5.0 & 4.9 & 5.1 & 55.5 & .371 & .093 \\
\hline Yale & 5.5 & 6.8 & 4.5 & 5.0 & 6.5 & 6.1 & 5.4 & 5.6 & 6.5 & 6.2 & 7.0 & 5.9 & 71.0 & .649 & .016 \\
\hline
\end{tabular}


TABLE 2. REPORTED GROUND-WATER PUMPAGE, IN 1979. (IN MILLIONS OF GALLONS)-CONTINUED

\begin{tabular}{|c|c|c|c|c|c|c|c|c|c|c|c|c|c|c|c|}
\hline $\begin{array}{c}\text { COUNTY } \\
\text { AND } \\
\text { WATER USER }\end{array}$ & JAN & FEB & MAR & APR & MAY & JUN & JUL & AUG & SEP & OCT & NOV & $\mathrm{DEC}$ & $\begin{array}{l}1979 \\
\text { TOTAL }\end{array}$ & $\begin{array}{l}\text { MAX } \\
\text { DAY }\end{array}$ & $\begin{array}{l}\text { MIN } \\
\text { DAY }\end{array}$ \\
\hline \multicolumn{16}{|l|}{ ST. JOSEPH } \\
\hline Constantine & 10.7 & 10.0 & 10.5 & 7.3 & 7.9 & 8.8 & 8.9 & 7.5 & 7.3 & 7.5 & 6.6 & 7.9 & 100.9 & .539 & .112 \\
\hline Mendon & 11.8 & 6.5 & 7.3 & 11.8 & 13.6 & 13.0 & 13.4 & 14.5 & 12.5 & 13.0 & 9.4 & 7.6 & 134.4 & .848 & .086 \\
\hline Sturgis & 65.1 & 73.9 & 88.2 & 67.4 & 74.8 & 85.3 & 89.0 & 81.6 & 81.2 & 79.6 & 72.9 & 68.3 & e) 927.3 & 3.968 & 1.498 \\
\hline Three Rivers & 34.7 & 33.3 & 38.3 & 34.1 & 42.0 & 42.5 & 45.2 & 37.0 & 35.6 & 23.1 & 26.0 & 23.8 & 415.6 & -- & - \\
\hline \multicolumn{16}{|l|}{ SANILAC } \\
\hline Brown City & 4.3 & 4.1 & 4.3 & 4.0 & 4.3 & 5.3 & 4.7 & 4.1 & 4.5 & 3.5 & 3.5 & 3.4 & 50.0 & -- & -- \\
\hline Croswell & 10.9 & 12.4 & 13.0 & 13.2 & 18.1 & 17.5 & 20.3 & 30.0 & 18.4 & 19.3 & 15.7 & 16.3 & 205.1 & 1.033 & .200 \\
\hline Deckerville & 3.4 & 3.2 & 3.2 & 2.8 & 3.4 & 4.1 & 3.9 & 4.5 & 3.6 & 3.3 & 2.9 & 2.6 & 40.9 & .202 & .039 \\
\hline Marlette & 9.3 & 8.7 & 9.7 & 9.3 & 10.6 & 11.5 & 11.6 & 11.6 & 11.2 & 12.3 & 10.5 & 8.5 & 124.8 & .502 & .142 \\
\hline Peck & 1.9 & 2.0 & 2.3 & 1.8 & 3.0 & 1.9 & 2.5 & 2.4 & 1.9 & 2.0 & 1.6 & 1.7 & 25.0 & -- & -- \\
\hline Port Sanilac & 3.1 & 3.3 & 2.7 & 3.9 & 4.7 & 5.2 & 6.1 & 5.7 & 3.2 & 4.0 & 4.8 & 3.0 & 49.7 & .258 & .072 \\
\hline Sandusky & 13.4 & 17.2 & 18.5 & 14.7 & 16.7 & 18.5 & 17.7 & 17.5 & 18.9 & 18.4 & 15.0 & 14.0 & 200.5 & .968 & .148 \\
\hline \multicolumn{16}{|l|}{ SHIAWASSEE } \\
\hline Bancroft & 1.5 & 1.3 & 1.2 & 1.2 & 1.1 & 2.1 & 1.9 & 1.9 & 1.7 & 1.5 & 1.4 & 1.3 & 18.1 & -- & -- \\
\hline Byron & 1.8 & 1.9 & 1.7 & 1.6 & 2.0 & 2.3 & 3.4 & 2.7 & 2.6 & 2.0 & 1.8 & 1.9 & 25.7 & -- & -- \\
\hline Durand & 10.7 & 9.7 & 10.2 & 10.2 & 11.4 & 12.6 & 12.0 & 13.4 & 11.2 & 10.4 & 9.5 & 9.8 & 131.1 & .515 & .180 \\
\hline Owosso & 73.6 & 72.3 & 75.4 & 71.5 & 79.7 & 84.3 & 86.4 & 78.1 & 77.6 & 79.3 & 73.1 & 73.2 & e) 924.5 & 3.504 & 1.353 \\
\hline Perry & 4.9 & 5.0 & 4.9 & 4.4 & 5.3 & 6.4 & 6.7 & 5.1 & 5.5 & 4.6 & 4.4 & 5.0 & 62.2 & .364 & .120 \\
\hline \multicolumn{16}{|l|}{ TUSCOLA } \\
\hline Akron & 9.5 & 10.9 & 8.8 & 10.5 & 13.4 & 8.9 & 9.8 & 13.0 & 10.9 & 4.6 & 6.1 & 4.6 & 111.0 & -. & -- \\
\hline Caro & 21.4 & 16.8 & 15.0 & 14.0 & 16.1 & 18.2 & 18.2 & 17.7 & 17.1 & 21.1 & 19.9 & 19.5 & 215.0 & -- & -- \\
\hline Cass City & 9.8 & 8.5 & 10.1 & 9.7 & 11.0 & 11.2 & 12.3 & 10.3 & 9.6 & 7.9 & 7.4 & 7.2 & 115.0 & .568 & .160 \\
\hline Kingston & 1.3 & 1.4 & 1.4 & 1.3 & 1.3 & 2.1 & 2.8 & 1.4 & 1.2 & .9 & 1.0 & 1.2 & 17.3 & .147 & .013 \\
\hline Mayville & 3.4 & 4.4 & 3.2 & 3.1 & 4.5 & 4.1 & 3.4 & 3.8 & 3.5 & 4.3 & 2.9 & 3.3 & 43.9 & -. & -. \\
\hline State Hosp., Caro & 4.5 & 4.7 & 3.9 & 3.6 & 4.0 & 4.0 & 4.3 & 4.1 & 4.0 & 4.3 & 4.2 & 4.5 & 50.1 & .280 & .070 \\
\hline Vassar & 19.4 & 13.6 & 15.3 & 13.7 & 15.8 & 16.8 & 30.8 & 26.7 & 24.9 & 21.9 & 19.6 & 18.1 & 236.6 & 3.298 & .379 \\
\hline \multicolumn{16}{|l|}{ VAN BUREN } \\
\hline Bangor & 8.6 & 7.9 & 8.4 & 7.1 & 5.7 & 5.7 & 7.0 & 5.8 & 5.4 & 5.8 & 5.0 & 4.7 & 77.1 & .538 & .086 \\
\hline Decatur & 9.9 & 10.2 & 11.8 & 12.9 & 12.2 & 9.7 & 11.4 & 8.8 & 9.9 & 10.2 & 8.8 & 7.5 & 123.3 & -- & -- \\
\hline Gobles & 1.6 & 1.6 & 2.2 & 3.0 & 2.3 & 4.3 & 3.0 & 2.6 & 2.1 & 2.0 & 1.7 & 1.2 & 27.6 & .278 & -- \\
\hline Hartford & 9.1 & 8.3 & 7.8 & 8.4 & 10.6 & 16.6 & 9.8 & 9.2 & 6.3 & 7.5 & 6.9 & 6.9 & 107.4 & -- & .111 \\
\hline Lawrence & 3.8 & 2.2 & 1.8 & 1.6 & 1.7 & 1.8 & 2.0 & 2.1 & 3.2 & 3.3 & 3.7 & 3.2 & 30.4 & -- & -- \\
\hline Lawton & 21.5 & 23.0 & 22.6 & 20.2 & 27.1 & 24.9 & 27.1 & 26.6 & 32.0 & 43.3 & 14.8 & 12.3 & 295.4 & 1.800 & .176 \\
\hline Paw Paw & 13.0 & 14.2 & 14.7 & 14.5 & 14.5 & 16.4 & 22.0 & 18.8 & 19.6 & 16.3 & 17.5 & 17.8 & 199.3 & 1.203 & .004 \\
\hline \multicolumn{16}{|l|}{ WASHTENAW } \\
\hline Ann Arbor & 33.0 & 19.0 & 19.0 & 0.0 & 8.0 & 35.0 & 29.0 & 38.0 & 36.0 & 83.0 & 37.0 & 35.0 & g) 372.0 & -- & -- \\
\hline Chelsea & 17.7 & 15.5 & 16.7 & 14.6 & 16.5 & 19.2 & 17.9 & 18.2 & 17.6 & 17.4 & 15.5 & 15.6 & 202.4 & .911 & .309 \\
\hline Dexter & 6.1 & 5.4 & 6.2 & 6.0 & 6.3 & 6.1 & 6.8 & 6.8 & 6.6 & 6.2 & 6.4 & 6.5 & 75.4 & -- & -- \\
\hline Manchester & 6.9 & 7.6 & 7.1 & 6.3 & 7.4 & 8.7 & 8.1 & 7.4 & 7.6 & 6.6 & 5.8 & 6.2 & 85.7 & .443 & .120 \\
\hline Saline & 38.5 & 33.5 & 38.5 & 37.9 & 40.6 & 45.1 & 38.7 & 39.2 & 39.8 & 35.5 & 31.2 & 26.1 & 444.6 & 2.076 & .575 \\
\hline Webster Twp. & 2.1 & 1.9 & 2.0 & 2.1 & 3.5 & 6.0 & 4.3 & 3.1 & 5.0 & 2.4 & 2.1 & 2.4 & 36.9 & .436 & .035 \\
\hline Ypsilanti & 118.4 & 108.1 & 121.2 & 80.7 & 95.7 & 116.1 & 112.4 & 96.9 & 78.2 & 60.2 & 64.0 & 87.6 & $1,139.5$ & 5.208 & 1.471 \\
\hline Ypsilanti Twp. & 0 & 0 & 0 & 0 & 2.0 & 87.2 & 79.9 & 41.7 & 2.2 & 35.0 & 91.9 & 90.8 & 430.7 & 8.570 & 0 \\
\hline \multicolumn{16}{|l|}{ WEXFORD } \\
\hline Cadillac & 62.7 & 59.1 & 63.8 & 52.1 & 61.7 & 85.4 & 104.1 & 70.5 & 75.3 & 79.6 & 52.8 & 51.6 & 798.7 & 4.497 & 1.049 \\
\hline Manton & 5.8 & 7.7 & 8.2 & 6.7 & 8.0 & 7.7 & 8.9 & 7.6 & 7.1 & 5.9 & 4.5 & 4.0 & 82.1 & .325 & -- \\
\hline
\end{tabular}

NOTES

a) Amount pumped to supply Houghton, Hancock, Portage Township, Copper Range Company,

b) Amount pumped to supply Painesdale, Trimountain, Baltic, and South Range.

c) Amount Pumped to supply Calumet, Calumet Township, Copper City, Lake Linden, Laurim,

Osceola Township, Torch Lake Township, Ahmeek, and Alleouez Township.

d) Use Ranney collector system at Chippewa River site.

e) Wholly or partly estimated.

f) Converted to City of Kalamazoo water supply.

g) Also pumped 5,044 million gallons from Huron River. 
LOCAL IDENTIFIER: See section in text entitled "Well-numbering system"; a1so includes abbreviated spelling of county name. GEOLOGIC UNIT: 112GLCL Glacial deposits 112SDGV Sand and grave1 344DUND Dundee Formation

UNITS: Units are reported in NTU $=$ Nephelometric Turbidity Units; MG $/ \mathrm{L}=$ Milligrams per Liter; UG/L $=$ Micrograms per Liter

$39 \mathrm{~N} \mathrm{O3W} \mathrm{29CBCBOI} \mathrm{CHBYGN}$ $39 \mathrm{~N} 03 \mathrm{~W} 29 \mathrm{CBCBO2}$ CHBYGN 075 O2W 15BCBAOI HILSDL 2IN 13W 19BDOCOI MANSTE 075 O6E 15ACAADI MONROE

$11 N$ OBW IODBB OI MNTCLM 3IN O2E O3ADD OI MNTMRY $14 \mathrm{~N} 13 \mathrm{~W} \mathrm{30DC}$ OI NEWYGO I5N $13 W 2$ 20DDB 01 NEWYGO $25 \mathrm{~N}$ O2E 14DOA OI OSCODA

$30 N$ O3W 19ABBBOI OTSEGO $34 \mathrm{~N}$ OTE $270 \mathrm{~A}$ OI PRSO $22 N$ I $12 W 13 B B$ OI WEXFRD

$\begin{array}{cr}\text { DATE } & \text { GEO- } \\ \text { OF } & \text { LOGIC } \\ \text { SAMPLE } & \text { UNIT }\end{array}$

DEPTH
OF TEMPERWELL, ATURE, TOTAL
(FEET)
(DEG C) BID(NTU)

79-08-23 3440UND 79-08-23 $11250 G V$ 79-08-20 112SAND 79-08-22 1125AND 79-08-09 348DRRV

79-08-21 11250GV 79-08-23 112GLCL 79-08-21 $112 \mathrm{GLCL}$ 79-00-21 $1125 A N D$ $79-08-24 \quad 112 \mathrm{GLCL}$

79-08-23 1125AND $79-08-23$ 341TRVR 79-08-22 II2SAND
125
55
150
46
72
66
121
60
45
48
95
91
447

$\begin{array}{rr}8.5 & 3.0 \\ 8.5 & 2.0 \\ 10.5 & 15 \\ 8.5 & .00\end{array}$

10.5

1.00

$9.0 \quad 1.0$

$\begin{array}{rr}8.5 & 25 \\ 10.0 & 5.0\end{array}$

$8.5 \quad 2.0$

7.58 .0

7.5
9.0

8.0
9.0
SPE-

IPLAT- DUCT-

INUM ANCE

COBALT (MICRO-

UNITS)

MHOS)

DIOXIDE ALKA-

DIS- LINITY

(MG/L

(UNITS) AS CO2) CACO3)

$\begin{array}{rr}85 & 268 \\ 100 & 2 \\ 3 & 5 \\ 1 & 2 \\ 3 & \end{array}$

$\begin{array}{llll}268 & 7.5 & 9.1 & 148\end{array}$

$\begin{array}{llll}595 & 7.5 & 8.6 & 139 \\ 7.4 & 23 & 295\end{array}$

$246 \quad 8.0 \quad 2.4 \quad 123$

$\begin{array}{lll}456 & 7.8 & 6.3\end{array}$

417

142

$\begin{array}{lll}7.8 & 6.3 & 205 \\ 7.7 & 7.7 & 197\end{array}$

$\begin{array}{rrr}7.6 & 8.8 & 180 \\ 8.6 & .3 & 56\end{array}$

7.510

$\begin{array}{lll}7.6 & 8.8 & 180 \\ 7.6 & 10 & 205\end{array}$

400

\section{LOCAL
IDENT- \\ I-}

$39 \mathrm{~N} 03 W$ 29CBCB01 CHBYGN $39 \mathrm{~N}$ 03W 29CBCB02 CHBYGN 075 02W 15BCBAOI HILSDL 2IN I3W I9BDDCOI MANSTE 075 O6E ISACAAOI MONROE

$11 N$ OBW $1008 B$ OI MNTCLM 3 IN O2E O3ADO OI MNTMR $14 \mathrm{~N} 13 W 300 \mathrm{~W}$ OI NEWYGO $15 \mathrm{~N} \mathrm{13W} 2000 B$ OI NEWYGO 25N O2E 14DOA 01 OSCODA

$30 N$ 03W 19ABBBOI OTSEGO $34 \mathrm{~N}$ 07E 27DA 01 PRSQ $22 \mathrm{~N} 12 W 13 B B$ 01 WEXFRD

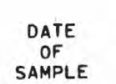

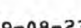

$79-08-23$
$79-08-23$

$79-08-23$
$79-08-20$ $79-08-20$
$79-08-22$ $79-08-22$
$79-08-09$

$79-08-21$

$79-08-23$ $79-08-2$ $79-08-21$

$79-08-23$ $79-08-23$ $79-08-22$

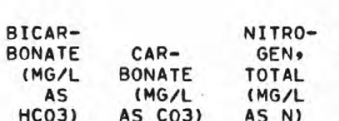

GEN, GEN, GEN, TOTAL (MG/L

AS N)

180
170
360
150
250
250
240
220
64
200
220
250
190

180
170

$\begin{array}{ll}.47 & .31 \\ .45 & .25 \\ .29 & .15 \\ .26 & .14 \\ 4.8 & 4.6\end{array}$

.86

1.12

1.99
.09

.04

2.9

.50
.24
.01
.04
.06
.34

.34
.99

NITRO-
GEN,
AMMONIA
TOTAL
(MG/L
AS N)

NITRONTRITE NITRATE

TOTAL

AS N)

.15
.17
.14
.04
.25
.00
.01
.76
.00
.00

.16
.01
.00
.00
.00
.00

.00

.00
.00

.00

.00
.00

.0

.00
.00
NITRO-

GEN, AM- NITROMONIA - GEN, ORGANIC NO2+NO TOTAL TOTAL AS N) AS N)

$\begin{array}{lcc}.00 & .46 & .01 \\ .03 & .42 & .03 \\ .00 & .29 & .00 \\ .08 & .18 & .08 \\ .00 & 4.8 & .00 \\ .82 & .04 & .82 \\ .05 & .07 & .05 \\ .00 & 1.1 & .00 \\ .00 & .99 & .00 \\ 2.9 & .01 & 2.9 \\ .34 & .16 & .34 \\ .00 & .24 & .00 \\ .00 & .01 & .00\end{array}$

LOCAL
IDENT-
I-
FIER

$39 \mathrm{~N} \mathrm{03W} \mathrm{29CBCBO1} \mathrm{CHBYGN}$ $39 \mathrm{~N} 03 W 29 \mathrm{CBCBO2}$ CHBYGN D7S 02W 15BCBAOI HILSDL 2IN 13W 19BDDCOI MANSTE O7S O6E ISACAAOI MONROE

$11 N$ 08W 10088 O1 MNTCLM 3IN OZE O3ADD OI MNTMRY $\begin{array}{lllll}31 N & 02 E & 03 A D D & 01 & \text { MNTMRY } \\ 14 \mathrm{~N} & 13 \mathrm{~W} & 300 \mathrm{C} \text { OI } & \text { NEWYGO }\end{array}$ $15 \mathrm{~N}$ 13W $2000 \mathrm{~W}$ OI NEWYGO $25 \mathrm{~N}$ O2E $1400 A$ OI OSCODA

3ON O3W 19ABBBOI OTSEGO $34 \mathrm{~N}$ 07E 27DA 01 PRSQ I $22 \mathrm{~N} 12 \mathrm{~W} 13 \mathrm{BB}$ O1 WEXFRD

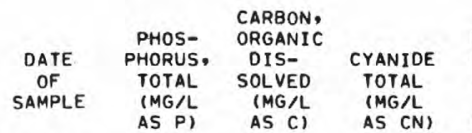

HARD-
NESS
(MG/L
AS
CACO3)

HARDNESS, CALCIUM NONCARBONATE (MG/L (ACO3) DISSOLVED (MG/L
AS CA)

MAGNE-

SIUM, SODIUM, SODIUM

OIS- DIS- AD-

DIS- DIS- SORP-

SOLVED

AS MG) AS NA)

TION
RATIO

$\begin{array}{ll}79-08-23 & .010 \\ 79-08-23 & .010\end{array}$

$79-08-23$

$79-08-20$

$79-08-22$

$79-08-09$

11.8

.00

140

130
310

.010
.000

.000

.00
1.00

.00
.00

10

6.7
24
8.7

18.7

$\begin{array}{rr}2.2 & .1 \\ 5.5 & .2 \\ 7.8 & .2 \\ 23^{.8} & .0\end{array}$

$79-08-21$

.000

$-23$

$79-08-21$

.000
.060

.060
.070

160

$.00+240$

$\begin{array}{ll}-5 & .00\end{array}$

240
220

170

$79-08-24$

.000

2.7

.00

62
190

79-08-23

$79-08-2$

.000
.010

.010
.010

2.9

.00

.00
.00

200

400
160

20

20
16
16

16.3

2.9
2.5

2.0
9.0
3.0

.1

10

20
190

$40^{7.9}$

5.4
64
2.6

.2
1.4
.1 
TABLE 3. WATER-QUALITY DATA. (CONTINUED)
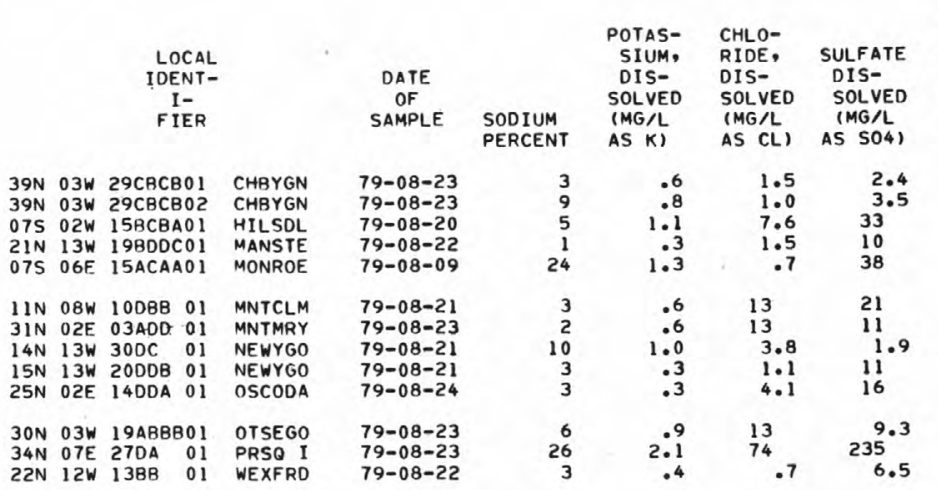

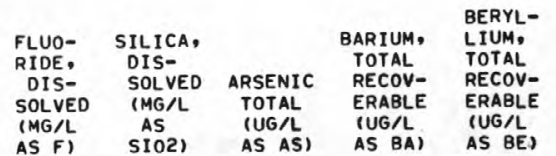

$79-08-23$

$79-08-23$

79-08-20

$79-08-22$

3

6

$$
\begin{aligned}
& 1.5 \\
& 1.0 \\
& 7.6 \\
& 1.5
\end{aligned}
$$

2.4
3.5
33
10
38

$\begin{array}{ll}.1 & 11 \\ .1 & 10 \\ .0 & 14 \\ 0 & 6.7\end{array}$

AS BE

79-08-21

79-08-23

79-08-21

$79-08-21$
$79-08-24$

$\begin{array}{rr}3 & .6 \\ 9 & .8 \\ 5 & 1.1 \\ 1 & .3 \\ 24 & 1.3\end{array}$

1.5
.7

$\begin{array}{lll}.6 & 13 & 21 \\ .6 & 13 & 11 \\ 1.0 & 3.8 & 1.9 \\ .3 & 1.1 & 11 \\ .3 & 4.1 & 16\end{array}$

.1
$: 1$
-0
.0

11
10
14
5.4
7.7

$79-08-23$

79-08-23

79-08-22

$\begin{array}{ccc}.9 & 13 & 9.3 \\ 2.1 & 74 & 235 \\ .4 & .7 & 6.5\end{array}$

.0
1.4
.1

6.4
8.8
9.8

\begin{tabular}{|c|c|c|}
\hline 0 & & 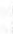 \\
\hline 100 & & 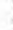 \\
\hline & & 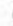 \\
\hline 100 & & \\
\hline 0 & & \\
\hline 0 & & \\
\hline 0 & & \\
\hline 0 & & \\
\hline 0 & & \\
\hline $\begin{array}{l}0 \\
0\end{array}$ & $\bullet$ & \\
\hline 100 & & \\
\hline
\end{tabular}
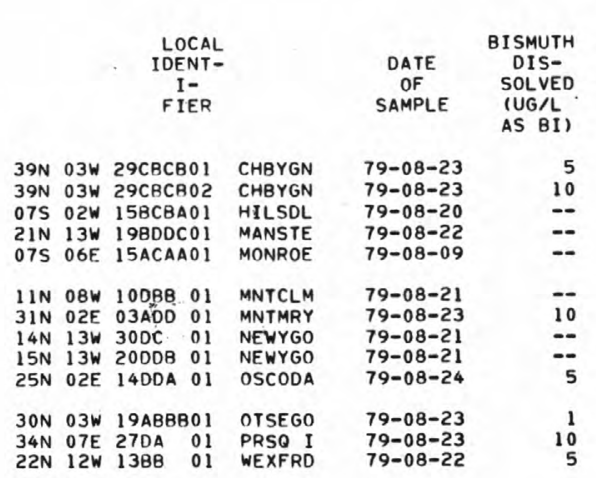

BORON, CAO
TOTAL
RECOV-
ERABLE

UG儿

AS B)

79-08-23

79-08-23

79-08-20

$79-08-22$

79-08-09

$79-08-21$
$79-08-23$

$79-08-23$
$79-08-21$

$79-08-21$

79-08-24

79-08-23

$79-08-23$
$79-08-22$
5

5
10

\begin{abstract}
20
\end{abstract}
10
80

80
10
40

a

1
1
0
0
0

$-$

$\frac{10}{--}$

40

10
30
10
10

1
10
5

20
60
CADMIUM

TOTAL

RECOV-

AS CD)

CHRO-

MIUM, COBALT, COPPER, IRON,

TOTAL

ERABLE

IUG/L

AS (R)

TOTAL TOTAL

$\begin{array}{ll}\text { RECOV- } & \text { RECOV- } \\ \text { ERABLE } & \text { ERABLE }\end{array}$

IUG/L

ERABLE
(UG/L

TOTAL

RECOV-

(UG/L

AS FE)

IRON,

DIS-

UG/L (UG/L

$\begin{array}{rrr}2400 & 2400 & 7 \\ 3100 & 3200 & 8 \\ 1200 & 1300 & 3 \\ 70 & 30 & 2 \\ 120 & 60 & 3\end{array}$

$\begin{array}{rr}3 & 10 \\ 2 & 10 \\ 0 & 10 \\ 0 & 10 \\ 1 & 10\end{array}$

10
10
10
20
20
10
10
10
10
10$$
\begin{aligned}
& 3 \\
& 4 \\
& 0 \\
& 1 \\
& 0 \\
& 1 \\
& 0 \\
& 1 \\
& 1 \\
& 3
\end{aligned}
$$

$\begin{array}{rr}2 & 2400 \\ 4 & 3100 \\ 2 & 1200 \\ 10 & 70 \\ 3 & 120\end{array}$

240

1200

1000
620

20
50

840

760
1000

10
10
$<10$

0
3
3

720

MANGA- MANGA-

NESE

PENDED

RECOV.

(UG/L

AS MN)

NESE,

TOTAL

RECOV-

ERABLE

(US MN)

MANGA- DENUM, NICKEL, SILVER,

MANGA-

NESE,

SOLVED

(UG/L

TOTAL

RECOV

RECOV- RECOV-

ERABLE ERABLE

AS MOI IUG/L

AS NI)

SILVER,
TOTAL

TOTAL

ERABLE

(UG/L

AS AG)

STRON-

TIUM,

TOTAL

RECOV-

ERABLE

(UG/L.

AS SR)

VANA- ZINC,

DIUM, TOTAL

DIS- RECOV-

SOLVED ERABLE

AS VI AS ZN)

$39 N$ 03W 29CBCBOI CHBYGN 39N 03W 29CBCB02 CHBYGN

07S 02W 15BCBA01 HILSOL

2IN 13W 19BDDCOI MANSTE

79-08-23

$79-08-23$

$79-08-20$

$79-08-22$

90
70
30
60
20

90
60
30
60
9

1
1
14
0
3

5
7
0
1
2

100
40
520
190

190
39000

$\begin{array}{rr}.0 & 10 \\ 0 & 10 \\ -0 & 110 \\ -- & 0\end{array}$

$79-08-21$

$79-08-23$

$79-08-21$

$79-08-21$
$79-08-24$

79-08-23

$79-08-23$
$79-08-23$

$79-08-23$
$79-08-22$
150
130
1200
90

70
2500
.010

$34 \mathrm{~N}$ OTE $270 \mathrm{~A} 01$ PRSQ

$\begin{array}{lllll}34 \mathrm{~N} & 07 E & 270 A & 01 & P R S Q \\ 22 \mathrm{~N} & 12 \mathrm{~W} & 13 \mathrm{BB} & 01 & \text { WEXFRD }\end{array}$

20 
TABLE 3. WATER-QUALITY DATA. (CONTINUED)

\begin{tabular}{|c|c|c|c|c|c|c|c|c|c|c|c|c|}
\hline & $\begin{array}{l}\text { LOCALL } \\
\text { IDENT- } \\
\text { I- } \\
\text { FIER }\end{array}$ & & $\begin{array}{c}\text { DATE } \\
\text { OF } \\
\text { SAMPLE }\end{array}$ & $\begin{array}{l}\text { TIN. } \\
\text { DIS- } \\
\text { SOLVED } \\
\text { IUGA } \\
\text { AS SN } \\
\text { (A.A.S. } \\
\text { DIRECT) }\end{array}$ & $\begin{array}{l}\text { ALUM- } \\
\text { INUM } \\
\text { TOTAL } \\
\text { RECOV- } \\
\text { ERABE } \\
\text { CUGLL } \\
\text { AS ALI }\end{array}$ & $\begin{array}{l}\text { GALLIUM } \\
\text { DIS- } \\
\text { SOLVED } \\
\text { IUG } \\
\text { AS GA }\end{array}$ & $\begin{array}{l}\text { GER- } \\
\text { MANIUM, } \\
\text { DIS- } \\
\text { SOLVED } \\
\text { UUG/L } \\
\text { AS GE) }\end{array}$ & $\begin{array}{l}\text { LITHIUM } \\
\text { TOTAL } \\
\text { RECOV- } \\
\text { ERABLE } \\
\text { IUGA } \\
\text { AS LI }\end{array}$ & $\begin{array}{l}\text { SELE- } \\
\text { NIUM, } \\
\text { TOTAL } \\
\text { (UGL } \\
\text { AS SE) }\end{array}$ & $\begin{array}{l}\text { TI- } \\
\text { TANIUM, } \\
\text { DIS- } \\
\text { SOLVED } \\
\text { CUG } \\
\text { AS TII }\end{array}$ & $\begin{array}{l}\text { ZIR- } \\
\text { CONIUM, } \\
\text { DIS- } \\
\text { SOLVED } \\
\text { CUG/L } \\
\text { AS ZR) }\end{array}$ & $\begin{array}{c}\text { PHENOLS } \\
\text { (UG/L) }\end{array}$ \\
\hline $\begin{array}{ll}39 N & 03 W \\
39 N & 03 W \\
075 & 02 W \\
21 N & 13 W \\
075 & 06 E\end{array}$ & $\begin{array}{l}29 C B C B 01 \\
29 C B C B 02 \\
15 B C B A 01 \\
19 B D D C 01 \\
15 A C A A 01\end{array}$ & $\begin{array}{l}\text { CHBYGN } \\
\text { CHBGN } \\
\text { HILSDL } \\
\text { MANSEE } \\
\text { MONROE }\end{array}$ & $\begin{array}{l}79-08-23 \\
79-08-23 \\
79-08-20 \\
79-08-22 \\
79-08-09\end{array}$ & $\begin{array}{l}0 \\
0 \\
-\because \\
--\end{array}$ & $\begin{array}{r}300 \\
150 \\
40 \\
50 \\
30\end{array}$ & $\begin{array}{l}0 \\
0 \\
\because- \\
--\end{array}$ & \begin{tabular}{l}
0 \\
$\therefore$ \\
\hdashline- \\
--
\end{tabular} & $\begin{array}{l}0 \\
0 \\
0 \\
0 \\
0\end{array}$ & $\begin{array}{l}0 \\
0 \\
0 \\
0 \\
0\end{array}$ & $\begin{array}{r}0 \\
0 \\
-- \\
=-\end{array}$ & $\begin{array}{l}0 \\
\therefore \\
\because-\end{array}$ & 0 \\
\hline 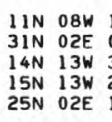 & $\begin{array}{ll}1008 B & 01 \\
03 A 00 & 01 \\
300 C & 01 \\
2000 B & 01 \\
1400 A & 01\end{array}$ & $\begin{array}{l}\text { MNTCLM } \\
\text { MNTMRY } \\
\text { NEWGGO } \\
\text { NEWGGO } \\
\text { OSCODA }\end{array}$ & $79-08-21$ & $\begin{array}{l}-\ddot{0} \\
\ddot{0}\end{array}$ & $\begin{array}{l}50 \\
70 \\
90 \\
50 \\
50\end{array}$ & \begin{tabular}{l}
-- \\
-0 \\
\hdashline 0
\end{tabular} & $\begin{array}{l}-- \\
-0 \\
-0\end{array}$ & $\begin{array}{l}0 \\
0 \\
0 \\
0 \\
0\end{array}$ & $\begin{array}{l}0 \\
0 \\
0 \\
0 \\
0\end{array}$ & $\begin{array}{l}-\ddot{0} \\
\ddot{0}\end{array}$ & $\begin{array}{l}-\ddot{0} \\
\because \ddot{0}\end{array}$ & 0 \\
\hline 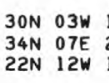 & $\begin{array}{ll}19 A B B D & 1 \\
27 D A & 01 \\
13 B B & 01\end{array}$ & $\begin{array}{l}\text { OTSEGO } \\
\text { PRSQ I } \\
\text { WEXFRD }\end{array}$ & & $\begin{array}{l}0 \\
1 \\
0\end{array}$ & $\begin{array}{l}60 \\
170 \\
130\end{array}$ & $\begin{array}{l}0 \\
0 \\
0\end{array}$ & $\begin{array}{l}0 \\
0 \\
0\end{array}$ & $\begin{array}{l}0 \\
0 \\
0\end{array}$ & $\begin{array}{l}0 \\
0 \\
0\end{array}$ & $\begin{array}{l}0 \\
0 \\
0\end{array}$ & $\begin{array}{l}0 \\
0 \\
0\end{array}$ & \\
\hline & $\begin{array}{l}\text { LOCAL } \\
\text { IDENT- } \\
\text { I- } \\
\text { FIER }\end{array}$ & & $\begin{array}{c}\text { DATE } \\
\text { OF } \\
\text { SAMPLE }\end{array}$ & $\begin{array}{l}\text { NAPH- } \\
\text { THA- } \\
\text { LENES, } \\
\text { POLY } \\
\text { CHLOR. } \\
\text { TOTAL } \\
\text { (UGL }\end{array}$ & $\begin{array}{l}\text { ALDRIN, } \\
\text { OTIAL } \\
\text { (UG/L) }\end{array}$ & $\begin{array}{l}\text { LINDANE } \\
\text { OTALAL } \\
\text { (UG/L) }\end{array}$ & $\begin{array}{l}\text { CHLOR- } \\
\text { DANE, } \\
\text { TOAAL } \\
\text { (UG/L) }\end{array}$ & $\begin{array}{l}\text { DOD, } \\
\text { TOTAL } \\
\text { (UGLL) }\end{array}$ & $\begin{array}{l}\text { DOE, } \\
\text { TOTAL } \\
\text { (UG/L) }\end{array}$ & $\begin{array}{l}\text { DDT, } \\
\text { TOTAL } \\
\text { (UGLL) }\end{array}$ & $\begin{array}{l}\text { DI- } \\
\text { ELLRIN } \\
\text { TOTAL } \\
\text { (UG/L) }\end{array}$ & $\begin{array}{l}\text { ENDO- } \\
\text { SULFAN, } \\
\text { TOTAL } \\
\text { (UG/L) }\end{array}$ \\
\hline 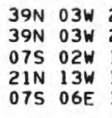 & 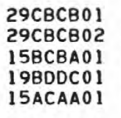 & $\begin{array}{l}\text { CHBYGN } \\
\text { CHBYGN } \\
\text { HILSDL } \\
\text { MANSTE } \\
\text { MONROE }\end{array}$ & $\begin{array}{l}79-08-23 \\
79-08-23 \\
79-08-20 \\
79-08-22 \\
79-08-09\end{array}$ & $\begin{array}{l}.00 \\
000 \\
000 \\
000 \\
-0\end{array}$ & $\begin{array}{l}.00 \\
000 \\
000 \\
0.00 \\
--\end{array}$ & $\begin{array}{l}.00 \\
000 \\
000 \\
000 \\
-0\end{array}$ & $\begin{array}{l}: 0 \\
: 0 \\
: 0 \\
: 0\end{array}$ & $\begin{array}{l}.00 \\
0.00 \\
0.00 \\
000 \\
-0\end{array}$ & $\begin{array}{l}.00 \\
000 \\
000 \\
000 \\
0 .-\end{array}$ & $\begin{array}{l}.00 \\
000 \\
000 \\
000 \\
0 .-\end{array}$ & $\begin{array}{l}.00 \\
000 \\
000 \\
.00 \\
-0\end{array}$ & $\begin{array}{l}.00 \\
.00 \\
0.00 \\
.00 \\
-.-\end{array}$ \\
\hline 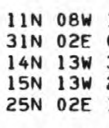 & 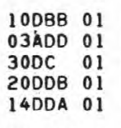 & $\begin{array}{l}\text { MNTCLM } \\
\text { MNTMRY } \\
\text { NEWYGO } \\
\text { NEWYGO } \\
\text { OSCODA }\end{array}$ & $\begin{array}{l}79-08-21 \\
79-08-23 \\
79-08-21 \\
79-08-21 \\
79-08-24\end{array}$ & $\begin{array}{l}.00 \\
000 \\
000 \\
00 \\
00\end{array}$ & $\begin{array}{l}.00 \\
000 \\
000 \\
000 \\
.00\end{array}$ & $\begin{array}{l}.00 \\
000 \\
00 \\
00 \\
000 \\
.00\end{array}$ & $\begin{array}{l}.0 \\
00 \\
00 \\
.0 \\
0\end{array}$ & $\begin{array}{l}.00 \\
.00 \\
0.00 \\
.00 \\
.00\end{array}$ & $\begin{array}{l}.00 \\
.00 \\
00 \\
000 \\
.00 \\
.00\end{array}$ & $\begin{array}{l}.00 \\
.00 \\
00 \\
00 \\
.00 \\
.00\end{array}$ & $\begin{array}{l}.00 \\
.00 \\
00 \\
00 \\
.00 \\
.00\end{array}$ & $\begin{array}{l}.00 \\
.00 \\
.00 \\
.00 \\
.00\end{array}$ \\
\hline $\begin{array}{l}30 \mathrm{~N} \\
03 \mathrm{~W} \\
34 \mathrm{~N} \\
227 \mathrm{~N} \\
22 \mathrm{~N}\end{array}$ & 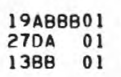 & $\begin{array}{l}\text { OTSEGO } \\
\text { PRSQ I } \\
\text { WEXFRD }\end{array}$ & $\begin{array}{l}79-08-23 \\
79-08-22\end{array}$ & $\begin{array}{l}.00 \\
000 \\
.00\end{array}$ & $\begin{array}{l}.00 \\
.00\end{array}$ & $\begin{array}{l}00 \\
.00\end{array}$ & $\stackrel{0}{0} 0$ & $\begin{array}{l}.00 \\
.00 \\
.00\end{array}$ & $\begin{array}{l}.00 \\
.00 \\
.00 \\
.00\end{array}$ & $\begin{array}{l}.00 \\
.00 \\
.00\end{array}$ & $\begin{array}{l}.00 \\
: 00 \\
.00\end{array}$ & $\begin{array}{l}.00 \\
.00 \\
.00\end{array}$ \\
\hline
\end{tabular}

\section{LOCAL
IDENT-
I-
FIER}

$39 \mathrm{~N} 03 W 29 \mathrm{CBCBO} 01$ CHBYGN $39 \mathrm{~N} 03 W$ 29CBCB02 CHBYGN 075 02W 15BCBA01 HILSOL 2IN 13W 19BDDCOI MANSTE O7S O6E ISACAAOI MONROE

$11 \mathrm{~N} 08 \mathrm{~W}$ 100BB 01 MNTCLM 3IN O2E O3ADD 01 MNTMRY $14 \mathrm{~N} 13 \mathrm{~W} 300 \mathrm{C}$ OI NEWYGO $15 \mathrm{~N} 13 \mathrm{~W} 20 \mathrm{DDB}$ OI NEWYGO $25 \mathrm{~N}$ O2E I4DOA OI OSCODA

$30 \mathrm{~N} \mathrm{03W} \mathrm{19ABBBOI}$ OTSEGO $34 \mathrm{~N}$ O7E $270 A$ OI PRSQ I $22 \mathrm{~N} 12 \mathrm{~W} 13 \mathrm{BB}$ OI WEXFRD

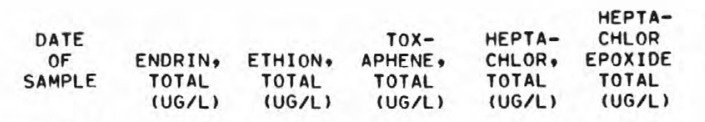

METH-
OXY-
CHLOR,
TOTAL PCB,
TOTAL
(UG/L)

MALA- PARA-

(UG/L)

.00
.00
.00
.00
.-0

.00
.00
.00
.00

$79-08-23$
$79-08-5$

$79-08-25$

$79-08-22$
$79-08-09$

.00
.00
.00
.00

$79-08-21$

79-08-23

$79-08-21$

$79-08-21$

79-08-24

.00

.00
.00

.00
.00

.00
.00

.00

.00

.0
.0
.0
.0

(UG/L)

THION,

TOTAL
(UG/L)

$79-08-23$

$79-08-23$

$79-08-22$
.00

.00

.00

.00
.00

.00
.00

.00

$.00 \quad .00$

$\begin{array}{ll}.00 & .00 \\ .00 & .00\end{array}$

$\begin{array}{ll}.00 & .00 \\ .00 & .00 \\ .00 & .00 \\ .00 & .00 \\ .00 & .00 \\ .00 & .00 \\ .00 & .00 \\ .00 & .00 \\ .00 & .00 \\ .00 & .00 \\ .00 & .00 \\ .00 & .00 \\ .00 & .00\end{array}$


TABLE 3. WATER-QUALITY DATA. (CONTINUED)

\begin{tabular}{|c|c|c|c|c|c|c|c|c|c|}
\hline & & $\begin{array}{l}\text { LOCAL } \\
\text { IDENT- } \\
\text { I- } \\
\text { FIER }\end{array}$ & & $\begin{array}{c}\text { DATE } \\
\text { OF } \\
\text { SAMPLE }\end{array}$ & $\begin{array}{l}\text { DI- } \\
\text { AZINON, } \\
\text { TOTAL } \\
\text { (UG/L) }\end{array}$ & $\begin{array}{l}\text { METHYL } \\
\text { PARA- } \\
\text { THION, } \\
\text { TOTAL } \\
\text { (UG/L) }\end{array}$ & $\begin{array}{l}2,4-0, \\
\text { TOTAL } \\
\text { (UG/L) }\end{array}$ & $\begin{array}{l}2,4,5-T \\
\text { TOTAL } \\
\text { (UGLL) }\end{array}$ & $\begin{array}{l}\text { MIREX, } \\
\text { TOTAL } \\
\text { (UG/L) }\end{array}$ \\
\hline $\begin{array}{l}39 \mathrm{~N} \\
39 \mathrm{~N} \\
07 \mathrm{~S} \\
21 \mathrm{~N} \\
07 \mathrm{~S}\end{array}$ & $\begin{array}{l}03 W \\
03 W \\
02 W \\
13 W \\
06 E\end{array}$ & $\begin{array}{l}29 \text { СВCB } 01 \\
29 \text { CBCB02 } \\
15 B C B A 01 \\
198 D D C 01 \\
15 A C A A 01\end{array}$ & $\begin{array}{l}\text { CHBYGN } \\
\text { CHBYGN } \\
\text { HILSDL } \\
\text { MANSTE } \\
\text { MONROE }\end{array}$ & $\begin{array}{l}79-08-23 \\
79-08-23 \\
79-08-20 \\
79-08-22 \\
79-08-09\end{array}$ & $\begin{array}{l}.00 \\
.00 \\
.00 \\
.00 \\
.01\end{array}$ & $\begin{array}{l}.00 \\
.00 \\
.00 \\
.00 \\
.00\end{array}$ & $\begin{array}{l}.00 \\
.00 \\
.00 \\
-0 \\
.00\end{array}$ & $\begin{array}{l}.00 \\
.00 \\
.00 \\
.00\end{array}$ & $\begin{array}{l}.00 \\
.00 \\
.00 \\
.00 \\
.00\end{array}$ \\
\hline $\begin{array}{l}11 \mathrm{~N} \\
31 \mathrm{~N} \\
14 \mathrm{~N} \\
15 \mathrm{~N} \\
25 \mathrm{~N}\end{array}$ & $\begin{array}{l}08 \mathrm{~W} \\
02 E \\
13 \mathrm{~W} \\
13 \mathrm{~W} \\
02 E\end{array}$ & $\begin{array}{ll}100 B B & 01 \\
03 A D D & 01 \\
30 D C & 01 \\
20 D D B & 01 \\
140 D A & 01\end{array}$ & $\begin{array}{l}\text { MNTCLM } \\
\text { MNTMRY } \\
\text { NEWYGO } \\
\text { NEWYGO } \\
\text { OSCODA }\end{array}$ & $\begin{array}{l}79-08-21 \\
79-08-23 \\
79-08-21 \\
79-08-21 \\
79-08-24\end{array}$ & $\begin{array}{l}.00 \\
.00 \\
.00 \\
.00 \\
.00\end{array}$ & $\begin{array}{l}.00 \\
.00 \\
.00 \\
.00 \\
.00\end{array}$ & $\begin{array}{l}.00 \\
.00 \\
.00 \\
.0 \\
.00\end{array}$ & $\begin{array}{l}.00 \\
.00 \\
.00 \\
.0 \\
.00\end{array}$ & $\begin{array}{l}.00 \\
.00 \\
.00 \\
.00 \\
.00\end{array}$ \\
\hline $\begin{array}{l}30 \mathrm{~N} \\
34 \mathrm{~N} \\
22 \mathrm{~N}\end{array}$ & $\begin{array}{l}03 \mathrm{~W} \\
07 \mathrm{E} \\
12 \mathrm{~W}\end{array}$ & $\begin{array}{ll}19 A B B B & 1 \\
270 A & 01 \\
13 B B & 01\end{array}$ & $\begin{array}{l}\text { OTSEGO } \\
\text { PRSO I } \\
\text { WEXFRD }\end{array}$ & $\begin{array}{l}79-08-23 \\
79-08-23 \\
79-08-22\end{array}$ & $\begin{array}{l}.00 \\
.00 \\
.00\end{array}$ & $\begin{array}{l}.00 \\
.00 \\
.00\end{array}$ & $\begin{array}{l}.10 \\
.00 \\
.00\end{array}$ & $\begin{array}{l}.00 \\
.00 \\
.00\end{array}$ & $\begin{array}{l}.00 \\
.00 \\
.00\end{array}$ \\
\hline & & $\begin{array}{l}\text { LOCAL } \\
\text { IDENT- } \\
\text { I- } \\
\text { FIER }\end{array}$ & & $\begin{array}{c}\text { DATE } \\
\text { OF } \\
\text { SAMPLE }\end{array}$ & $\begin{array}{l}\text { SOLIDS, } \\
\text { SUM OF } \\
\text { CONSTI- } \\
\text { TUENTS, } \\
\text { DIS- } \\
\text { SOLVED } \\
\text { (MG/L) }\end{array}$ & $\begin{array}{l}\text { PHOS- } \\
\text { PHORUS, } \\
\text { ORTHOPH } \\
\text { OSPHATE } \\
\text { TOTAL } \\
\text { (MG/L } \\
\text { AS P) }\end{array}$ & $\begin{array}{l}\text { NITRO- } \\
\text { GEN, } \\
\text { TOTAL } \\
\text { (MG/L } \\
\text { AS NO3) }\end{array}$ & $\begin{array}{l}\text { MERCURY } \\
\text { TOTAL } \\
\text { RECOV- } \\
\text { ERABLE } \\
\text { (UG/L } \\
\text { AS HG) }\end{array}$ & $\begin{array}{l}\text { URANIUM } \\
\text { DIS- } \\
\text { SOLVED, } \\
\text { EXTRAC- } \\
\text { TION } \\
\text { (UG/L) }\end{array}$ \\
\hline $\begin{array}{l}39 \mathrm{~N} \\
39 \mathrm{~N} \\
07 \mathrm{~S} \\
21 \mathrm{~N} \\
07 \mathrm{~S}\end{array}$ & $\begin{array}{l}03 W \\
03 W \\
02 W \\
13 W \\
06 E\end{array}$ & $\begin{array}{l}\text { 29СВСВ01 } \\
29 \text { СССB } 02 \\
15 B C B A 01 \\
19 B D D C 01 \\
15 A C A A 01\end{array}$ & $\begin{array}{l}\text { CHBYGN } \\
\text { CHBYGN } \\
\text { HILSOL } \\
\text { MANSTE } \\
\text { MONROE }\end{array}$ & $\begin{array}{l}79-08-23 \\
79-08-23 \\
79-08-20 \\
79-08-22 \\
79-08-09\end{array}$ & $\begin{array}{l}159 \\
155 \\
352 \\
140 \\
253\end{array}$ & $\begin{array}{l}.00 \\
.00 \\
.00 \\
.00 \\
.00\end{array}$ & $\begin{array}{l}2.1 \\
2.0 \\
1.3 \\
1.2 \\
21\end{array}$ & $\begin{array}{l}<.5 \\
<.5 \\
<.5 \\
<.5 \\
<.5\end{array}$ & $\begin{array}{l}.33 \\
.04 \\
.62 \\
.09 \\
.10\end{array}$ \\
\hline $\begin{array}{l}11 \mathrm{~N} \\
31 \mathrm{~N} \\
14 \mathrm{~N} \\
15 \mathrm{~N} \\
25 \mathrm{~N}\end{array}$ & $\begin{array}{l}08 W \\
02 E \\
13 W \\
13 W \\
02 E\end{array}$ & $\begin{array}{ll}100 B B & 01 \\
03 A D D & 01 \\
30 D C & 01 \\
20 D D B & 01 \\
14 D D A & 01\end{array}$ & $\begin{array}{l}\text { MNTCLM } \\
\text { MNTMRY } \\
\text { NEWYGO } \\
\text { NEWYGO } \\
\text { OSCODA }\end{array}$ & $\begin{array}{l}79-08-21 \\
79-08-23 \\
79-08-21 \\
79-08-21 \\
79-08-24\end{array}$ & $\begin{array}{r}253 \\
234 \\
195 \\
73 \\
198\end{array}$ & $\begin{array}{l}.00 \\
.00 \\
.01 \\
.00 \\
.00\end{array}$ & $\begin{array}{l}3.8 \\
.53 \\
4.9 \\
4.4 \\
13\end{array}$ & $\begin{array}{l}<.5 \\
<.5 \\
<.5 \\
<.5 \\
<.5\end{array}$ & $\begin{array}{r}.30 \\
.74 \\
<.01 \\
.11 \\
.23\end{array}$ \\
\hline $\begin{array}{l}30 \mathrm{~N} \\
34 \mathrm{~N} \\
22 \mathrm{~N}\end{array}$ & $\begin{array}{l}03 \mathrm{~W} \\
07 \mathrm{E} \\
12 \mathrm{~W}\end{array}$ & $\begin{array}{ll}\text { 19ABBB } 01 \\
270 A & 01 \\
13 B B & 01\end{array}$ & $\begin{array}{l}\text { OTSEGO } \\
\text { PRSQ I } \\
\text { WEXFRD }\end{array}$ & $\begin{array}{l}79-08-23 \\
79-08-23 \\
79-08-22\end{array}$ & $\begin{array}{l}217 \\
642 \\
169\end{array}$ & $\begin{array}{l}.00 \\
.00 \\
.01\end{array}$ & $\begin{array}{l}2.2 \\
1.1 \\
.04\end{array}$ & $\begin{array}{l}<.5 \\
<.5 \\
<.5\end{array}$ & $\begin{array}{l}.16 \\
.29 \\
.48\end{array}$ \\
\hline
\end{tabular}




\section{Publications of the U.S. Geological Survey}

Water-Supply Papers

Allen, W. B., Miller, J. B., and Wood, W. W., 1972, Availability of water in Kalamazoo County, Michigan: U.S. Geological Survey Water-Supply Paper 1973, 129 p., 36 figs., 9 pls.

Deutsch, Morris, 1963, Ground-water contamination and legal controls in Michigan: U.S. Geological Survey Water-Supply Paper 1691, 79 p., 23 figs.

Lohr, E. W., and Love, S. K., 1954, The industrial utility of public water supplies in the United States 1952, pt. 1, States east of the Mississippi River: U.S. Geological Survey Water-Supply Paper 1299, 639 p., 3 figs., 5 pls.

McGuinness, C. L., 1963, The role of ground water in the national water situation: U.S. Geologica1 Survey Water-Supply Paper 1800, 1121 p., 2 figs., 4 pls.

McGuinness, C. L., Poindexter, O. F., and Otten, E. G., 1949, Ground-water supplies of the Ypsilanti area, Michigan: U.S. Geological Survey Water-Supply Paper 1078, 105 p., 7 figs., 5 pls.

Reed, J. E., Deutsch, Morris, and Wiitala, S. W., 1966, Induced recharge of an artesian glacial-drift aquifer at Kalamazoo, Michigan: U.S. Geological Survey Water-Supply Paper 1594-D, 62 p., 36 figs., 2 pls.

Twenter, F. R., and Knutilla, R. L., 1972, Water for a rapidly growing urban community--Oakland County, Michigan: U.S. Geological Survey Water-Supply Paper 2000, 150 p., 90 figs.

Vanlier, K. E., 1963, Ground-water resources of the Alma area, Michigan:

U.S. Geological Survey Water-Supply Paper 1619-E, 66 p., 20 figs., 2 pls.

Vanlier, K. E., Wood, W. W., and Brunett, J. O., 1973, Water-supply development and management alternatives for Clinton, Eaton, and Ingham Counties, Michigan: U.S. Geological Survey Water-Supply Paper 1969, 111 p., 35 figs., 3 pls.

Wiitala, S. W., Newport, T. G., and Skinner, E. L., 1967, Water resources of the Marquette Iron Range area, Michigan: U.S. Geologica1 Survey WaterSupply Paper 1842, 142 p., 40 figs., 4 pls.

Wiitala, S. W., Vanlier, K. E., and Krieger, R. A., 1963, Water resources of the Flint area, Michigan: U.S. Geological Survey Water-Supply Paper 1499-E, 86 p., 32 figs,, 6 pls. 
Water-Supply Papers (contain ground-water data for Michigan)

\begin{tabular}{|c|c|c|c|c|c|}
\hline Year & $\begin{array}{c}\text { WSP } \\
\text { Number }\end{array}$ & Year & $\begin{array}{l}\text { WSP } \\
\text { Number }\end{array}$ & Year & $\begin{array}{l}\text { WSP } \\
\text { Number }\end{array}$ \\
\hline 1935 & 777 & 1944 & 1016 & 1953 & 1265 \\
\hline 1936 & 817 & 1945 & 1023 & 1954 & 1321 \\
\hline 1937 & 840 & 1946 & 1071 & 1955 & 1404 \\
\hline 1938 & 845 & 1947 & 1096 & $1956-57$ & 1537 \\
\hline 1939 & 886 & 1948 & 1126 & $1958-62$ & 1782 \\
\hline 1940 & 906 & 1949 & 1156 & $1963-67$ & 1977 \\
\hline 1941 & 936 & 1950 & 1165 & $1968-72$ & 2140 \\
\hline 1942 & 944 & 1951 & 1191 & & \\
\hline 1943 & 986 & 1952 & 1221 & & \\
\hline
\end{tabular}

\section{Circulars}

Murray, C. R., and Reeves, E. B., 1977, Estimated use of water in the United States, 1975: U.S. Geological Survey Circular 765, 39 p., 12 figs.

Strame1, G. J., Wisler, C. O., and Laird, L. B., 1954, Water resources of the Grand Rapids area, Michigan: U.S. Geological Survey Circular 323, 40 p., 29 figs., 3 pls.

Wisler, C. O., Stramel, G. J., and Laird, L. B., 1952, Water resources of the Detroit area, Michigan: U.S. Geological Survey Circular 183, 36 p., 30 figs., 4 pls.

Water-Data Reports

\begin{tabular}{|c|c|}
\hline Year & WDR Number \\
\hline 1975 & MI-75-1 \\
\hline 197 & MI- $76-1$ \\
\hline 19 & MI - 77-1 \\
\hline 197 & MI- $78-1$ \\
\hline 19 & MI - 79-1 \\
\hline
\end{tabular}

\section{Other Publications}

Allen, W. B., 1977, Flowing wells in Michigan, 1974: Michigan Geological Survey Water Information Series Report 2, 27 p., 5 figs., 2 pls., 16 refs.

Borton, T. E., 1974, Planning perspectives on water resources, Washtenaw County, Michigan: Washtenaw County Metropolitan Planning Commission, $69 \mathrm{p}$. 
Brown, E. A., and Stuart, W. T., 1951, Ground-water resources of the glacial deposits in the Bessemer area, Michigan, 1950: Michigan Geologica1 Survey Progress Report 14, 68 p., 27 figs.

Deutsch, Morris, 1956, Effects of dissemination of radioactive materials on water resources conservation--with special references to Michigan: Michigan State University Agricultural Experiment Station Water Bulletin 2.

--- 196la, Hydrogeologic aspects of ground-water pollution: Water Well Journal, v. 15, no. 9.

--.- 1961b, Incidents of chromium contamination of ground-water in Michigan: U.S. Public Health Service Technical Report W61-5, p. 98-104.

-..- 1962a, Controlled induced-recharge tests at Kalamazoo, Michigan: Journal of American Water Works Association, v. 54, no. 2, p. 181-196.

---- 1962b, Phenol contamination of an artesian aquifer at Alma, Michigan: Proceedings of the Society for Water Treatment and Examination, v. 11, p. 94-100, 2 figs.

Deutsch, Morris, Burt, E. M., and Van1ier, K. E., 1958, Summary of groundwater investigations in the Holland area, Michigan: Michigan Geological Survey Progress Report 20, 87 p., 16 figs.

Deutsch, Morris, Vanlier, K. E., and Giroux, P. R., 1960, Ground-water hydrology and glacial geology of the Kalamazoo area, Michigan: Michigan Geological Survey Progress Report 23, 122 p., 21 figs.

Deutsch, Morris, and Vanlier, K. E., 1961, Ground water for Michigan's future: U.S. Geological Survey open-file report.

Doonan, C. J., and Byerlay, J. R., 1973, Ground water and geology of Baraga County, Michigan: Michigan Geological Survey Water Investigation 11, 26 p., 2 figs., 2 pls.

Doonan, C. J., and Hendrickson, G. E., 1967, Ground water in Iron County, Michigan: Michigan Geological Survey Water Investigation 7, 61 p., 3 figs., 2 pls.

1968a, Ground water in Gogebic County, Michigan: Michigan Geological Survey Water Investigation 8, 22 p., 5 figs., 2 pls.

---- 1969, Ground water in Ontonagon County, Michigan: Michigan Geologica1 Survey Water Investigation 9, 29 p., 5 figs., 1 pl.

Doonan, C. J., Hendrickson, G. E., and Byerlay, J. R., 1970, Ground water and geology of Keweenaw Peninsula, Michigan: Michigan Geological Survey Water Investigation 10, 41 p., 1 fig., 2 pls. 
Ferris, J. G., and others, 1954, Ground-water resources of southeastern Oakland County, Michigan: Michigan Geological Survey Progress Report 16, 158 p., 44 figs., 6 pls.

Fleck, W. B., 1980, Geology and hydrology for enviromental planning in Washtenaw County, Michigan: U.S. Geological Survey Open-File Report unmumbered, 23 p., 21 figs., 14 recs.

Fleck, W. B., and McDonald, M. G., 1978, Three-dimensional finite-difference model of ground-water systems underlying the Muskegon County wastewater disposal system, Michigan: U.S. Geological Survey Journal of Research, v. 6, no. 3, p. 307-318, 16 figs., 14 refs.

Giroux, P. R., Hendrickson, G. E., Stoimenoff, L. E., and Whetstone, G. W., 1964, Water resources of Van Buren County, Michigan: Michigan Geological Survey Water Investigation 3, 144 p., 45 figs.

Giroux, P. R., Stoimenoff, L. E., Now1in, J. O., and Skinner, E. L., 1966, Water resources of Branch County, Michigan: Michigan Geological Survey Water Investigation 6, 158 p., 34 figs., 2 pls.

Grannemann, N. G., 1978, Water Supply Potential of the Lake Sally System, Marquette County, Michigan: U.S. Geological Survey Open-File report 78-1046, 14p., 6 figs., 1 table, 12 refs.

Grannemann, N. G., 1979, Water Resources of the Marquette Iron Range area, Marquette County, Michigan: U.S. Geological Survey Open-File report 79-1339, 77 p., 34 figs., 20 tables, 23 refs.

Great Lakes Basin Commission, 1975, Appendix 3, Geology and ground water: Great Lakes Basin Framework Study, 152 p., 15 tables, 60 figs.

Hendrickson, G. E., 1966, Michigan's Au Sable River--Today and tomorrow: Michigan Geological Survey Bulletin 3, 80 p., 29 figs., 11 photos.

Hendrickson, G. E., and Doonan, C. J., 1966, Ground-water resources of Dickinson County, Michigan: Michigan Geological Survey Water Investigation 5, 49 p., 5 figs., 3 pls.

Huffman, G. C., 1979, Ground-water data for Michigan, 1977, U.S. Geologica1 Survey Open-File report 79-332, 75 p., 5 figs.

Knutilla, R. L., Twenter, F. R., and Larson, R. W., 1971, Upper Rifle River Basin--An evaluation of its water resources and hydrologic environment: Michigan Geological Survey Water Information Series Report 1, 66 p., 64 figs.

McDonald, M. G., and Fleck, W. B., 1978, Model analysis of the impact on ground-water conditions of the Muskegon County wastewater disposal system, Michigan: U.S. Geological Survey Open-File Report 78-99, 63 p., 17 figs., 14 refs.

Michigan Department of Health, 1961, Data on public water supplies in Michigan: Michigan Department Health Engineering Bulletin 4, 57 p. 
Mozola, A. J., 1954, A survey of ground-water resources in Oakland County, Michigan, pt. 2 of Occasional papers for 1954 on the geology of Michigan: Michigan Geological Survey Publication 48, p. 96-348, 31 figs.

-.-- 1969, Geology for 1and and ground-water development in Wayne County, Michigan: Michigan Geological Survey Report Investigation 3, 25 p., 9 figs., 4 pls.

---- 1970, Geology for environmental planning in Monroe County, Michigan: Michigan Geological Survey Report Investigation 13, 34 p., 18 figs., 6 pls.

Sinclair, W. C., 1959, Reconnaissance of the ground-water resources of Schoolcraft County, Michigan: Michigan Geological Survey Progress Report 22, 84 p., 14 figs.

Sinclair, W. C., 1960, Reconnaissance of the ground-water resources of Delta County, Michigan: Michigan Geological Survey Progress Report 24, 93 p., 13 figs.

Stuart, W. T., 1945, Ground-water resources of the Lansing area, Michigan: Michigan Geological Survey Progress Report 13, 35 p., 11 figs.

Stuart, W. T., Brown, E. A., and Rhodehamel, E. C., 1954, Ground-water investigations of the Marquette iron-mining district, Michigan: Michigan Geological Survey Technical Report 3, 92 p., 8 figs.

Stuart, W. T., and Stallman, R. W., 1945, Ground-water resources of the Benton Harbor area, Michigan: Michigan Geological Survey Progress Report 12, 15 p., 4 figs.

Stuart, W. T., Theis, C. V., and Stanley, G. M., 1948, Ground-water problems in the Iron River district, Michigan: Michigan Geological Survey Technical Report 2, 59 p., 16 figs.

Terwilliger, F. W., 1954, The glacial geology and ground-water resources of Van Buren County, Michigan pt. I of Occasional papers for 1954

on the geology of Michigan: Michigan Geological Survey Publication 48, p. 1-95, 24 figs., 1 pl.

Twenter, F. R., 1975, Ground water and geology--Southeastern Michigan: U.S. Army Corps of Engineers, Detroit, Michigan, 143 p., 31 figs., 36 tables, 46 refs.

Twenter, F. R., Knutilla, R. L., and Nowlin, J. O., 1976, Water resources of Washtenaw County, Michigan: Washtenaw County Planning Commission, 143 p., 43 figs., 18 tables, 25 refs.

Vanlier, K. E., 1959, Reconnaissance of the ground-water resources of Luce County, Michigan: Michigan Geological Survey Progress Report 21, 76 p., 11 figs., 3 p1s. 
Vanlier, K. E., 1962, Summary of ground-water investigations in the E1sie area, Michigan: Michigan Geological Survey Progress Report 25, 35 p., 7 figs.

-.-- 1963a, Ground water in A1ger County, Michigan: Michigan Geologica1 Survey Water Investigation 1, 55 p., 13 figs.

-.-- 1963b, Ground water in Menominee County, Michigan: Michigan Geological Survey Water Investigation 2, 42 p., 11 figs.

-..- 1966, Ground water resources of the Battle Creek area, Michigan: Michigan Geological Survey Water Investigation 4, 52 p., 19 figs.

---- 1968, Appendix E of the report on the Grand River Comprehensive Basin Study: U.S. Army Engineers District, Detroit, Michigan, 82 p., 2 figs.

Vanlier, K. E., and Deutsch, Morris, 1958a, Reconnaissance of the groundwater resources of Chippewa County, Michigan: Michigan Geological Survey Progress Report 17, 56 p., 7 figs., 7 pls.

1958b, Reconnaissance of the ground-water resources of Mackinac County, Michigan: Michigan Geological Survey Progress Report 19, 82 p., 8 figs., 6 pls.

Vanlier, K. E., and Wheeler, M. L., 1968, Analog simulation of ground-water development of the Saginaw Formation, Lansing Metropolitan area, Michigan: Tri-County Planning Commission, Lansing Ground-Water Report, 40 p., 23 figs.

\section{Michigan Water Resources Commission Reports}

Water resources of the Clinton River Basin, 1953.

Water resource conditions and uses in the Paw Paw River Basin, 1955 (revised report in 1964).

Water resources conditions and uses in the F1int River Basin, 1956. in the Huron River Basin, 1957. in the Tittabawassee River Basin, 1960. in the Upper Grand River Basin, 1961. in the Shiawassee River Basin, 1963. in the Maumee River Basin, 1964. in the River Raisin Basin, 1965. in the Au Sable River Basin, 1966. in the Lower Grand River Basin, 1967

(open file). 
Water resources of southeastern Michigan, February 1968.

Water resources of the lower Lake Huron drainage basin, May 1968.

Water quality standards for Michigan intrastate waters, January 1968.

Water quality standards for Michigan waters, Appendix A (interstate and internationa1 waters) June 1967.

Water resources uses, present and prospective, and water-quality standards and plan of implementation (revised June 1967) for . . .

Lake Superior and the St. Mary's River,

Lake Huron,

The Menominee and Montreal River basins in Michigan and the other Michigan-Wisconsin interstate boundary waters,

St. Clair River, Lake St. Clair, Detroit River, Lake Erie, and Maumee River basin,

Lake Michigan,

St. Joseph River basin.

Use designation areas for Michigan's intrastate water quality standards, March 1969.

Twenter, F. R., 1966a, General availability and quality of ground water in the bedrock deposits in Michigan: State Resources Planning Division, Michigan Department of Commerce and Michigan Water Resources Commission, map (color).

1966b, General availability of ground water in the glacial deposits in Michigan: State Resources Planning Division, Michigan Department of Commerce and Michigan Water Resources Commission, map (color).

\section{ACKNOWLEDGMENTS}

Acknowledgment is made to personnel of Federal and State agencies, county and township governments, industrial concerns, well drillers, consultants, municipalities, and public utilities, without whose cooperation the accumlation of data presented in this report would have not been possible.

The collection of ground-water data is aided by the following municipalities, institutions, and private organizations: 
Cities or villages of Ann Arbor, Battle Creek, Clare, Coldwater Jackson, Kalamazoo, Lansing, Marsha11, Mason, Portage, St. Johns, and Ypsilanti; Ypsilanti Township; counties of Branch, Van Buren, and Kalamazoo; U.S. Army Engineers; Kent Metropolitan Airport; HuronClinton Metropolitan Authority; Fisher Body Division of General Motors Corporation; Harman Internationa1 Industry; Brown Company; WisconsinMichigan Power Company; Cleveland-Cliffs Iron Company; UpJohn Company, and American Aggregates Corporation. 
